\title{
UC-NRLF
}

|||||||||||||||||||||||||||||||||||||||||||||||

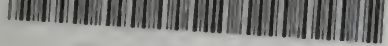

\section{B 4243595}

POLLEN

M. P. EDGEWORTH 


\section{Location}

Date and Number of Issue.
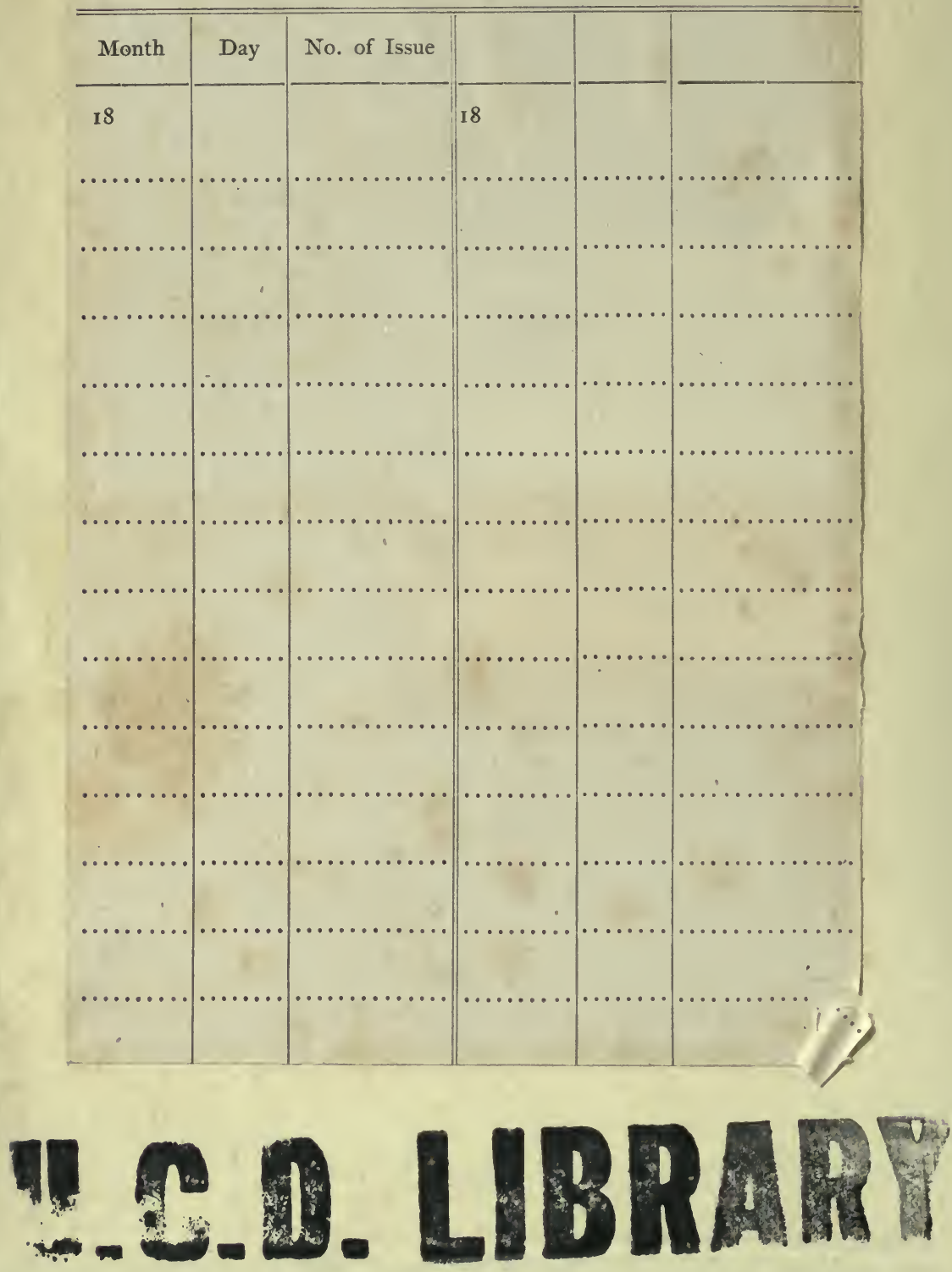
Digitized by the Internet Archive in 2007 with funding from Microsoft Corporation 



\section{P OLLEN}

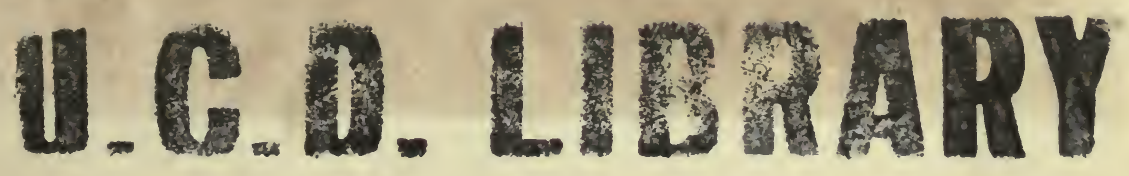


LONDON : PRINTED BY

SPOTTISWOODE AND CO., NEW-STREET SQUARE AXD PARLIAMENT STREET 


\section{P O L L E N}

BY

M. PAKENHAM EDGEWORTH, F.L.S. F.A.S.

ILLUSTRATED WITH' 438 FIGURES

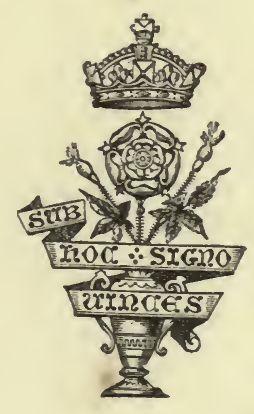

LONDON

HARDWICKE \& BOGUE, 192 PICCADILLY 


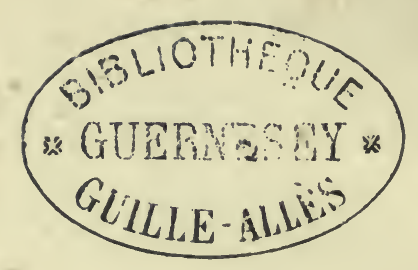




\section{P R E F A C E.}

LAST yEAR I laid before the Linnæan Society a Paper on Pollen, with drawings of the various kinds. But it having been brought to my notice that several German botanists had written on the subject of late years, and also that HASSALL had published an important paper on it, I withdrew mine from before the Society.

Since then I have altered it considerably; having added new matter, and omitted from the plates what had been figured previously by Hassali and Romanoff, except where I differed from them.

I also take notice of the drawings by Mr. WoRthington SмrтH, which were published by him after my paper had been laid before the Society; and have added to the list all that have been figured and noticed by former botanists on the subject; so that it may be viewed immediately in detail.

I trust that, thus altered, this paper may prove a not unimportant addition to our knowledge of the subject. 



\section{POLLEN.}

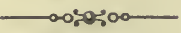

Two years ago Mr. Airey published some interesting facts about the dust collected on smeared plates of glass, and mentioned that some of the dust was pollen. I began accordingly to enquire into the forms of pollen likely to be found in such places, and from this went on to discover all that I could about pollen. I had many years ago drawn a number of specimens, but I now took to examining them more closely, and drawing them to scale.

The principal papers already published on this subject are by

1. Purjinke, in Latin. His illustrations of the cells of the anther are very interesting, but unfortunately, the pollens drawn have been treated with water, so as to lose most of their distinguishing marks.

2. By Mirbel, in French. Accurate as far as they go, but on too small a scale. ('Elémens de Physiologie végétale,' 1815.)

3. By Fritzsche, in German (1832). Most beautifully drawn both in the simple form and under oil, and under the effects of weak sulphuric acid. $\mathrm{He}$ examined almost all the natural families.

4. By Hugo Mohl, in German, translated into French in the 'Annales des Sciences Naturelles.' He goes most thoroughly through all the families in the natural state of the pollens, or as they appear under olive oil or water; drawn to scale. I have followed in his footsteps; and now I wish to point out a few inaccuracies into which he has fallen, and to carry on the examination of the other plants.

5. Hassall reviewed Mohl, showing some differences, and 
going through all the natural families in detail, with numerous plates drawn to scale, and published in the 'Annals of Nat. Hist.' 1842, vol. viii. ix.

6. Lindley published figures of pollen after some of the above authors; and

7. A further republication of them in the 'Micrographical Dictionary.'

8. Since that time Herman Schacht has published some beautiful drawings in the 'Jahrbuch der Botanik,' Berlin, 1860, ii. p. 109. I agree very much with what he has published, except about the Pinus family, of which his descriptions differ from what I have observed, and from the drawings of Mohl, and Hassall, and Dr. Hooker.

9. Nagilihas also a paper in the same publication, vol. iii. Rosanoff has a paper in the same publication (1865), vii. p. 34, principally on the pollen of the Acaciæ.

10. Leursen, in vol. vii. p. 34 of the same publication, reviews a paper by

11. M. Pollenden, Bonn, 1867 ;

12. A paper by Mr. A. W. Bennett in the 'Popular Science Review,' April, 1875 ; and lastly,

13. Some woodcuts by Mr. Worthington Smith in the 'Gardener's Chronicle' in October last; republished in the 'Microscopical Journal,' January, 1877.

The pollen forms are often noticed by Dr. Hooker in his large works, and also by some foreign botanists in the Brazil Flora.

It is remarkable, that while in some families the species vary greatly, in others they are almost always the same. Some may therefore be considered normal; others as having only a specific value. The different size of the grains is also marvellous: some very large, and visible to the naked eye, as in Stachytarpheta, the Malvaceæ, Cobæa, Pancratium, and some species of Iris, reaching as much as $\frac{1}{100}$ th of an inch; while others, as many Boragineæ, Primulaceæ, Melastomaceæ, and Saxifrageæ, are as small as $\frac{1 \frac{1}{2} \text { to } 2}{6000}$ ths of an inch. In colour also they are very various, but usually some shade of yellow or white; bat I have observed blue, red, brown, or rarely green, which has been denied by Lindley. 
Mr. Bennett would divide the pollen into those which are wind-borne, or anemophilous, and those which are not so scattered, or entomophilous; but the grains are by no means all globular, as he there asserts. The Gramineæ and Cyperaceæ are usually prismatic. The Populus has globular pollen, while the Salix, in the same family, is somewhat cylindric, as is that of Rheum, which is probably anemophilous, and the diœcious Rumices, with three slits.

Plantago, which appears to be also anemophilous, varies from a polyhedric to the simple form, while the wind-borne pollen of the Coniferæ is very different.

The Urticæ are very small and elliptic, with three slits, withering into a prismatic form.

The Grasses and Cyperaceæ, and perhaps the Plantagineæ, are without the sticky nature of the outer coat, which obtains through all other pollen grains.

Some grains throw out a quantity of tubes, by which they are hung together, as in Richardia agyptiaca, Strelitzia regina, and the Rhododendron, \&c., but more usually they are quite distinct. I omit any account of the peculiar pollen of the Asclepiadeæ and Orchideæ, which have not common loose grains of pollen, but joined together in a coherent mass. I also do not intend to make any remarks as to the structural nature and function of the fovilla and the pollen grains.

I have found but three or four instances in which the same anther produces two or three forms of pollen; usually they are all similar, often with some small or imperfect grains; but in the Mimulus luteus I have found the simple form, common in the Scrophulariaceæ, mixed with the coiled form, which $\mathrm{H}$. Mohl pointed out in the Mimulus moschatus. [ have not been able to find this coiled form myself in the latter, and Mr. W. Smith considers it an error of H. Mohl's; but in this he is doubtless mistaken, for $I$ have found it in the next species, M. luteus (No. 138). I have also found many forms of pollen in the Browallia elata (No. 146), in the same anther, but not in the coiled form; and H. Mohl mentions others also which I have not had the opportunities of examining.

I find also two forms in Thalictrum flavum and glaucum (Nos. 429-432), probably also in Ranunculus auricomus, as remarked by Hassall and myself in the Anisogeisos. 
The simplest form is a bag with one slit, from which the fovilla escapes, then opening out into a hat-shape, like many of the Liliaceæ and Amaryllideæ.

A similar form is seen in the Magnolia and Nuphar-a simple slit. It is curious that the simplest pollen should be found in one of the most ornate and largest of flowers!

This simple form is often found with ornate markings on the outside, in the Liliaceæ, for instance.

The next simplest form looks at first the same, but on examination it is found to contain three slits or three bands, the bands more or less broad. When floating in olive oil, so that all the sides of the grain are shown, the end is usually somewhat three-cornered, being the ends of the three slits or bands. This is universal, as far as I have seen, in the Saxifrageæ and Crassulaceæ, and almost universal in the Rosaceæ-(the Poterieæ (Nos. 286-7) and Spiræa palmata (No. 285) are the only exceptions I have seen. Hassall mentions another form with four slits in Rubus, but what I have examined had the usual simple form) - the Scrophulariacer, with the exception of Mimulus, which has three forms, and Browallia; which has many forms; most of the Solanaceæ, and all that I have examined of the Melastomacex, are similar, but are the smallest I have seen of this form. It is found in many other families, in which, however, there are also other forms; as in some Ranunculaceæ and Sapindaceæ, and in Spergularia rubra (No. 360) alone of the Caryophyllaceæ. The Primulaceæ have usually three slits, and when seen end up the pollen looks trigonous; but in some cases they have four or six slits, as remarked by Mr. A. W. Bennett in the 'Nat. Science Review' of 1875. P. cortusoides (No. 189 ) is both simple or trigonous, as I have examined, and as described by Mohl. Mohl and Hassall both describe the pollen of the Gramineæ as sub-globular, with one pore; but I have examined numerous specimens, and find it (with the exception of the Panicex, of which I have but seen two) always of one peculiar type-of a somewhat prismatic form, with rounded ends, larger at the top, and smaller at the bottom; while there are four sides with an oval or round opening in them, occasionally 3 -sided, 5 -sided, 6 -sided (No. 104). Mirbel describes the Phleum as I have observed it. Mr. A. W. Bennett describes this form in Briza, but says the Dactylis is 
oval; but I think he cannot have floated it in oil, or he would have seen the same form as in Briza. I fancy that Mohl and Hassall chanced merely to see the upper side.

Arundinaria, Lagurus, and Pogonatherum have a 6-sided prism, as represented by Mr. W. Smith. The only Panicum I have examined is $P$. plicatum (No. 5), which is quite different from any of the other Gramineæ, being globular with three pores; and there is something of the same kind in Streptium, a S. American genus of the same sub-order. The Cyperaceæ have two forms-one like a drop of water (in Cladium and Scirpus); in Carex it is somewhat prismatic, with a pore in each facet, which is the form of the Juncaceæ. Drimopsis in that family is peculiar, like a tooth, a deformed crystal of the Carex type (No. 23).

To return to the more banded forms. The Papilionaceæ have generally three broad bands; when seen endwise the pollen looks trigonous (the Erythrina and Indigofera have really a trigonous form, not cylindrical). Some are broader than others, but of a similar type, which obtains also more or less among the Cæsalpinieæ, with the exception of the Bauhinia, which is of a very different type, large and spherical, and echinulate, or large and trigonous. The Acacieæ are (with the exception of Mimosa pudica and in a few species) composed of nine to twelve or sixteen cells, in squares or pyramids, or cubical arrangement. The Cruciferæ have also three bands, smooth or rough, usually containing much oil, which is diffused in the olive oil. Schizopetalus is rougher, very richly marked, with no distinct slits (No. 385). Mr. Bennett has remarked the globular form of Pringlia, but it is possible this form has been assumed after being examined under water, or having been preserved in spirit. The Apocynaceæ, Jasmineæ, and Rutaceæ, in general are 3-banded, often with rich ornamentation on the outer coat, and containing much oil, which is diffused in olive oil, as is also the case with the very oily Tiliaceæ. Adenandra fragans, in Rutaceæ (No. $315)$ has two forms, one slightly curved, the other having an additional coat across the lower one: this is also found in the Umbelliferæ, which have also three slits, but are generally constricted in the centre. There are several forms of trigonous pollen. The Myrtaceæ, as far as I have observed, are all the same; the Proteaceæ (except Banksia, which has 
two instead of three pores at each end); many of the Verbenaceæ, as Verbena (No. 105) and Lippia; while Stachytarpheta is of large size $\left(\frac{1}{120} \mathrm{th}\right.$ of an inch), with its three pores, containing large craters, figured by Fritzsche and Hassall.

The Hydrophyllaceæ, and most of the Cucurbitaceæ, finally, the very large Onagraceæ, which are well known and described, also are trigonous, but in that family some of the Fuchsias ( $F$.thymifolium) have but two pores, like Banksia; and Epilobium roseum is compound, as described by Hassall. The Dianella, Stypandra in Aspariginea, are also trigonous, but in a very different nature, having a single cell with the slit, so as to make a cocked-hat form, not with a pore in each facet, as is the case in true trigonous pollen.

Many have polyhedric pollens, each part having a pore, sometimes smooth, sometimes echinate, as many Polygona, the Caryophyllaceæ, Amaranthaceæ, Chenopodiaceæ, and Alisma (No. 31). Many are globular; some simple, as Canna (No. 46), Costus, Musa (No. 45), Strelitzia; some smooth, some echinate, as Crocus* (No. 38), and Philesia (No. 15), which are richly echinate, and the Potomagetons. Some of the Nymphaceæ are echinate, as Nelumbium (Nos. 400-403) and the British species of Nymphæa, but the foreign are simply 3-banded (Nos. 401-2). The Passifloreæ, with large pores, are beautifully portrayed by Fritzsche. The most ornate are among the Synanthereæ, most of which are polyhedric or echinate, oval or globular, with three or more pores in them; a few are 3-slitted, as Artemisia (No. 213), and some Centaureæ.(No. 218), and the Mutisaceæ (No. 209).

The Malvaceæ have large pollen, and many of the Convolvulaceæ; Cobæa and Mirabilis are among the largest I know, and Cucurbita is one of the most beautiful (figured by Fritzsche and Hassall and Pollenden, and well drawn by Bauer, in the British Museum).

Some of the Clerodendrons are globular, others nearly square. Some of the Caprifolaciaceæ and Rubiaceæ are globular; but they have many other forms, some large, some

* In the genus Crocus, which always is globular, some species are quite smooth and shining, others more or less echinulate; while in $C$. masiacus, according to Mohl, and in $C$. nivalis, the outer coat is spiral; but it disappears in water, the extine being thus invisible. 
very minute. Diphylleia in the Berberideæ is echinate, while the greater part of that family is smooth and globular; others (Epimedium) have three bands; many others in the Malpighiaceæ are globular, with pores on the equator, or variously disposed.

Similarly in the large genus Ranunculus. $R$. arvensis (No. 425) alone is echinate; most of that species have three bands, while many of the Anemone have also three slits or bands; A. coronaria (No. 424) is dodecahedric, and A. pavonina (No. 425 ) is dodecahedric, and has rough globes, not however echinate. Many of the Dipsacaceæ are also sub-globular, but some have fantastic forms: the Morina is like a spigot (given by Mohl, and observed by myself in Indian specimens).

Some of the true Campanulaceæ have pollen covered with small echinations, which disappear in oil; others are smooth, while the Lobeliaceæ are all small, with three slits. In the sub-order of Epacrideæ, Styphileæ is echinate, while the true Epacris has compound pollen like the Ericaceæ. The true Geranieæ sub-order is large, rough, or sub-echinate, and the Viviana is similar, but smaller. The Tropæolum has usually three bands, which assume a trigonous form when seen endwise, as figured by Pollenden; but one species, Tropceolum tricolor, is very different (No. 327), a curved narrow form, not very far from the peculiar crescent shape of Limnanthes (Nos. 329, 330); another species of Limnanthes is figured by Hassall, somewhat sinilar, but echinate. Balsamineæ are somewhat oblong, with three bands or little pores, and the Oxalideæ are usually globular or oblong, smooth or rough.

The Boragineæ generally have the pollen in the form of a dumb-bell, but some are of the more common 3-slitted form, or oval. The Polygaleæ have a peculiar form; in the natural state oval with narrow bands. When made more visible by olive oil they show a beautiful form like a wickerwork basket, varying slightly in the different species. Some of the other genera are not similar, but of a simple form (Nos. 367, 368, 369, 380).

The Papaveraceæ have many forms, some simple, some globular; some of the Fumariaceæ are very peculiar, cubical, with large pores (Fumuria officinalis), figured by Hassall.

The Violaceæ, as remarked by Mr. Bennett, are penta- 
gonal, having five bands (four in Hassall's drawing); while V.odora (No. 375) and cornuta (No. 376) have three small slits. A garden hybrid between $V$. tricolor (No. 370) and $V$. cornuta, called Perfection (No. 377), shows some of the characteristics of both parents. This is very remarkable, as Mr. Worthington Smith has pointed out that it is impossible to cross species which have different pollens; but this does not seem to be the case at any rate. No doubt the subject will be carefully examined by hybridists, to whom it is most important. The Pinus and Abies (No. 37) have a very peculiar form; two grains of pollen connected as it were by a broad band. This is distinctly shown by Mohl or Hassall, and confirmed by my own observations. I mention this as Schacht describes them differently, and I cannot but believe him mistaken. Dr. Hooker describes some in his Tasmanian Flora somewhat similar, but with three instead of two grains of pollen.

Other Gymnosperms are quite different, sub-globular, with four coats; in water or acid they burst at one side with a large opening. Some are quite simple, as Ephedra, or the Cycadeæ (No. 47).

We have, further, several compound pollens, three or four cells united into one, as in Ericaceæ (with the exception of Clethra, which has the common 3-slit form), Epacris, Randia longiflora, and a few other Rubiaceæ. Epilobium roseum in Onagraceæ, Salpiglossis atropurpurea in Solanaceæ, and Lechenaultia in the Goodeniaceæ, mentioned by Hassall, are also fourfold. We also find some in groups of four, but not so completely united; as in Beschorneria, and a specimen of Fourcroya, mentioned by Schacht. The other Agaves I have examined are simple. Typha (No. 26), Cytinus (No. 57), are also 4-celled. The Acacieæ are many-celled, nine to sixteen cells united in cubical or sub-trigonous forms. The Acanthaceæ are perhaps the most beautiful of all, in many forms : some in a peculiar coil, which can be unwound; some in an oblong coil ; some not coiled, but richly marked; some spherical (No. 108-127, P. vii. viii. xix.); examined in the $\frac{1}{4}$-inch objective, occasionally in the 8th objective.

I here offer my thanks to Dr. Hooker for allowing me to collect pollen in the Kew Gardens, and to Mr. Oliver for permission to collect it on some dried specimens, and to Dr. Murie for his valuable assistance. 
The accompanying list of plants shows those of which the pollen has been described. Those marked with an asterisk I have myself examined and measured. Opposite the name of the plant is the size $\frac{5 \text { to } 60}{6000}$ ths of an inch, measured by the micrometer.

The authors who have examined pollen are:

Purjinke (P.), in Latin.

Mohl (M.), the first No. in the German edition, the second from the French translation in the 'Annales des Sciences Naturelles.'

Fritzsche (F.), in German.

Schacht (Sch.), 'Jahrbuch Bot.,' Berlin, ii., in German.

Nagili (N.), ib. iii.

Romanoff (R.), ib. vii.

Pollenden (Pol.), Bonn.

Leursen (L.), in 'Jahrbuch Bot.', Berlin, vii.

A. Bennett (B.), in the 'Science Review.'

Worthington Smith, in the 'Gardeners' Chronicle' (re-published in 'Microscopical Journal'). WS.

Hassall, in 'Annals of Nat. Hist.' ix. 1834. H.

'Micrographical Dict.' (M. D.)

Lindley, in 'Introduction to Botany' (Lind.).

Dr. Hooker, in 'Sikhim and New Zealand' (H. f.).

Decaisne, in the 'Bruxelles Académie,' t. xiii. 1837.

The drawings in these plates are all done to scale $\frac{1}{12}$ th of an inch $=\frac{1}{6000}$ th or $\frac{1}{500}$ th of life size.

EXOGENS.

Granrnes. According to Mohl, they are 'oval, shining, not viscous, finely grained, with a punctiform pore and a narrow halo.' Hassall says, 'granules separate, circular at first, but on the emission of a single pollen tube, with which each grain is furnished, generally hecoming ovoid.' Fritzsche describes the pollen as having a valve to the pore. I find them somewhat prismatic, tapering at both ends, rounded, one smaller than the other, without viscidity. I have been unable to detect the pore. I have examined many species, but find only three which are not prismatic, which are the only species of the Paniceæ which I have examined. The pollen flies off so rapidly on the opening of the anthers, that we must secure the pollen as the anthers open.

Oryzea.

* Oryza sativa, 6.

Phalaridea.

Zizania aquatica. $\mathrm{H}$.

*Zea Mays, 20. 23. M.

*Coix Lachryma, 15.

*Sorghum saccharatum, 11. M.

*Holcus mollis, 7 .

*Phalaris canariensis, 7. M.

- picta. P. 1. 12. 
Phalaridea-contd.

Phleum nodosum: Mir. xxxi. 36.

*Alopecurus pratensis, 10 . F.

* _ arvensis, 7.

- geniculatus. F.

- nigricans. F.

Panicea

Colobachne vaginata. $\mathrm{F}$.

Panicum palmifolium. $\mathrm{H}$.

*_ plicatum, 7. 8.

*Streptium floribundum, 6.

Milium vernale. F.

Agrostidea.

Agrostis stolonifera. P. i. 11.

Polypogon, sp. H.

Arundinea.

Arundo littorea. $\mathrm{H}$.

Phragmites communis. M. H.

Chloridea.

Spartina juncea. $\mathrm{H}$.

Avenea.

Avena fatua. $\mathrm{H}$.

* - sativa, 8.

latifolia. P. 1. 17.

- subspicata. F.

Aira vaginata. $H$.

*___ cæspitosa, 5 .

*Anthoxanthus odoratus, 10 . F.

*Lagurus ovatus, 7.8.

Festucea.

Bromus erectus. P. 1. 46.

Melica altissima. P. 1. 15.

Sesleria elongata. $\mathrm{H}$.

Poa fertilis. $H$.

*__ pratensis, 5 .

* __ annua. B. cxix. 8. F.

- alpestris. F.

Festuca pallens. P. 14.

* ovina, 6.7.

- elatior. M.

*Dactylis glomerata, 6. M. vii. ix. 29. B. cxix. 10. F. abbreviata. $\mathrm{H}$. cæspitosa. H. Ant. cxxxvii.

*Arundinaria falcata, 9. WS. 53.

Hordea.

*Triticum æstivale, 12.

hybernum. $\mathrm{H}$.

rigidum. $\mathrm{H}$

Spelta. M.

*Lollium perenne. $\mathrm{H}$.

Egilops squarrosa. M.

Elymus strictus. $\mathrm{H}$.

sabulosus. P. 10. H. Sch. iv. 14.

Andropogon furcatus. $H$.

- strictus. M.

*Pogonatherum, 9.

Saccharum officinale. Sch. xviii. 20. 
CrPeracex. According to Mohl, drop-shaped; according to Hassall, 'ovate lanceolate.' I usually find it somewhat cubical or 5-angled or subglobular.

\section{Caricece.}

*Carex præcox, 8. 10. .F.

* - pendula, 10.

* a arenaria, 8. 9.

- tomentosa. $\mathrm{H}$.

- Ederii. H.

- acuta. $\mathrm{H}$.

- cyperoides. M.

- muricata. M

- paludosa. $\mathrm{F}$.

- cæspitosa. F.

Schonidere.

Fraseri. F.

\section{Cladiea.}

Schoenus mucronatus. P. i. 17.

Cladium germanicum. M. vi. 33.

Scirpece.

Dulichium spathaceum. M.

Scirpus atrocinus. $\mathrm{H}$.

- triqueter. $\mathrm{H}$.

- capitatus. $\mathrm{H}$.

- palustris. M. vi. 32.

Isolepis Holoschœnus. M. vi. 9.

- romanus. P. 1. 18. Lin. iv. 16.

*__ gracilis. 4.5.

Cyperus.

Eriophorum polystachyum. P. 1. 19.

Cyperus longus. $\mathrm{H}$.

* ustulatus, 4.

* _ alternatus, 5. 6.

Papyrus antiquorum. M. vi. 2.

Resticeæ (Mohl). Oval punctate.

Restio fruticosus. M.

Eriocaulonex. Unexamined.

TrPHACEE. According to Mohl, 'globular, with a pore;' according to Hassall, and as I have observed, 'four oval grains in one plane.'

*Typha latifolia, 8.9. H. xii. 5.

angustifolia. P. 1.4. M.

- minima. M.

Sparganium ramosum. M.

simplex. M.

ArACEx. According to Mohl, globular pleats or pores; Hassall gives elliptic, or a flat with a parallelogram. I find spherical, or oval with the numerous tubes emitted from the ends.

*Amorphallus, 10.

*Richardia æthiopica, 12. P. 1. 3. H. xiii. 7. Lin. iv. 13. B. cxix. 53.

albomaculata. WS. 37.

Calla palustris. H. xiii. 6 .

Caladium biçolor. F.

- seguinum. M.

Anthurium Hookerii, 3. 4.

* _ cordatum, 3.

- Scherzianum. WS. 36. 
Aracea-contd.

Anthurium Patrinii. WS. 34.

Spathophyllum heliconiæ folium. WS. 38.

Phyllotænium mirabile. WS. 33.

Arum divaricatum. M. xvi. 8. M.

- furcatum. P. 1. 2.

- ternatum. M.

Pothos crassa. P. xvii. 5. longifolia. M.

*Rohdea Japonica, 6.

*Pennillia turpiflora, 6.

Pandanez. Mohl, oval with one furrow.

JUNCACEA. According to Mohl, in tetrahedons, in fours; according to

Hassall, $(a)$ in fours,three placed in one plane; $(b)$ rough, subglobular, with three bands; $(c)$ oval, echinulate, with one pore.

Juncus articulatus. H. xiii. 8.

*__ effusus, 6 .

Jacquini. M.

Helonias latifolia. P. ii. 9. crenata. H. f. Ant. xlvii.

*Luzula campestris. F. ii. 11. B. cxix. 4. vernalis. M.

*Xerotes longifolia, 5 .

*Astilbe Cunninghamii, 6.

pumila. H. f. Ant. cxxvii. echinulata. H. f. Ant. cxxviii.

XYRIDEe.

Xyris erianthus. M.

Bormaniacex. alpestris. M.

Tripteralla violacea. M.

Commelynes. According to Mohl, elliptic, with one furrow, as I have observed; Hassall says it is somewhat curved.

Tradescantia virginica. F. P. 1.2. M. i. 20. H. xi. 10.

* _ variegata, 12.

* _ discolor, 5.

* - Warzeloides, 6 .

Commelyna cælestris. P. 1. 21. tuberosa. Mir. xxxi. 45 .

*Oochleonema, 10.

*Dichorazandra, 6.

*Palisotia Barlerii, 5. 6.

Pontederacex. Simple ellipse; according to Mohl, with two longitudinal furrows.

*Pontederia cordati, 9. 10. M. i. 25.

LILIACEx. Usually a simple ellipse with one slit, smooth or prickly, ornate or reticular. II. Trigonous.

Aloe obscurus. H.

- mararitiferus. M.

- saponarius. $\mathrm{H}$.

*__ mitriformis, 12. M. i. 22.

*__ pluridens, 12.

*__ linealis, 10.

*__ abyssinicus. WS. 40.

- frutescens. F.

*Howarthia radula. $H$. reticulata. $\mathrm{F}$.

-Asparagus officinalis, 8. H. xii. 11. 
Liliacea-contd.

*Asparagus scandens, 8.

*Arthropodum fimbriatum, 10. H. F.

Anthericum aloides. M.

- ramosum. $\mathrm{M}$.

Albuca minor. M. ix. 6. P. 1. 16.

Allium fragrans. $H$.

- fistulosum. M. i. 9.

*Agapanthus umbellatus. $\mathrm{H}$.

*Asphodelus Bulbine, 20.

* _ cervis, 10.

* _ fistulosus, 3. Mir. xxxi. 34.

*Brodiæa, sp. 20.

*Bulbine frutescens, 10.

- longiscapa. M.

- prostrata. $\mathrm{H}$.

*Bessera elegans, 10.

Bulbocodium vernum. F. 23.

*Chlorogalum, sp. 13.

*Chlorophytum, sp. 10.

* _ comosum, 8.

*Conenthanthera Echardii. M. i. 19.

*Convallaria bifolia, 6 .

*- siberica, 11.

* - majalis, 7.

*_ Polygonatum, 10. P. iii. 11. H. WS. 42. F. P. iii. 12.

B. cxix. 19.

multiflora. F.

*Cordyline Rodii, 8.

Chrysabactron Rossii. H. f. Ant. xlv.

*Cyananthera, sp. 10.

*Oyrtonema lutescens, 16.

*Colchicum autumnale, 12. M. vii. 8. 50.

Czackia Liliastrum. $\mathrm{H}$.

*Drymiopsis, sp. 10.

*Dracæna cernua, 14.

* - Nova Caledonica, 9.

*__ magnifica, 6.

*Dianella strumosa, 5. Trigonous cocked hats.

*__ cærulea, 6. F. 31.

nemorosa. F.

*Erythronium americanum, 20.

*Eucomis stricta. H.

— regia. M. undulata. M.

Fritillaria imperialis. F. M. P. iii. 13. B. cxix. 34. WS. 43.

*_ meleagris, 18 . F. M. B. cxix. 35 .

- glaucescens. F.

- pyrenaica. $\mathrm{F}$.

- latifolia. $\mathrm{F}$.

- lutea. F. persica. F.

Funkia Seiboldii.

*Gloriosa superba, 6.

Hemerocallis flava. $H$.

fulva. P. iv. 2. M. i. 7. ix. 8.

graminea. P. iv. 3.

japonica. $\mathrm{N}_{\text {n }}$ 
Lilincea-contd.

*Hyacinthus amethystinus, 13.

* orientalis. F.

*Knyphopia uvaria.

*Lachelania luteola, 6.

*Lilium philadelphum, 20.

*__ auratum, 15.

*__ japonicum, 15.

*__ Kramerii, 15.

*__ Humboldtii, 15.

* _ monadelphum, 18.

*__ canadense, 20.

*__ sericium, 20.

- longifolium. H. xiii. $13 . \quad$ WS. 38.

— californicum. WS. 39.

- album. $\mathrm{H}$.

— tigrinum. H. M.

- candidum. $\mathbf{M}$.

- Martagon. M. Pol. iii.

— Davidi. Elwes. Lil.

- bulbiferum. M.

*Massondia lancifolia, 8.

*Myrsiphyllum falciforme, 9.

*Narthecium ossifragum. WS. 1.

*Ornithigalum nutans, 10.

— villosum. $\mathrm{F}$.

— umbellatum. F. refractum. F.

* Ophiopogon japonica, 7. 8.

${ }^{*}$ Puschinia scilloides, 7 . F.

*Plesiocanthus Teta, 9. H.

*Ruckia elesmata, 10.

*Streptopus argentifolius. F.

*Stipandra pulchella, 7.

* - cæspitosa, 7 .

*Scilla peruviana, 12.

*__ nutans, 12.

* _ campanulata, 8.

- siberica. $\mathrm{F}$.

- bifolia. F.

*Tritelia unifolia, 7 . laxa.

*Trichonema speciosa, 17.

*Tritoma, sp. 12.

*Tulipa gesneriana, 12. P. iii. 13. H. F.

* - suaveolens, 12. F.

* - sylvestris, 10.15. F.

*Uvularia, sp. 10.

*Veltheimia viridiflora, 12 . M. F.

*__ uvaria, 18. P. iv. 6.

* _ sarmentosa. F.

*Yucca filamentosa, 10. 12. H.

HYPOXIDE gloriosa. Sc. xviii. 6.

*Hypoxis obliqua, 10. prolifera. P. ii. 7 . stellata. $\mathrm{H}$.

Curculigo recurvata. F. M. 
Dioscoreacee. According to Mohl, oval, one furrow; or B. with two furrows.

*Tamus communis, $10 . \mathrm{H}$.

Dioscorea villosa. M.

- aculeata, $\mathbf{M}$.

SmILACEx. According to Mohl, elliptic, with long furrow, simple or punctate; in Trillium, a smooth sphere; in Philesia, with echinate sphere.

*Smilax, sp. 14.

*Trillium erectum, 8. F. grandiflorum. F. pictum. F.

Paris quadrifoliata. P. ii. 11. F.

polyphylla. H. f. Sikkim. 24.

*Philesia buxifolia, 15.

*Lapageria alba, 17. F.

Patme.e.

Ruscus Hypoglossum.

${ }^{*}$ Chamærops Hystrix. F.

- Palmetto, 5-7. P. iv. 7. M. humilis. F.

*Areca Baurii, 12.

Kunthia speciosa. H.

Chamædoria Schiedeari. F.

* _- Harwigii, 5.

*JUNCA GENEA. Elliptic or globular, rough or echinate.

*Triglochin palustris, 5 .

*Potamogeton crispum, 10.

* n natans, 7 .

*Aponogeton distachyum, 8.

Buтомеж. Globular echinate or simple ellipse.

${ }^{*}$ Hydrocleis Humboldtii, 6-8. H. xiv. 9.

Stratiotes Aloides. P. xxvii. 5. M.

Butomus umbellatus. H. M.

Alismee. Polyhedric.

*Alisma Plantago, 8. P. i. 5. M. iv. 12. M. D. xxxii. 10.

BromeltaceE.

Billbergia thyrsoides. M.

- amœna. H.

- patentissima. M.

*__ pallida, 12.

* _ nutans, 15.

Cottendorfia florida. M.

*Pitcairnia integrifolia, 20. latifolia. M.

*Tillandsia, 15.

AMARYLLIDE. E. Elliptic, smooth, or reticulated.

Amaryllis gigantea. M. i. 24.

- blanda. M.

- miniata. M.

— formosissima. M. F.

- Reginæ. F.

- rutilans. F.

- purpurea. $\mathrm{H}$.

- undulata. M.

*Clivia nobilis, 10.

Crinum amabile. H. xii. 20. 
Amaryllidea-contd.

*Crinum ornatum, 20.

*— sp. 16. pratense. WS. 59.

Craveyanum. F.

${ }^{*}$ Chlidanthus fragrans, 27.

*Eucharis, sp. 19.

— alliecum, 12. 20.

*Imantophyllum miniatum, 15. Aitonii. $\mathrm{H}$.

*Galanthus nivalis, 6. H. WS. 6. M. F. Clusii. F.

Griffinia hyacinthoides. H. M.

*Hæmanthus coccineus, 12.

*_ puniceus, 15.

* — pubescens, 13. tigrinus. M. H.

*Hippoeastrum alatum, 15.

*Leucojum vernalis, 6. M. F.

*Narcissus poeticus, 8, 12. B. cxix.

* J Jonquilla, 20.

* _ triandrus, 10.

- radiiflorus. $\mathrm{F}$ longifolius. F.

*Nerine undulata, 18.

*- sarniensis, 18.

*Pancratium Tagetta, 30.

* - caribæum, 45.

* _ declinatum, 38. H. xiii. 24. Lin. iv. 17. maritimum. M.

"Zephyrinus grandiflnrus. H.

*Alstræmeria Curtisiana. M. vi. 21. Ordo 20. psittacina. H. M.

*Agave americana. M.

* - curvifolia, 14. 15.

* - lurida. M.

*Beschorneria Tomelli, 25.

*Fourcroya, 12.

- longæva. M.

*Vellozia aloefolia. M. v. 19.

HAMADORE with various forms in the same anther.

Anisogeisus rufus, 18. flavus. H. xiii. 19. M. coccineus. M.

(Barbacinia) glauca. M. ignea. $\mathrm{M}$.

Wachendorfia paniculata. M.

Hemadorum planifolium. M.

TACCACEA. A broad ellipse, one opening.

*Tacca artocarpifolia, 14.

*Atacca cristata, 25.

IRIDEE. Mohl gives many forms-elliptic, globular, smooth, echinate, or spiral.

*Antholyza æthiopica, 24. H. M.

* Crocus lacteus, 16. 22.

* - biflorus, 15. 20.

* _ sulphureus. No pollen-barren. 
Iridea-contd.

*Crocus stellaris, 8. Imperfect pollen.

*- sativus, 25-28. Echinulate. H. xiv. 26.

* - insularis, 16. 20. F.

- aureus. 20.63. Echinulate. WS.

* - revolutus, 15. Echinulate.

*_ vernus, 15. 20. Echinulate. H.

- albiflorus. Echinulate. M.

*__ nivalis (Sieberi, Gay). Spiral.

* - mæsiacus. Spiral. M.

* - susianus. Spiral.

*__ argenteus. Spiral.

Adenoropium villosum. M.

*Cyclobothra, sp. 10.

convallarioides. $\mathrm{H}$.

*Cypella gracilis, 12.

*Diasia graminifolia, 12.

Gladiolus segetum. Sc. xviii. 2.

*__ floribundum, 25.

- communis. M.

- tristis. M.

- insignis. $\mathbf{M}$.

Iris florentina. M. $\mathrm{H}$.

— ruthenica. M.

- flavescens. M.

*__ Pseud-Acorus, 28-30. Pol. iii.

*__ sinensis, 12.

* _ fimbriata, 18.

*__ elatior, 25.

- germanica. M.

- iberica. WS. 61.

- Kœnferii. WS. 60.

- Güldenstaedtii. M.

Ixia florida. M.

- deusta. M.

- pulcherrima. M.

- polystachys. F.

Micranthus alopecurioides. M. plantigenius. M.

fistulosus. M.

Moræa racemosa. $\mathrm{H}$.

Ovieda corymbosa. M.

*Schizostylus coccineus, 15.

*Sisyrhinchium magellicanum, 8.

convolutum. M. luteum. F.

*Tritonia, sp. 15-18.

Tigridia Pavonia. M. i. 23.

*Synnotia bicolor, 21.

*Watsonia irioides, 4. H. xiii. 6.

- plantaginea. H. M.

lucidior. H. M.

Musacex. Spherical.

*Musa paradisiaca, 20. Troglodytarum. M.

*Strelitzia Reginæ, 25. H. xiv. 30. Sc. xviii. 9. F. M. humilis. H.

Marantacex. Spheroid, smooth, or echinate. 
Marantacea-contd.

Canna occidentalis. F. H.

* indica, 12. M. F. ix. 6. H. xiv. 23. B. cxix. 51. limbata. F.

- Sellowii. F. pedunculata. $F$.

patens. F.

- chinensis. F.

*Phrynium sanguilentum, 30. S. xviii. 1.

* Calathea bicolor, 15.

*Ischonosyphon Aroura, 7.

ZINGIBERACE . Globular echivate.

Alpinea, sp. $\mathrm{H}$.

Costus, sp. 15.

Hedychium suaveolens, 15.

- Gardnerii. $\mathrm{H}$.

- flavescens. M. H.

- coronarium. $\mathrm{H}$.

EXOGENES.

Roscoea purpurea. $\mathrm{H}$.

CrCadra.

Zamia longifolia. S. xvii. 26. P. xviii. 4. media. P. i. 1.

*Stangeria, sp. 12.

*Ceratozamia, sp. 10.

Welwitzia mirabilis. Hf. Tr. Lin. vi. 13.

CoNIFrexe. I. Two lobes connected by a broad band.

II. Subspheric, with 3 or 4 coats.

III. Simple oval.

IV. Oval, with 6 furrows.

I. Pinus sylvestris. F. H. xiv. 34. S. xvii. 7. Pol. ii.

- novazealandica. $\mathrm{H}$.

- Tæda. $\mathrm{H}$.

- rufa. P. v. 14.

- Pinaster. F. M.

* L Laricio, 15.

* __ excelsior, 14.

- Cembra. M.

uncinata. M.

Abies excelsa. M. ii. 32.

- pectinata. S. xvii. 9.

Picea vulgaris. S. xvii. 16.

Dacrydium Franklini. Hf. Tas. O. B.

Microcachrys tetragona. Hf. F. C. A. 3 lobes.

Pherosphera Hookeriana. Hf. Tas. I. C. 3 lobes.

Cedrus Libani. WS. 50.

II. "'Larix communis, 8. S. xvii. 6. M. F. Pol. i.

- Griffithii. Hf. Lin. 21.

*Oryptomia, 8.

*Sequoia sempervivens, 15.

* - gigantea, 5 .

*Juniperus communis, 10. P. v. 11. M. H., Pol. iii.

- oxycedrus. F.

- virgineana. $\mathrm{F}$.

Sabini. H.

*Thuia orientalis, 10. S. xvii. 22.

occidentalis. F. M.

Diselma Archeri. Hf. xcriii. Tas. 
Conifer $a$-contd.

"Cupressus sempervivens, 6. S. xvii. 17. F. M.

* Benthamii, 7.

Frenela australis. P. v. 13.

— nuktensis, 7. Hf. Tas. xevii.

Podocarpus Salviniana. S. xvii. 12.

Araucaria brasiliensis. S. xvii. 25.

imbricata. M. i. 2.

Cunninghamia sinensis. M.

*Taxus baccata, 7. F. M. i. 5, x. 61. H.

Callitris quadrivalvis. F.

III. Gingo (Salisburia) biloba. M. i. 11. S. xvii. 15.

IV. Ephedra major. S. xvii. 13.

distachys. M., with 6 slits.

fragilis. M.

Betulaces. Subglobular or polyhedric, 3, 4, or 5 pores.

${ }^{*}$ Betula alba, 6. B. cxv. 2. F. H.

*Alnus glutinosa, 5 . M. H.

SAurCACEx. I. Elliptic, with 3 slits and bands. II. Globular.

*Salix viminea, 6 . F. M. H.

* nigra, 4.

* _ nigricans, 6.

- riparia. M.

*__ retusa, 11. B. cxiv. 50.

*__ phylicifolia, 7.

* _ hastata, 6.

* _ capræa, 5. 6.

- triandra. M.

*__ pentandra, 10.

- cinerea. P. v. 17.

- alba. F.

polymorpha. F.

*Populus nigra, 6. 7. balsaminea. B. cxix. 3.

- alba. P. v. 18. Lind. iv. 18.

CoRruaces. Elliptic with 3 bands, or spherical with 3 pores, or polyhedric with a pore on each facet.

*Fagus sylvatica, 6-7. M. H.

*Castanea vescaria, 5. 7. H.

*Ostrya vulgaris, 6 . M. $\mathrm{H}$.

*Carpinus Betulus, 5. M.

*Corylus Avellana, 6. F, i. 6. M. H. B. cxix. i. N. Pol. 37.

Quercus Robur. M. H.

pedunculata. $\mathrm{F}$.

*__ sessiliflora, 10.

Mrricaceze. Cerris, 9. 10.

Myrica Gale. M.

- cerifera. M.

— æthiopica. M.

- quercifolia. M.

Comptonia asplenoides. M.

Platanacex. Minute, elliptic with 2 slits.

*Platanus orientalis, 3. B. cxix. 18.

\section{Castuarinez.} acerifolia. F.

Casuarina equisetifolia. P. iv. 9. M. indica. M. 
Casuarinea-contd.

Casuarina quadrivalvis. M.

stricta. M.
ULMAcex. Globular, rough. Mohl gives 3 forms.

*Ulmus campestris, 7. 8. M. H.

* - montana, 8.

Celtis australis. M:

Moracex. Globular, with 12 pores. Mohl gives other forms which I have not seen.

*Dorstenia maculata, 4.

Coussapoa latifolia. M.

Broussonetia papyrifera. M.

Pourrina velutina. M.

bicolor. M.

- cecropiæfolia. M.

Cecropia peltata. M.

Lacistema serrata. M.

Cannabinze. Ovoid with triangular end.

Humulus Lupulus. M.

*Cannabis sativa, 7. 8. P. v. 20. M. iii. 21.

URTICACEA. Spherical, or angular, sub-cubical.

*Urtica dioica, 4. P. v. 19. Lin. iv. 20.

* — urens, 3. M. WS. 54.

*Parieteria officinalis, 3 . M. erecta. M.

*Bœhmeria nivia, 3.

- caudata. M.

*Pilea microphylla, 3.

*-_ muscosa, 5 .

Juglatdace . Globular. Hassall has observed 'twin tubes issuing in a round centre of the granule; 'but I have not been able to see them.

*Juglans regia, 10 . M. F.

- oliviformis. M.

Asaracex. Mohl gives as globular without pore or slit.

Asarum europæum. P. v. 21. M.

*Aristolochia Clematidis, 9. P. xvii. 28.

*__ Brasiliensis, 9. 10.

trilobata. M.

Podostemacex. Oval with 3 slits.

Lacis fucoides. M.

Crtinacez. Mohl, I. oval with 3 slits; II. elliptic with 3 pores. I find Oytinus with 4 fold-slits.

*Oytinus Hypocistis.

Cynomorium. M.

Langodorffia hypogæa. M.

EUPHORBIACE必. Mohl, spherical granular or cellular or punctate, or elliptic with 3 bands and furrows.

*Croton humile, 15.

pictum. WS. 20.

- pseudochinum. M.

- punctatum. M.

- Tiglium. M.

*Jatropha podograda, 18.

- Manihot. M.

— pandurifolia. M.

- urens. M.

*Dalechampia, sp. 10.

*__ Rœzliana, 15. 
Euphorbiacea-contd.

Aleurites triloba. M.

*Sarcoccus trinervis, 8.

*Buxus sempervirens. M. B. 6.

* Wallichiana, 7.8.

Poranthera linarioides. M. arbuscula. M.

*Ricinus communis, 7. M. lividus. P. xvii. 13.

${ }^{*}$ Euphorbia (Poinsettia) pulcherrima, 10.

* exigua, 6.

*__ Paralias, 9. 13.

* collatioides, 10.

*-_ oxystegioa, 11.

*_ Bojeri, 10.

*__ bupleurifolia, 16.

*__ Heliscopia, 8. 10.

Peplus. M.

*_ fulgens, 10 . $\mathrm{M}$

- verrucosa. M.

- Cyparissias. M.

palustris. F.

Characias. F.

Caput-Medusæ. F.

*Riedia glaucescens, 3 .

Cluytia pulchella: F. alaternoides. M.

*Mercurialis perennis, 3. B. cxix. 5 . annua. M. WS. 20. elliptica. F.

*Acalypha Commersonii, 2, 3.

- acuta. M.

- acuminata. M.

- -- scabrosa. M.

*Sebastiana corniculata, 6. 9.

Xylophylla glauca. WS. 21.

Sapium casuarieæfolium. M.

Cicca distachya. M.

Securinega nitida. M.

Cnemidostachys tragiodus. M.

- longifolia. M.

EupEtreacex. According to Mohl, inserted in fours, but I have found them disunited, globular, with 3 pores and bands.

Empetrum nigrum, 4. 5. M.

Proteacex. Those connected with a pore at each corner.

Aulax pinifolium. P. v. 15.

Anadenia Manglisii. $\mathrm{H}$.

*Banksia integrifolia, 9.

- marginata. $\mathrm{H}$.

- verticellata. $\mathbf{H}$.

speciosa. H.

Dryandra formosa. H. F. longifolis. $H$. F. armata. $H$.

${ }^{*}$ Grevillia lancifolia, 15.

* ros marinifolia, 10. F. i. 1.

*__ Thielmannii, 12. 
Proteacea-contd.

*Grevillia microstylis, 15.

* - montana, 10.

- linearis. M. H.

- sulphurea. $\mathrm{H}$.

- pubescens. F.

setacea. F.

mucronata. F.

Baueri. F.

*Hakea nodosa, 10.

*_ Stroudii, 15.

- pedunculata. H.

- pugioniformis. $\mathrm{H}$.

- acicularis. F.

- elliptica. F.

dactyloides. F.

Leucadendron hirtum. F.

Isopogon anemonifolius. $\mathbf{H}$.

*Persoonia juniperina, 12.

Protea multiflora. M.

- acaulis. M.

Rhopala serrata. M.

- heterophylla. M. rhombifolia. M.

LAURINEA. Globular, rough; no pores.

*Laurus nobilis, 7.

— occidentalis. P. v. 24.

carolinensis. F.

*Tetranthera japonica, 25.

Santalacez. 3 slits.

Oxyris alba.

Eleagneacex. 3 or 4 cornered, or ovoid with 3 pores.

*Eleagnus europæus, 16 . M.

- angustifolia. M.

Hippophae rhamnoides. M.

Thymeize. Globular, rough, with no pores.

*Daphne Laureola, 6.

* odora, 7.

* Cneorum, 6.

* Mezereum. 7. F. M. H.

- pontica. F.

- collina. F.

* _ indica, 8. alpina.

*Pimelea bandensis, 8.

- hispida. $\mathrm{H}$.

- decussata. H.

- ligustrina. M.

drupacea. F.

Passerina pectinata. F. hirsuta. F.

canescens. M.

*Gnidia daphnoides, 8. M.

— simplex. M.

- virescens. F.

Lachnea penicellata, 7. 8. purpurea. F.

Dais cotynifolia. M. 
Thymelece-contd.

Dais madagascariensis. M.

Pen facez. 8 or 6 slits.

Penæa mucronata. F. ii. 24. M.

- squamosa. M.

Polygonacex. (a) globular echinate; (b) oval, with 3 bands and slits; (c) somewhat trigonous.

Polygonum emarginatum. P. xiii. 7. Globular.

- chinense. M. Globular.

*__ amphibium, 10. Globular.

*_- Hydropiper, 10. Globular.

* Persicaria, 6. Globular.

* _ Bistorta, 8. M. v. S. Elliptic.

— cymosum. M. v. 4.

- ambiguum, 8.

*__ Tamus, 5.

- dumetorum. M.

- undulatum. F.

* - Aviculare, 4.

Coccoloba curtipendula. M.

*Eriogonum flavum, 9.

*Rheum undulatum, 8. F.

* - officinale, 8.

- hybridum. M.

- nobile. Hf. Sik. 15.

ponticum. P. xiii. 6.

* Rumex acetosella, 7.

—_ acetosum. M. P. xii. 5.

_ obtusifolium, 7.

- scrutatum. M.

Atraphaxis undulata. M.

- spinosa. M.

*Muhlenbachia complexa, 9. 10.

Amaravinace. . Polyhedric, with many pres.

*Hablitzia tamifolia, 3. 4.

* Celosia cristata, 12.

*Aevra sanguilenta, 2.

*Amaranthus tristis, 6.

- caudatus. M.

- paniculatus. M.

Iresine diffusa. P. xiii. 13. Lind. 19.

Alternanthera Achyranthes. M.

Chamissoa acuminata. M.

Bucholetzia maritima. M. iv. 4.

SAlsolacex.

Blitum capitatum. M. iv. 13.

Chenopodium Botrys. M.

*_ album, 7.

- glaucum. M.

* — Bonus Henricus, 6. P. xiii. 11. M. ambroisoides. M.

Beta trygyna. P. xiii. 12. M. maritima. M.

Salsola scoparia. M. iv. 12.

- Kali. M.

Salicornia fruticosa. M.

Suæda salsa. M. 
Salsolacea-contd.

Bassella alba. M. ii. 12. Md. xxxii. 19. Cubical.

Paronychiaceza.

*Corogiola littoralis, 2.

*Scleranthus aureus, 4.

Piperaces. Small elliptic. Mohl.

Piper emarginatum. P. v. 20.

Puritum. M.

- syringæfolium. M.

LORANTHACEx. (a) globular with 3 slits; (b) elliptic; variously disposed in Mohl.

Viscum album. M. Decaisne, Brux. Acad. v. xiii. ii. 17.

- anceps. M.

Loranthus bicolor. M. iv. 3.

— robustus. M. iv. 2.

- polyanthus. M.

- nitens. M.

- falciformis. M.

- elegans. M.

- lineatus. M.

- patens. M.

- crassifolius. M.

Misodendrum punctulatum. Hf. Ant. civ.

- brachystachium. Hf. Ant. cv.

Nrctagrnez. Spherical, rough, with many pores.

*Pisonia Olferisii, 7 .

- spectabilis, 8.

*Bougainvillia,

*Mirabilis Jalapa, 24. M. P. v. 21. Lind. iv. 19. 'H. xvi. 92. longifolia. M.

Calymeria (Oxybaphus) viscosa. M. iv. 17.

Allionia nyctaginea. M.

Phytolaccaceж. Globular, smooth, or polyhedric.

*Petiveria alliacea, 5.

Rivina humilis, 6.

* - lævis, 7 .

* - flava, 8 .

- brasiliensis. M. ii. 34 .

Phytolacca deandra. M.

- abyssinica. M.

GAMOPETALEÆ.

Plantaginex. Accordirg to Mohl and Hassall, spherical with many pores; to me they appear polyhedric.

*Plantago lanceolata, 6. H. xv. 64. M.

- oblongifolia. $H$.

*__ Coronopus, 5.

*— major, 6.

* media, 6.

*__ Cynops, 8. Wulfenii. M.

*Littorella lacustris, 4.

Labiatæe. Elliptic or cylindric, with 3,4 or 6 bands.

*Physostegia virginica, 10. 12. H.

*Westringia eremicola. $\mathrm{H}$.

*Scutellaria coccinea, 6 .

*__ alpina, 6.

*_ galericulata, 7 . H. 
Labiata-contd.

*Scutellaria lupulina. $\mathbf{H}$.

* __ violacea, 7 .

scordifolia. M.

- hastifolia. M.

Moccosini. WS. 79.

*Galobdolon luteum. H. F.

*Galeopsis versicolor, 15.

*__ Tetrahit, 9 . M. ii. 3.

*Lamium purpureum, 4. B. cxix. 16. F.

* album, 9. B. cxix. 15. F.

* maculatum, 8. F.

— amplexicaule. F.

* Stachys palustris, 11 .

iberica. $\mathbf{H}$.

- setifera. $\mathrm{H}$.

coccinea. $\mathrm{H}$.

— rugosa. P. vii. 3.

* _ Betonica, 13.

*Phlomis fruticosa, 15. 18.

Nissolii. M.

microphylla. M.

*Leonurus Cardiaca. 8.

nepalensis. $\mathbf{M}$.

Ballota nigra. $\mathbf{H}$.

Lycopus europæus. H. M.

Narrubium vulgare. $\mathrm{H}$. hispanicum. M.

Molucella lævis. $\mathrm{H}$.

*Lophanthus rugosus, 8.

Teucrium lucidum. $\mathbf{H}$.

- pyrenaicum. $\mathrm{H}$.

- hyrcanicum. M.

— Chamædrys. M. ii. 16.

- Scordium. M.

* Scorodonia, 12.

montanum.

*Ajuga reptans, 9.

* - pyramidalis, 9. M.

Coleus, sp. 8. 11.

*Sideritis foetida. $\mathrm{H}$.

- taurica. $\mathrm{H}$.

* - megastachya, 8. M. ii. 13. ix. 15. 4-celled.

- scordioides. $\mathrm{H}$.

Salvia interrupta. P. viii. 3. Lind. iv. 24.

— glutinosa. M. ii. 13. ix. 15.

* __ nubicola, 10.

* — patens, $10 . \quad$ WS. 78.

*__ coccinea, 10.

* - verbiacea, 6.

sclarea. H.

- splendens. $\mathbf{H}$.

*Lavandula spica, 6 . M.

Thymus Serpyllum. M.

Acinos. M.

grandiflorus. M.

* Mentha rotundifolia, 10.

*—_ piperita, 6 . 
Labiate-contd.

Mentha crispa. M.

*Prunella vulgaris, 10. M.

Gardoquia multiflora. H.

*Origanum vulgare, 12.

*Satureja rupestris, 6. M. ii. 18.

*Monarda fistulosa, 10. 11. H. Mir. xxxi. 30.

*Calamintha officinalis, 8. 12. M.

*Plectranthus australis, 8. 10.

*Dracocephalum grandiflorum, 14.

— speciosum. H.

peregrinum. P. vii. 6.

*Nepeta Mussini, 9.

- violacea. M. Ws. 77.

*__ Glechoma, 6. B. cxix. 11. F.

Hyptis Séllowi. F.

${ }^{*}$ Hyssopus officinalis. M.

*Pognstemum plectranthroides, 8. F.

*Prostranthera incisa, 12. - violacea. F. i. 11.

*Ocymum sanctum, 8.

basilicum. H.

Ziziphora serpyllacea. P. vii. 7 .

Aolanthus suavis. M.

*Melittis Melissophyllum, 9 .

Verbenaces. According to Mohl; in four kinds: (a) triangular,

$(b)$ ovoid, $(c)$ spherical punctured, or $(d)$ divided in cubes. I also find $(e)$ flat and $(f)$ circular forms.

*Verbena Aubletii, 10. MI. iii. 20.

* L littoralis, 12. 13.

* - officinalis, 10.

* - hispida, 14. 15.

*__ aculeata. M.

Lantana aculeata. H. iii. 17.

* _ nodosa.

Lippia dulcis.

*Stachytarpheta bicolor, 48. 50.

*Verbena Melindri, 10.

Tectona grandis. M.

Vitex agnus castus. M.

lanaginosus. M.

Negundo. M.

*Aloysia citriodora, 5 .

*Lantana flava, 8. crocea. $F$.

*Clerodendron Thompsonianum, 18. 20.

- floribundum. $\mathrm{H}$.

* L hastale, 20.

*__ fallax, 11.

viscosum. F.

*Spielmannia africana, 5. F.

${ }^{*}$ Cyclonema myricoides, 8.

Nuxia verticillata. M.

${ }^{*}$ D Clerodendrum commune, 12. 15.

${ }^{*} \mathrm{E}$ — spicatum, 12.

*F. Lantana Gustavii, 10.

- Sellowii. H. 
Verbenacece-contd.

*Petrrea erecta, 12. 15.

Selagineaces. Elliptic with 3 furrows. (Mohl.)

Selago diffusa. M.

- polygalifolia. $\mathrm{F}$.

fasciculata. P. vii. 14.

rapunculoides. M.

Globularia vulgaris. P. vii. 9.

Stilbe Pinaster. M.

Hebenstreitia dentata. F.

MYoporinex. Elliptic.

Myoporum oppositifolium. P. vii. 6.

ACANTHACEA. According to Mohl, (a) globular or ovoid with spiral bands; (b) globular with reticulated extine; (c) oval reticulate extine; (d) oval or elliptic with long punctate bands (spirally arranged); $(e)$ circular with bands, flat sides; $(f)$ oval with three bands; and, according to Hassall, $(g)$ elliptic, a single slit (Acanthus spinosus).

Thunbergia alata. H. xiv. 47.

* __ fragrans, 16. M. iii. 1.

* - (Meyenia) erecta, 25.

*-_ (Hexacantris) coccinea, 25.

* Sanchesia speciosa, 14. 15.

*Elytraria crenata, 10.

*Gymnostachyum zeylanicum, 10.

* Asystasia capensis, 10.

*Chamæranthemum Bruchii, 12.

*Thyrsanthera stricta, 15.

B Dædalacanthus nervosus, 24.

* macrophyllus, 15.

* purpureus, 18.

Eranthemum pulchellum. H. xiv. 48.

- varians, $\mathrm{F}$.

*- 20.

*Stephanophysium Herbstii, 24.

*Ruellia liliacea, 18.

- formosa. M. 1. 15. M. D. xxxii. 20. F.

*Arrhostoxylum curvifolium, 18.

*Dipterocanthus Herbstii, 16.

${ }^{*} \mathrm{c}$ Barleria argentea, 25. 30.

* - flava, 25 .

*- Gibsoni, 25.

*Cyrthanthera pilosa, 20.

* magnifica, 20. 26.

* _ chrysantha, 20. 25.

*D Justicia carnea, 20.

*— sp. 18. variabilis. H. xiv. 45.

*Dicliptera chinensis, 14. 20.

*Beloperne flava, 20. 24.

*Lankesteria, sp. 25.

*Strobilanthus Wallichii, 26.

* Echmanthera Wallichii, 20.

*Goldfussia isophylla, 18.

* __ anceps, 20.

*__ lanceolata, 18.

*Amphiscopia Pohliana, 16.

Justicia formosa. F. 
Acanthacea-contd.

*Justicia Calitriche, 12. F. Adhadota. F. i. 13.

*Peristropha lanceolaria, 22.

*__ angustifolia, 12. 15.

*Phytoglossa androsæmifolia.

* Stemonacanthus macrophyllus, 25.

*Sericocoma Gheisbeghtiana, 24. WS. 14.

*Libonia floribunda, 20. WS. 13.

*___ plumistigma, 18.

*Asystasia coramandelia, 12.

*Eranthemum tuberculatum, 15.

* - crenulatum, 18.

*Acanthus mollis, 20. P. viii. 13. M. i. 1.

* - montanus, 10.

*Justicia capensis. M. v. 12. Gendarussa. M. purpurea. M.

*Aphelandra fasciculata, 18.

*- nitens, 20.

*__ aurantiaca, 22.

*__ pulcherrima, 12.

*Geisomeria nitida, 24.

Hypoestes verticillaris. M.

$\mathrm{F}^{*}$ Fittonia gigas, 6 .

G Acanthus spinosus. H. xiv. 46. M.

Blepharis capensis. M.

Pedalinez. According to Mohl, round with 12 pleats.

Sesamum orientale. M.

Bignoniaces. According to Mohl, elliptic, with 3 furrows; cellular or parietal.

*Bignonia unguis, 12.

venusta. M. H. 9.

* _ stans, 12.

* _ speciosa, 12.

* _ purpurea, 10.

— Catalpa. P. xx. 15. capensis. F. M.

*Eccremocarpus scaber, 10.

*Tecoma jasminoides, 9. 10. australis. M. F.

*Amphicome Emodi, 15. M. F.

Tanæcium pinnatum. M

Gesneriacex. Elliptic, with 3 slits.

*Gesnera bulbosa, 6.

*__ insignis, 4.

*_- Hontyanii, 6.

- bulbosa. M.

- Douglassii. H. Sceptrum. M.

Gloxinia speciosa. $\mathrm{H}$.

- formosa. M.

- Schotteri. M.

- caulescens. F.

* Echemenes, sp. 6.

*Ramondia pyrenaica, 4. 5. P. ix. 14.

* Sinningia (Stenogaster), 6. 7. Halleri. F. 
Gesneriacea-contd.

*Streptocarpus Kherii, 5. F.

Trevirana coccinea. $\mathrm{H}$.

*Conradia floribunda, 7 .

LENTIBULAREX.

*Utricularia montana, 5. 6.

Pinguicula alpina. M.

OROBANCHEx. Elliptic, with 3 furrows.

Orobanche rubra, 4. 5.

Philippea lutea. M.

ScrophUlarinez. Elliptic, with 3 furrows ; smooth or punctate bands.

*Scrophularia verna, 7 .

- nodosa. H. M.

- aquatica. H.

*Veronica serpyllifolia, 5.

- perfoliata. P. vii. 8.

- longifolia. H. M.

- virginica. $\mathrm{H}$.

- Chamadrys. $\mathrm{H}$.

- hederæfolia. F.

*__ Bauxbaumii, 7. Elongated acutely three-sided. prostrata. F.

*Schizanthus schizophyllus, 9.

— personatus. P.ix. 2.

pinnatus. $H$.

Manulea alternifolia. M.

viscosa. F.

oppositifolia. F.

*Buddleja globosa, 5. H.

Colvillii. Hf. Sik. 17.

spicata. F.

*Calceolaria violacea, 3.

* - scabiosifolia, 5. P. ix. 1.

pinnata. M.

elegans. H.

corymbosa. F.

rugosa. F.

*Rhodophyllum volubile, 7. H.

*Ourisia coccinea, 7.

*Disandra, sp. 7.

*Mitraria coccinea, 9.

*Collinsia grandiflora, 7. F.

*Erinus alpinus, 5.

- lychnoides. F.

*Physocalyx, sp. 8.

Gerardia purpurea. M.

*Russellia juncea, 6. multiflora. M. F.

*Digitalis purpurea. H. WS. 18. Henslow, Cambr. Phil. M.

- Sceptrum. M.

*Tetramera, sp. 6.

*Vandellia crustacea, 5.

Gratiola officinalis. $\mathrm{H}$.

Lindenbergia urticifolia. M.

Alonsoa urticifolia. H. M. F.

Scoparia dulcis. M.

*Antirrhinum majus, 5. H. WS. 19. 
Scrophularinea-contd.

*Antirrhinum Orontium, 6.

- siculum. F.

*Linaria spuria. F.

- pilosa. $\mathrm{H}$.

- genistoides. $\mathbf{H}$.

- dalmatica. $\mathrm{H}$

— purpurea. $\mathrm{H}$.

— triphylla. M.

- Cymbalaria. F.

*Mimulus flavus, 9.

*- Moschatus, 7. M. iii. 3. xx. 29. MD. xxxii. 24. WS. 16.

*__ glutinosus, 10.

* L_ Lewisii. B.

- guttatus. H. xiv. 43.

- roseus. $\mathrm{H}$.

elatus. $\mathrm{H}$.

Chelone barbata. M.

- elegans. M. glabra. M.

*Pentstemon campanulatum, 11. M.

- pubescens. $\mathrm{H}$.

* - pentephyllum, 8. H.

- speciosum. H. F.

- diffusum. $\mathrm{H}$.

ovatum. F.

*Rhinanthus Cristagalli, 8.

*Bartsia odontites, 5 .

* _ viscida, 6.

*Euphrasia officinalis, 8.

*Melampyrum sylvestre, 4 .

*Teedsia lucida, 6 . H. (4 slits).

*Pedicularis sylvestris, 7.

*__ palustris, 10.

* _ megalantha.

flammea. M.

*Celsia cretica, 6 . H.

- lyrata. P. ix. 8.

Arcturus. M.

Angelonia salicariæfolia. F.

Halleria lucida. F.

*Verbascum Thapsus, 10. M.

* Blattara, 6.

phœniceum, 6.

SolANACEx. According to Mohl, elliptic with 3 pleats, or B., with a flattened ellipse, with papillæ or 3 bands. Hassall, cylindric, 2 or 4-lobed. I find them very similar to Scophularineæ, except

Nolana, which is trigonous. Browallia has many forms.

*Browallia elata, 8. 11.

*Streptosolon Jamesonii, 9, 12. 6 or 7 bands.

Anthosercis albicans. $\mathbf{H}$.

- littorea. F. viscosa. F.

Brunsfelsia (Franciscia) Hopeana. H. mutabilis. H. F.

Salpiglossis atropurpurea. H. xiv. 44.

*Solanum Dulcamara, 3. H. WS. 59.

stramonifolium. $\mathrm{H}$. 
Solanacea-contd.

*Solanum nigrum. H. M.

argenteum. P. x. 3. F.

"Solanum tuberosum, 4. H. M.

Lycopersicum erythocarpum. H. M.

*Petunia violacea, 6. H. xiv. 38. 42.

- rosea. $\mathrm{H}$.

atropurpurea. $\mathrm{H}$.

- nyctaginiflora. M.

Hyoscyamus orientalis. P. ix. 15.

canariensis. M. vi. 10.

*__ niger, 8.

pallidus.

physaloides. F.

* Nicotiana rustica, 8. M.

*_ Tabacum. M.

*Datura sanguinea, 10.

- Stramonium. P. xi. 2. H.

Tatula. M.

arborea. F. trigonous.

*Brugmansia, sp. 12.

*Atropos Belladonna, 10. H. 'M.

*Habrothamnus fasciculatus, 12.

Solandra grandiflora. F.

*Lycium americanum, 9.

Africanum. M.

barbatum. M.

Cestrum diurnum. P. x. 3. M. cauliflorum. F.

Saracha viscosa. $\mathrm{H}$.

Physalis oxalidifolia.

Alkekingi. $\mathrm{H}$.

H. pubescens. M.

Capsicum annua. $\mathrm{H}$.

Nicandra physaloides. M.

Nolana paradoxa. H. xvi. 87.

* - prostrata, 11.

Convolvulaces. Mohl, spherical, with a pore with lids. B. echinate with many pores. $\mathrm{C}$. elliptic, with three furrows.

* Calystigia Sepium, 24. H. xvi. 87. M. iv. 10.

${ }^{*}$ Convolvulus arvensis, 20 . H. xvi. 85. WS. 31. M.

- Cantabrica. M.

farinosus. H.

pentanthus. $H$.

*_ microphyllus. M.

- erosus. M. F.

althæoides, 16.

- Dorycnium. M.

— tricolor, 13. M. i. 34.

- Soldanella. WS. 30.

*Batatus triphyllus, 22.

Ipomæa purpurea (major). H. WS. 29.

Sellowii. H. W'S. 33.

Hosfordii. $\mathrm{H}$.

insignis. $\mathrm{H}$.

*Evolvulus arbuscula. 7.

*Cuscuta reflexa, 8. 
Convolvulacea-contd.

*Custuta Epithymum, 4.

trifolia. WS. 32.

Boraginex. Many forms described by Mohl: (a) ovoid, contracted in middle; (b) with papillæ; (c) prismatic with 4 pleats; $(d)$ with 6 pleats; $(e)$ with 10 or 12 pleats. Hassall describes them (a) like Mohl; (b) with tubes opposite to each other with longitudinal fissures; $(c)$ with 10 tubes becoming circular;

$(d)$ four tubes; (e) 3-lobed pyramidal. I have found them usually the first form dumb-bell shaped or with 3 slits.

Borago officinalis. H. xv. 55. M. ii. 28. F. 8 slits.

* orientalis, 6 . F.

*Symphytum officinalis, 7. H. xiv. 53. WS. 9.

* _ caucasicum, 7 .

* Bulla. P. xiv. 19.

*Nonnea flava, 6.

Pulmonaria officinalis. P. vii. 15.

* - saccharata, 6.

*__ angustifolia, 8.

* _ sp., 5.

* _ virginica, 2.

* mollis. M.

*Lithospermum prostratum, 2.

* rubrocæruleum, 3.

* _ arvense, 4.

* Omphalodes verna, 2

*_ linifolia. H. WS. 11.

*Mertensia maritima, 7.

*Onosma echioides, 9. M. v. 9. H.

*Anchusa semperflora, 8.

*__ sp., 9.

- ochroleuca. M. v. 34.

capensis. M. v. 38 .

*Echium Lusitanicum, 12.

* - vulgare, 2.5. M.

- micranthum. P. vii. 13.

- fruticosum. H. M.

- orientale. M.

${ }^{*}$ Lycopsis arvensis, 8. Milleri. M. v. 13.

*Amsinkia, sp. 6.

${ }^{*}$ Myosotis scorpioides, 2. P. vii. 17. M. v. 32. H.

*Cerinthe major. H. Mir. xxxi. 41.

- minor, 6. 8.

- aspera. $\mathrm{H}$.

- bicolor. WS. 10.

Cynnglossum nitidum. P. vii. 18.

pictum, $\mathrm{H}$.

${ }^{*}$ Heliotropium peruvianum, 4. F.

grandiflorum. P. vii. ii. M. ii. 19.

Ehretia lævis. M.

Curdia Myxa. M.

HYDROPHYLLACEX. Hassall describes as cylindric with 3 lobes, or triangular. Those $I$ have examined are in elongated triangular spindles, with a pore on each side.

Phacelia (Eutoca) viscosa. H. xv. 59.

- bipennata. ' $\mathrm{H}$.

- multiflora. $\mathrm{H}$. 
Hydrophyllacee - contd.

Phacelia Wrangelii. H.

Nemophila atomaria, 5 .

* _ insignis, 5. H. xv. 60.

* _ parviflora, 3.

- phacelioides. $\mathrm{H}$.

- pedunculata. F.

*Romanzoffia Sitkinensis, 4.

Wigandia urens. F.

Polemoniacex. (a) According to Mohl, spherical with many pores; punctate. (b) Cellular. ( $c$ ) A flattened ellipse with pore on the equator. I have not seen this form.

*Polemonium cæruleum, 8. H.

*- reptans, 9. P. viii. 2.

Ipomopsis elegans. H. xvi. 83.

*Phlox Drummondi, 10. H. xvi. 82.

— decussata. WS. 58.

*___ divaricata, 5.10.

- undulata. $\mathrm{H}$.

— reptans. P. viii. 3. H.

acuminata. $\mathrm{H}$.

paniculata. $\mathrm{H}$.

*Gilia tricolor, 4. H.

achilleæfolia. $H$.

capitata. H.

*Leptosyphon androsaceus, 11 . H.

- densiflorus. $\mathrm{H}$.

Cantua fotidissima. . P. viii. 4.

pyrifolia. $\mathrm{F}$.

Collomia grandiflora. H. xvii. 80.

rosea. $\mathrm{H}$.

coccinea. $\mathrm{H}$.

lateralis. $\mathrm{H}$.

${ }^{*}$ Cobæa scandens, 25. H. xv. 79. B. cxix. 54 . stipularis. H.

Gentianeacez. Simple ellipse with three slits.

Chironia pubescens, 8.

* - splendens, 10. 12.

Gentiana cruciata. H.

*__ acaulis, 12 . H.

- Amarella. M.

- asclepiadea. M. H.

- concinnis. Hf. Ant. xxxv.

Houstonia coccinea. M. ii. 16.

*Menyanthes trifoliata, 12 . M.

Chlora perfoliata. P. xi. 1.

Swertia perennis. P. xi. 2.

Erythreæa centaurea. M.

Chironia baccifera. M.

- frutescens. M.

Loganiacex. Mohl describes the pollen as ovoid with 3 pleats, but in the only specimen I have examined it was 3-cornered.

Logania longifolia, 6.

- floribunda. F. M.

Gærtnera racemosa. M.

- paniculata. M.

Spigelia marilandica. M.

Apocynaces. Mohl gives 7 forms: $(a)$ ovoid with 3 pleats; 
Apocynacea-contd.

(b) elliptic with 3 pleats and warts; (c) oval with 6 folds; $(d)$ oval with 5 folds with warts; $(e) 4$ warts on the equator; $(f)$ barrel-shaped with a wart at each end; $(g)$ four-fold in the plane.

*Vinca rosea, 16. F. M. iv. 37.

- major. F. M. herbacea. F. P. xi. 4. M. v. 26. H. v. 26.

*Tabernæmontana coriacea, 12. tinctorea. M.

Nerium Oleander. H. xix. 37.

*Dipladenia, sp. 15.

Allamanda cathartica. $\mathrm{H}$.

*__ neriifolia, 12. 15.

*Kopsia, sp. 20.

*Toxicophlæa Thunbergii, 8.

Cerbera fruticosa. F. laurifolia. M. i. 27.

Plumieria phagadænica. M.

*Arduina bispinosa, 7. P. xi. 7 .

*Carissa Carandas, 6.

* - grandiflora, 13.

Echites esculenta. M.

Alyxia aromatica. M.

A pocynum Venetum. M. i. 12. Fourfold.

SALVADORACEA.

Salvadora persica. M.

Oreacez. According to Mohl, elliptic with 3 pleats. Hassall describes it as elongated with 3 lobes.

*Syringa vulgaris. P. xi. 7. F. M.

* persica. F.

*Forsythia suspensa, 10.

*Phillyræa angustifolia. F. M.

- media. M.

Ligustrum vulgare. P. xi. 6. M.

Olea, sp. 8.

- undulata. M.

- verrucosa. M.

- mauritiana. M.

*Fraxinus elatior, 10.

*Jasminum nudiflorum, 10.

— revolutum. F. $\mathrm{H}$.

*__ grandiflorum, 10.

- odoratissimum. M.

- trinerve. M.

- fruticosum. M. azoricum. M. officinale. M.

multiflorum. F.

Sapotaceze. Ovoid with 3 pleats.

Mimosops Elengi. M.

Ebenace.e.

Sideroxylum grandiflorum. M.

Diospyros Ebenum. M.

_- embryopteris. M.

- lanceolata. M.

- discolor. M.

- virginiana. M. 
STYRACEA.

Styrax officinalis. M. P. xi. 9. tomentosa. M.

Symplocos paniculata. M.

Diclanthera laurifolia. M.

Mrrsivex. Minute elliptic with 3 pleats.

*Ardisia polycephala, 5.

- pyramidalis. $\mathrm{M}$.

*Jacquinia, 5.

*Mrsa indica, 3. 4.

* Clavija insignis, 8.

Myrsine variabilis. M.

Primulaces. According to Mohl, elliptic with 3 pleats; according to Hassall, cylindric with 3 bands. I find it either with 3 or with 6 furrows.

*Lysimachia nemorum, 10.

Nummularia. H. M.

* _ thrysiflora, 6.

— vulgaris. P. x. 5. M.

* Anagallis arvensis, 6 . H. M.

*__ tenella, $6 . \mathrm{H}$. collina. M.

${ }^{*}$ Cyclamen persicum, 3. F. hederæfolium. M.

* Androsace Chamæjasme, 4.

*__ acaulis, 2. P. x. 10. coronopifolia. F.

*Dodecatheon Meadii, 2. integrifolium. F.

Hottonia palustris. M.

*Soldanella alpina, 3. 6. F.

*Primula vulgaris, 8. 4. B. cxix. 20.

* - veris, 10. 6. B. cxix. 21. F.

- suaveolens. F.

* - Auricula, 6. M. F.

* _ sinensis, 7. P. xiii. M. F.

* japonica, 7. B. cxix. 28.

* _ cortusoides, 8. F. Trigonous.

* nivea, 7 . n.

* - denticulata, 4 . WS. 8.

* - marginata, 3.

* - alpina, 7. 12.

*__ elatior, 5. 16. M. F.

*__ altaica, 4. 9.

*-_ montana, 4. 10.

*__ purpurea, 3. 8.

* verticellata, 5. 8. F.

*__ sikkimensis, 7 .

— viscosa. WS. 82.

* mollis, 5.

* __ floribunda, 3. 4.

Samolus Valerandi. M.

Plombaginez. Oval to globose, rough, with 3 slits. *Plumbago rosea, 12. P. vi. 1. Lin. iv. 22. F.

* - cærulea, 12. H. xv. 64.

*Armeria vulgaris. H. xv. 61. F. alpina. M. 
Plumbaginea-contd.

*Armeria plantagenia, 14.

- maritima. WS. 85. fasciculata.

*Statice perfoliata, 12.

*__ Halfordii, 12.

— scoparia. H.

- latifolia. M. ii. 10; ix. 19.

tartarica. M. H. 11.

EPACRIDEAs. (a) Fourfold cells, 3 seen at a time; (b) globular echinate or smooth.

Epacris grandiflora. F. M. vi. 10. H. xvi. 91 .

* ovata, 12.

* __ eximia, 11.

— hyacinthiflora. WS. 80.

- paludosa. M.

- ruscifolia. M.

microphylla. M.

Lysinema pungens. M.

Lissanthe subulata. M. paludosa. F.

Andersonia sprengelioides. F.

Acrotriche ovalifolia. F.

Dracophyllum gracile. F.

*Stenanthera pinifolia, 12. 13. 3 or 4 cells.

*Leucopogon, sp. 6. appressus. M. microphyllus. M.

*Styphelia tubifolia, 12.

glauca. M.

Ericaceze. I. Simple ellipse with 3 slits.

II. More usually fourfold, as in Epacrideæ.

I. Blairia ericoides. M. $\mathrm{H}$.

Clethra maderensis. M. v. 16.

*__ arborea, 6. WS. 8. alnifolia. M.

Pyrola secunda. F. M.

rotundifolia. M.

II. Erica multiflora. M. vi. 9.

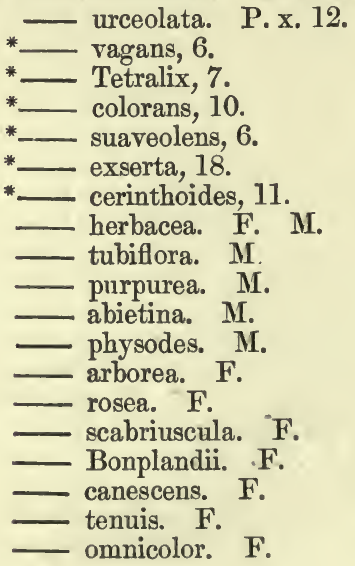


Ericacea-contd.

Erica præcox. F.

*Calluna vulgaris, 8 .

*Azalea indica, 12.

*_- pontica, 12. P. x. 13. viscosa. Mir. $\mathrm{xxx} .48$.

*Rhododendron arboreum, 4. F.

- ponticum. P. x. 14. M. vi. 11.

* Catabwiense. WS. 7.

*__ hirsutum, 10.

*___ Edgworthianum, 15.

*_ Nuttalianum, 10.

*__ Brookianum, 8.

- Caucasicum. H.

Dauricum. F.

Rhodora canadensis. F. M.

Gualtheria procumbens. $\mathrm{H}$.

- Shallon. H.

eriophylla. M.

odorata. M.

*Andromeda multiflora, 7.

- pulverulenta. P. x. 13.

revoluta. M.

salicifolia. M.

longifolia. M.

calyculata. M.

speciosa. F.

*Ledum oppositifolium. 5.

*_ latifolium, 8. M.

palustre. M.

*Dabœecia polifolia, 15.

*Arbutus Andrachme, 10.

*__ Unedo, 10.

*__ integrifolia, 13. canariensis. F.

*Kalmia angustifolia, 8. M. glauca. F.

*Arctostaphylos uva-ursi, 10.

* - tomentosa, 8.

Menziesia Bruchenthallii. M.

ciliaris. M.

cærulea. M.

Salaxis abietina. M. spontane\&. M.

triflora. M.

VACCINIACEX. Fourfold or octohedric.

Agapetis, sp., 15.

Vaccinium Vitis idæa, 10.

*ـ_ 8.

*- serpens. Hf. Sik. 15.

*Macleania speciosa, 15.

*Thibaudia floribunda. 12.

Gaylussacea virgata. M.

retusa. II.

nitida. M.

Camparulacex. Globular echinate or rough or oroid. Lobeleas, minute elliptic with 3 slits.

*Trachelium cæruleum, 5. 6. P. viii. 2. 
Campanulacea-contd.

*Jasione montana, 4.

*Phyteuma orbicularis, 8 . P. viii. 1.

- limonifolia. M. viii. 23 ; ix. 34.

- canescens. M.

*Campanula Medium, 10. M. iii. 18. WS. 28.

*__ Rapunculoides, 8.

- patula. H. xv. 77.

*__ glomerata, 6 .

* — infundibulum. F.

*___ persicifolia, 8 .

- rotundifolia. M.

— nitida. P. viii. 3.

- bononiensis. Mir. xxxi. 43.

— pyramidalis. H. $\mathbf{x v} .76$.

*Specularia spectabilis, 6 .

*Wahlenburghia angustifolia, 6.

*Roella ciliata, 15. Echinate

Codonopsis gracilis. H. Hf. Sik. 16.

*Canarina Campanula, 8. M.

*Lobelia triquetra, 5.

* - spectabilis, 12.

* - syphilitica, 11. M.

- splendens. M.

— trinacoides. $\mathrm{H}$.

— procumbens. H. xv. 78.

- Erinus. H. ignea. $H$. longiflora. M.

Siphocamphylus bicolor. $\mathrm{H}$.

*Centropogon Lucyana, 6. 10.

Goodenovis. Ovate, with 3 bands.

*Goodenia ovata. M. decurrens. M.

Leschenaultia formosa. H. xv. 75. Fourfold.

Scævola lævigata. M. v. 2.

Cyphia bulbosa. M.

Dampiera ovalifolia. M.

STYLIDEXE. ferruginea. $M$.

Stylidum tenuifolium. F. i. 12.

Forstera clavigera. Hf. Ant. 28. (Tri-4gonous.)

Coмposiт止. Oichoneæ. Globular or polyhedric echinate, with 3 to many pores and craters.-Mutisiacea. Elliptic, not echinate, with 3 bands.-Cynarea. Similar to Cichoreæ, or elliptic echinate or smooth. The rest are usually oval or globular echinate, but Artimisia is smooth.

\section{Cichorea.}

*Catananche cærulea, 15.

*Tragopogon pratensis, $18 . \quad$ M. vi. 1 ; xi. 5 .

Cichorea Intibus. M. vi. 7 ; xi. 59 . M. D. xxxii. 27 .

Leontodon hispida. M.

*Taraxacum officinale, 15. P. จ. 10.

Lactuca sativa. M.

*Hieracium Pilosella, 4.

Scolymus hispanicus. M.

* Mulgedium, 10.

*Hypochæris radiata, 10. 
Cichorea-contd.

*Lampsana communis, 9. M.

Scorzonera radiata. P. vi. 11. Lin. iv. 31.

hispanica. H. xv. 67.

Sonchus palustris. M. vi. 5; xi. 61. M. D. xxxii. 28. arvensis. M.

Crepis rubra. M. vi. 6 ; xi. 58. aspera. M.

*Berdanesia rosea, 15.

*Gerbera gossipina, 18.

*Ainslæa aptera, 10.

Cynarea.

*Carduus marianuis, $12 . \mathrm{M}$.

* - palustris, 12.

* _ nutans, 10.

- tenuifolius. $\mathrm{H}$. oleraceus. M.

* Carlina vulgaris, 10.

Carthamus tinctoreus. M.

*Arctium Lappa, 16. Bardana. M.

*Serratula tinctoria, 18.

- xeranthemoides (Cnisus radiatus). P. vii. 17. quinquefolia. M.

*Centaurea nigra, 11. 12.

* Calcitrapa, 8. 10.

*- Scabiosa, 12.

* __ Oyanus, 10. 12. WS. 27. F.

* montana.

* - muricata. M. . 19.

Zoegea Leptaurea. M.

Cynara Scolymus. H.

Echinops sphærocephalus. M. v. 18. lanuginosus. M.

Xeranthemum annuum. M. v. 20. inapertum. M.

Arctodida. cylindricum. M.

*Arctotheca prostrata.

Gazania. WS. 27.

Artolis rosea. M. repens. M.

Cullumia ciliaris. F.

\section{Calendulea.}

*Calendula officinalis, 10. 13. M.

Senecionida.

*Senecio vulgaris, 6 .

* _ aquatilis, 9 .

*__ (Cacalia) splendens, 8. 10.

suaveolens. M.

nemorensis. $\mathrm{H}$.

salicifolja. F.

*(Cineraria) (garden hybrid), 6. F.

lactea. F

palustris. F.

cruenta. F. 
Senecionida-contd.

(Cineraria) maritima. P. xi. 12. Lind. iv. 23. M. i.

*Othonna crassicaulis, 4.

- quinquedentata. $\mathrm{F}$.

*Doronicum Pardalianches, 8.

- orientalis. P. vi. 13.

*Tussilago Farfara, 7. M.

- alba. F.

Anthemidec.

Petasites. F.

*Eriocephalus Africanus, 6.

*Artemisia Absinthium, 7. M. v. 1. Smooth, elliptic.

* _ camphorata, 8.

*__ vulgaris, 9 . M.

* - mutica, 6.

- pontica. M.

Anthemis nobilis. H. Echinate.

*Matricaria inodora, $6 . \mathrm{H}$.

Chrysanthemum viscosa. H. M.

Clusii. P. vii. 12.

*Santolina Chamæcyparissus, 9. M. crispa. M.

*Tanacetum vulgare, 8.

*Achillea Millefolium, 6. M. Ptarmica, 6. 7.

- Eupatorium. M.

Helenacea.

*Tagetes erecta. P. xvii. 10. M.

Brusvillia chrystoma, 6.

Flaveria contrayerba. M.

Helianthea. trinervata. M.

${ }^{*}$ Helianthus annuus, 10. M.

*Galinsoga parviflora, 9. M. trilobata. M.

Melampodum perfoliatum. M.

*Zinnia elegans, 8 . M. multiflora. M.

Dahlia pinnata. P. vi. 16. glabrata.

Cervantesii. WS. 28.

Cosmos bipinnatus. M.

Sanvitalia procumbens. F.

Encelia (Armania) fruticosa. F.

Ambrosia trifida. M. v. 7. artemisiæfolia.

Siegesbeckia orientalis. .H.

Heliopsis pubescens. M.

*Rudbeckia speciosa, 6 . M. purpurea. M.

Madia viscosa. M.

- sativa. M.

Polymnia scabra. M.

Silphium perfoliatum. M. Inulea.

Xanthium orientalis. M. v. 8. Oval, smooth, 3 slits.

${ }^{*}$ Gnaphalium sylvaticum, 6.

- margaritacum. M. 
Inuler-contd.

${ }^{*}$ Helochrysum, 6.

${ }^{*}$ Helipteris Manglisii, 8.

Inula Helenium. M.

Rehlarica pungens. M.

Asterea.

*Bellis perennis, 5 . F.

*Olearia, 5.

*Erigeron canadense, 5. WS. 26.

*

Conyza thrapsoides. M.

squarrosa. M.

*Solidago Virgaurea, 5.

- minuta. P. v. 15.

Chrysocoma coma aurea. H.

Linosyris. M.

Amellus Lynchitis. M.

Aster abbreviatus. M.

- reflexus. F.

- pyrenæus. Mir. xxxi. 33.

Kaulfussia amelloides. M.

Eupatoria.

*Eupatorium cannabinum, 8.

L_ lanceolatum, 6. 8.

glabratum. F.

- purpureum. H.

ageratoides. M.

*Ageratum riparium, 5.

*Stevia serrata, 6. P. xviii. 9.

Vernoniece.

Vernonia montevidensis. M. vi. 12.

DIPSACACEx. (a) Globular, smooth or echinate, with 3 pores. (b) Cylindric, spigot-shaped.

*Scabiosa Columbaria, 18. 24.

- alpina.

* _ succisa, 30.

* _ altipinna, 20.

*__ Fischeri, 50.

- asterocephala (Caucasica). M. iv. 25. atropurpurea. $\mathrm{H}$.

${ }^{*}$ Cephalaria Tatarica, 35.

*Knautia orientalis, 20.

*__ arvensis, 25.

- propontica. M. vi. 36.

Dipsacus Fullonum. H.

- sylvestris. M. iv. 24. WS. 92. inermis, 32.

Morina persicus. M. iii. 16 ; ix. 33.

* _ longifolia, 50.

Valfriaracex. (a) Globular, rough or echinate. (b) Oval in water, trigonous.

*Valeriana rubra, 14 . M.

* - officinalis, 20 . M.

* — Phu, 9. 10.

* - dioica, 12 . F.

— sambucifolia. H. M.

- montana. F.

*Valerianella Lncusta, 10. 
Vulerianacea - contd.

Valerianella olitoria. F.

Robiacex. According to Mohl, 6 forms: (a) Spherical punctate. (b) Ovoid with 3 pleats. (c) Ovoid with more than 3 pleats. (d) Ellipse with 3 pores. (e) Prismatic with 9 pleats. ( $f$ ) $\mathbf{A}$ flattened spheroid with 3 pores. Hassall notes further a fourfold Galea. form, which I also have noted.

Rubia tinctorum. P. vi. 8. M. ii. 26.

*Asperula odorata. $6 . \mathrm{F}$.

*

Crucianella stylosa. H. xv. 70. latifolia. M. ii. 2.

"Sherardia arvensis, 7.

Galium porrigens. $\mathrm{H}$.

* - palustris, 7.

* A Aparine, 5.

* _ saxatile, 4 .

- Mollugo. M. ii. 24.

Spermacocece. cruciatum. F.

Spermacocca verticellata. M. ii. 23.

Crusea strigosa. M. ii. 29.

Anthospermum. M.

Serissa foetida. $\mathrm{H}$.

Opercularia aspera. F.

Paderiea.

Hamiltonia (Spermadictyon) Azurium. M.

Psychotriea.

* Cephaelis Ipecacuanha, 10.

*Rudgia macrophylla, 20.

*Palicourea (Rhodosporum) Gardinerianum, 20.

Morindea. (Nonatellia) triflora. M.

*Morinda, 4.

Ixorea.

*Ixora Bandhuca, 4.

* _ blanda, 4.

- lanceolata. M.

*Pavetta caffra, 6 . H.

Chiococcea. indica. $\mathrm{F}$.

*Chiococca racemosa, 4. 7 .

Gardenea.

Webera pubescens. M.

- corymbosa, 2.

Oxyanthus speciosus. H. xv. 74 .

*Mitriostigma citriodora, 14.

*Randia longissima, 12. 15.

*Gardenia aculeata. F.

* _ globosa, 6.

*Burdellia capensis, 8. H. xv. 73. F.

Fernelia obovata. M.

Hameliea.

${ }^{*}$ Hamelia patens, 6. 7.

- sphærocarpa. M.

* Higginsia microphylla, 4. 
Muscendea.

*Musænda lanceolata, 4. M.

*Coccocypsilum, 8.

Hedyotidece.

Houstonia coccinea. M. ii. 14.

- purpurea. F.

*Pentas carnea, 4.

Rondeletiece.

*Lindenia rivalis, 6.

*Rondeletia, 4.

Cinchonea. speciosa, 4 .

*Luculia, 6.

*Manetta micans, 12.

Danais sulcata. MI.

Coutarea speciosa. M.

Nauclea.

Cephalanthus africanus. M.

CAPRIFOLIACEx. According to Mohl, a flattened ellipse with a papilla at each end; or oval with large pores. I find them spherical echinate with 3 pores, or circular with 8 pores on the circumference. Mr. W. Smith describes them as 3 subtriangular.

*Diervillia rosea, 12.

*Leycesteria formosissima.

*Lonicera Periclymenum, 12. WS. 5. M.

* Tatarica, 13. M. F.

* X.. Xylosteum, 13.

* _ fragrans.

*__ caprifolium. M.

* _ sempervirens. M.

- alpigena. M.

- cærulea. F.

*Linnea borealis, 10.

*Abelia triflora, 16.

*Symphorea racemosa, 8. M. WS. 43.

vulgaris. M.

*Vibernum Tinus, 7 . F. Lantana. M.

Opulus. M.

Sambucus nigra, 10. M.

*_ Ebulus, 9. 4.

racemosa. M.

*Adoxa moschatella, 7. B. cxix. 32 . WS. 6.

\section{CALYCIFLORA.}

CoRnaCEæ. Oval, with 3 bands.

*Cornus mascula. F.

* paniculata, 5.

- canadensis. $H$.

- alba. M.

sanguinea. M.

*Aucuba japonica, 12. B. cxix. 12. Oval 3-lobed. himalaica. Hf. Sikkim, 12. Globular, 3-lobed.

*Corokia buddleoides, 6 . Globular.

Araliacex.

*Aralia ramosa, 10.

sambucifolia. $\mathrm{H}$.

Hedera Helix. H. WS. 75. 
Araliacea-contd.

Panax arborea. M.

- vinosa. M.

Macrocarpus parviflorus. M.

UMBELLIFER A. According to Mohl, cylindric with rounded ends and 3 furrows. According to Hassall, prismatic with constricted sides, and in water with rounded ends and 3 prisms. I find them rather oblong with a constriction in the middle and sometimes a cross-band.

Scandix aromatica. F.

*Chærophyllum sylvestre, 10. B. cxix. 32. F.

* _ tumulum, 6.

Carum Carvi. F. P. xi. 1.

Anethum segetum. Mir. xxxi. 44.

Sium angustifolium. WS. 76.

Heracleum sibiricum. P. xi. 12. Lin. iv. 27.

* - Sphondilium, 6. H. xviii. 146. WS. 63. M.

* _ lanceolatum, 10.

Cachrys seseloides. P. xi. 13.

Eryngium planum. P. xi. 14.

- alpinum. $\mathrm{H}$.

amisthystinum. M.

*Astrantia caucasica. M. v. 1. major, 12.

*Bunium flexuosum, 8.

*Pastinacá sativa, 6.

*Pimpinella Saxifraga, 4. 6.

*Thapsia (melanosilvium) decipiens, 10.

*Did ymus cæruleus, 20. H. xviii. 149.

*Dondia epiphylla, 8. F.

${ }^{*}$ Hacquetia Epipactis, 10.

Conium maculatum. $\mathrm{H}$.

Daucus Carota. $\mathrm{H}$.

Anethum Fœniculum. H.

piperatum. M.

(Enanthe crocata. WS. 69.

fistulosa. $H$.

Bupleurum rotundifolium. H. M.

- fistulosum. $H$.

*Angelica sylvestris, 6. 8. M.

${ }^{*}$ Hydrocotyle javanica, 5 .

- bonariensis, 5 . nitidula.

Ficordese. According to Mohl, ovoid with 3 furrows. According to Hassall, cylindric. I find it so, and also globular, smooth or echinulate.

*Tetragonia extensa, 12.

*Mesembryanthemum spectabile, 6.

*_ aureum, 6 . M.

* _ trigonum, 5.

* - Salmii, 5 .

*__ incurvum, 5.

*__ linguifolium, 6 .

* _ rubricaule, 5 .

* - blandum, 7.

*__ deltoides. P. xiii. 14.

- reflexum. H.

- curvifolium. M. 
CACTACEx. According to Mohl, (a) spherical echinate with 3 bands;

(b) spherical, smooth, with loop form. According to Hassall,

cylindric, 3-lobed. I find them as Mohl describes.

Cactus speciosus. P. xiii. 3.

*Opuntia monacantha, 25.

- polyantha. M. vi. 13.

vulgaris. $\mathrm{H}$.

*Echinocactus Cummingii, 10.

* Mamillaria Gallottii, 10

* - quinquecaulis, 10.

stellata. F.

*Phyllocactus multiflorus, 20.

* Halforsius, 12.

* _ crenatus, 20.

*Epiphyllum Russellii, 13.

*Cereus flagelliformis, 16 . M. ii. 12. F.

*Rhipsalis salicornoides, 10.

* _ sp., 8.

DATISCACE. hexapteris, 11.

Datisca cannabina. P. xviii. 7. M.

Begoniacex. Minute elliptic with slits.

${ }^{*}$ Begonia, 4. nitida. P. xiii. 7.

Cathcartii. Hf. Sik. 13.

Cucurbitaces. According to Mohl, $(a)$ a flattened spheroid with 3 bands and a papilla on each; $(b)$ with a halo; $(c)$ grows in fours, each with 3 pleats; $(d)$ spherical echinate, with 8 to 12 large pores. I also find some trigonous.

*Bryonia dioica, 6. P. viii. 10. B. cxix. 20. L. vii. 35. M.

alba. M.

*Momordica Charantia, 24. H. xvii. 135.

*Trichosanthes Anguina, 15.

${ }^{*}$ Cucumis Melo, 6 . WS. 55. F.

*- sativus, 11. F. M. iii. 22; ix. 32 . WS. 54.

* Colocynthis. M. iv. 15.

* usitatissimus, 15.

Cucurbita Pepo. M. iv. 16; xi. 14. L. vi. Sch. xvi. 20. Mir. xxxi. 47. MD. xxxii. 22. Pol. iii. 47.

*- Melopepo, 30. P. vii. 11.

* - lagenaria, 22 . M. v. 16.

Passiflorex. According to Mohl, (a) elliptic, bent in the middle, with 6 furrows; (b) spherical, rough cellular, 3 or 4 openings with lids.

*Carica Papaya, 11.

*Disemma coccinea, 15.

*Modecca palmata, 15 . With 4 slits.

Melothria fotida. M.

*Passiflora cærulea, 12. H. xvii. 127. MD. xxxii. 23. WS. 53. Mir. xxxi. 46. Poll. iii. 65.

*. capsularis, 12 .

*__ coccinea, $13 . \mathrm{H}$.

*_L Lowii, 20. Sch. xviii. 16.

- Kersemina. F. ii. 13.

- anomala. F. ii. 20.

- racemosa. $F$.

- angustifolia. M. ii. 25.

- aquilegifolia. ND. xxxii. 32. 
Passifloree-contd.

Passiflora penduliflora. M.

triangularis. M.

perfoliata. M.

TuRneracex. Cylindric, 3-lobed, according to Hassall; according to Mohl, ovoid, with 3 pleats.

Turnera elegans. $\mathrm{H}$.

LOASEA. As the above.

*Loasa begoniafolia, 6.

* Laserpitium, 7.

Bartonia aurea. H.

SAMYDACEA.

Humirium (Blackwellia) spiralis. M.

ONAGRACEx. Three-cornered, with a pore at corners-sometimes reduced to two, sometimes fourfold.

Enothera macrocarpa. WS. 1.

- sinuata. $\mathrm{H}$.

— biennis. Sch. xviii. 25. N. ii. 31. L. vi. Mir. xxxi. 29. Pol. i. 20.

- quadrivalvis. $H$.

- suffiruticosa. $\mathrm{H}$.

- Drummondi. H.

Godetia Limbardi. L. v.

rubicunda. H. xviii. 140.

Whitneyii. WS. 2.

Clarkia pulchella. L. vi. WS. 3. Pol. i.

- grandiflora. $H$.

elegans. M. iii. 12 . Sch. xv.

tetragona. P. xiv. 6.

corymbosa. M. iii. 12.

Epilobium roseum. H. xviii. 138. Fourfold.

*- angustifolium, 20. 25. H. xviii. 139. Pol. i.

* montanum, 20. M. vi. 37. MD. xxxii. 14. L. vi. hirsutum. $\mathrm{H}$.

*Circæa lutetiana, 10. M. iii. v. alpina. P. xix. 9. WS. 4.

Fuchsia spectabilis. P. Pol. iii. 60 .

coccinea. P. 5. H. xviii. 142 . Lin. ii. 30.

_ gracilis. $\mathrm{H}$.

- grandiflora. $\mathrm{H}$.

- longipedata. $\mathrm{H}$.

globosa. H.

conica. $\mathrm{H}$.

- lycioides. $\mathrm{H}$.

Devoniensis.

Clintoniensis.

- Atkinsoniensis.

* _ fulgens, 15.

* ___ procumbens, 15.

*__ splendens, 15. H. xviii. 144.

corymbifera. $\mathrm{H}$.

thymifolia, 12. H. xviii. 145.

- microphylla, 10. F.

- cylindrica.

*__ simplicifolia, 15.

Lopezia coronata. $\mathrm{H}$.

- racemosa. H. Mir. xxxi. 40.

miniata. P. xiv. 8. 
Onngracea-contd.

Gaura biennis. $\mathrm{H}$.

Jussiæa erecta. M. iv. 18. Fourfold.

*Trapa natans, 20. P. xiv. 4. Globular, with 3 slits.

LYTHRAREx. According to Mohl, (a) spherical, with 3 bands with a pore and halo; $(b)$ elliptic, with 3 pleats; $(c)$ with 6 pleats and bands.

*Lythrum Salicaria, 3. 5. and 8.5. M. v. 28.

alatum. P. xiv. 10.

"Lagerstæmia indica, 7.8. M. iii. 24.

*Cuphea, sp. 10.

*Nesæa (Hermas) salicifolia, 8. M.

Ammannia sanguinea. M. x. 27.

Duabanga sonnaratoides. Hf. Sik. 11.

Grislea tomentosa. F.

Mefastomaces. Elliptic, with 3 slits, usually very small.

*Melastoma Malabathrica, 12. corymbosa. $\mathrm{H}$. heterophylla. F. H.

*Centranthera inæqualis, 4.

*Medanilla, sp. 3.

*Monochrtum, sp. 6.

*Hexanthus mexicanus, 8.

Arthrostemma lineata. H.

*Sonerila, sp. 4.

Lasiandra oleafolia. M. Funtanesiana. M.

Marestia taxifolia. M. excoriata. M.

Rhexia subtriplicifolia. M.

- contigiosa. M.

Mrrtaces. Trigonous, a pore at each end, except Lecythis and Gustavia.

${ }^{*}$ Calliandra stellata, 4.

*Bæckia virgata, 4.

${ }^{*}$ Callistremon speciosum. F. ruscifolium.

*Callythryx glabra, 4.

*Eugenia Mitchellii, 6.

australis. P. xiv. 14. M. v. 14.

*__ Ugni, 4.

* _ apiculata, 3.

Eucalyptus Eugenioides. M.

- piperata. M.

- paniculata. $\mathrm{F}$. variabilis. F.

Myrtus tomentosa, 6 .
communis, 5. M. iii. 13.

Pimenta. M.

Melaleuca tetragona.

— imbricata.

- fulgens.

- nodosa.

- squarrosa.

- calycina.

- depressa.

canescens. 
Myrtacece-contd.

Melaleuca pulchella.

— thymifolia.

* _ hypericifolia, 5. P. xiv. 12. F. M. iv. 34.

Metrosideros Lophantha. P. xiv. 13.

- pungens. $\mathrm{M}$.

lanceolata. M.

rugulosa. M.

Calothamnus quadrifidus. P. xiv. 16.

villosus. H. xvi. 135.

Angophora racemosa. $H$. cordata. H. xvi. 136.

Tristania variifolia. H. xvi. 137.

Syzygium terebinthum. M.

Leptospermum myrtifolium. M.

- purpurascens. F.

Calyptranthes guineensis. M.

- lucida. M.

Myrcia myoponia. M.

- bracheata. M.

Heyniana. M.

Linkiana. M.

Psidium nigrum. M. rufum. M. cerace. M.

*Darwinia fuschioides, 8.

Gustavia brasilensis. M. 3 pleats.

Lecythis albiflora. M.

- ovalifolia. M.

pedicellata. MI.

Combretack.s. Ovoid, with 3 pleats.

Combretum parviflorum. M.

aculeatum. M.

pulchellum. M.

Quisqualis indica. M.

Poivrea coccinea. M.

Terminalia tomentosa. M. fagifolia. M.

Schousbera coccinea. M.

RHIZOpHoRæ. Yet unknown.

HalagOREAs. Oval, or oval with 3 slits.

*Callitricha aquatica, 6.

*Hippuris vulgaris, 7. F., 4-5 slits, according to him.

*Gunnera scabra, 7.

Myriophyllum spicatum. P. xxiii.

BRUniacex. Oval, with 3 or 6 slits.

Brunia lanuginosa. M.

abrotanifolia. M.

nodiflora. M.

HAMELEMELIDEE. Oval, with 3 slits.

${ }^{*}$ Hamemelis virginica, 6 . M.

Trichacladus crinitus. $\mathrm{H}$.

Droseracese. Globular.

*Drosera rotundifolia, 7. M.

* - anglica, 12.

Crassulacez. Elliptic, with 3 slits.

Crassula lactea. $H$.

- coccinea. H. 
Crassulacea-contd.

Sedum glancum. H. xv. 102.

* - acre, 2. M.

*__ album, 4.

- hispanicum. M.

- spurium. M.

populifolium. M.

*Sempervivum globiflorum, 4. cæspitosum. F.

*Echeveria secunda, $3 . \quad$ IVS. 5.

*Kalanchoe lacinata, 6.

*Cotyledon orbiculata, 10.

aizoon. P. xiii. 15. F.

*Grammanthes chloræflorus, 5 .

Septas capensis. M.

Rochea versicolor. M.

SAxifragacex. Like the above, except the Grossulariæ.

*Saxifraga crassifolia, 4. B. cxix. 29.

- ligulata. F.

- tridactylis. F.

- cuniifolia. F.

- aquatica. P. xii. 18.

- granulata. M.

- cotyledon. M

* umbrosa. WS. 49.

*Astilbe japonica, 3. B. cxix. 38.

Tiarella cordata. P. xii. 17. F.

Mitilla diphylla. F. grandiflora. F.

*Heuchera americana.

*Chrysoplenium oppositifolinm, 6 . alternifolium.

* Parnassia palustris, 7. P. xii. 20.

Francoa racemosa. $\mathrm{H}$.

*Hydrangea hortensis, 6. 7. M. i. 31.

* - japonica, 5.

radiata. M. i. 32.

quercifolia. P. xii. 19.

*Adamia versicolor, 6.

Decumaria sarmentosa. P. xii. 21.

*Deutzia scabra, 3.

* _ corymbosa, 4.

* Philadelphus coronarius. F.

Itea virginica. M. iii. 6.

*Escallonia rubra. F. spectabilis. M.

*Callicoma serratifolia, 3.

*Ceratophyllum gummiferum, 2.

Weinmannia dioica. M.

Bauera rubioides. H. xv. 103.

Ribesea. Spherical.

*Ribes sanguineum, 8.

- frutescens. B. cxix. 39.

- aureum. B. cxix. 36.

- floridum. P. xvii. 4.

*__ Grossularia, 5. 8.

* r rubrum, 9.

Roseacex. Elliptic, with 3 slits, except Poterieæ and Neuradiæ. 
Pyrea.

*Pyrus domestica, 10. P. xiv. 2. M. Malus. F. M.

—_ Cydonia. P. xiv. 3.

* _ japonica. $\mathrm{F}$.

- (Sorbus) hybrida. M.

- $"$ domestica. M.

- $"$ intermedia. M.

- aucuparia. F.

Photinia serrulata. M.

Mespilus Cotoneaster. F.

Cratægus oxyacanthus. F. indica. $B$.

*Rhaphiolepis, 14.

*Chamæmeles, 6.

Neuradece.

Neurada procumbens. M. v. 17. (6 or 8 warts.)

Rosea.

Rosa gallica. M.

- canina. P. xiv. 17.

- bracteata. $\mathrm{H}$.

- lutea. M.

- carolina. M.

Poteriece. semperflorens. F.

${ }^{*}$ Poterium Sanguisorba, 11. M. vi. 24.

* muricatum, 5.

*

*__ annuum, 6.

- verrucosum. P. xiii. 15.

* o officinale, 8.

*__ alpinum, 5. 6.

Agrimonia Eupatorium. H. M.

- himalensis. $H$.

Alchemilla alpina. P. xiii. 6 .

Chamærodes erecta. M.

Quillaija.

Eucryphia Mulligenii. Hf, Tas. vii.

Potentillea.

*Potentilla floribunda, 8.

* _ fragustrum, 8.

- anserina. H. M. F.

-_ aurea. P. xiii. 18.

— atrosanguinea. M. i. 4.

— argentea. M. H.

- tomentosa. M.

- alba. F.

- opaca. F.

- subacaulis. F.

Comarum, 8.

Waldenia geodes. P. xiii. 9 .

Geum rivale. F. M.

- urbanum. M. sinense. $\mathrm{H}$

Dryas octopetala. M.

Rubea.

*Rubus fruticosus, 12. H. 
Rubea - contd.

Rubus odoratus. M.

Spiriea.

*Spirea ariifolia, 7 .

* opulifolia, 7 .

— salicifolia. P. xiv.

- oblongifolia. F.

- grandiflora, 7 .

*_ tilipendula, 5. 6. M.

* Ulmaria. $\mathrm{H}$.

* palmata, 23. Globular.

Pomea.

*Kerria japonica, 6.

*Nuttallia cerasifolia, 8.

*Prunus persica, 11.

— nanum. P. xiv. 15. F.

- Cerasus. F. H.

*__ Padus, 10.

- domestica.

- lenis.

- armeniaca. $\mathrm{H}$.

- avium. F.

Chrysobalanea. maritima. $F$.

Chrysobalanus Icaco. M.

Hirtella paniculata. M.

— hirsuta. M.

LEGUMINOSE. glomerata. $\mathrm{M}$.

Mimosece. A few with a simple grain, with 3 slits; usually compound, 9,12 , or 16 globular or square or drop-shaped.

* Mimosa pudica, 2. R. iv. xxi. 7 .

- agrestis. R. iv. xxi. 8.

- polystachya. P. xvi. 3 .

- mexicana. H. xvi. 104.

- marginata. $\mathrm{H}$.

- laxa. M.

- latispinosa. M.

Schrankia uncinata. R. iv. xxi. 5.

Desmanthus virgata. R. iv. xxi. 1. M. Globular.

- stricta. M. v. 5.

*Albizzia, 12.

*Acacia armata, 10. 12.

* _ retinosa, 6.

* argyrophylla, 10.

* lophantha, 8. P. xiv. 4. M. iii.21. Lin. iv:28. R. iv. xxi. 12.

S. xix. H.

* _ farnesiana, 12.

- mucronata. F. ii. 6.16 cells.

- acapulcensis. F.

- alba. F.

*_L lineata, 8. F. H. xvi. 107.

— floribunda. F.

- stricta. F.

— genistifolia. .F.

- riparia. $\mathrm{F}$.

- longifolia. F.

paradoxa. F. R. iv. xxi. 20. M. 
Leguminosa-contd.

Acacia myrtifolia. F.

undulata. F. R. iv. xxi. 10. H

- pulchella. F.

— decipiens. F. H. xvi. 106. R. iv. 17.

* _ leprosa, 9.

- obliqua.

- alata. R. iv. xxi. 9 .

_ linifolia. R. iv. xxi. 11.

- rutifolia. R. iv. xxi. 13.

- erucifolia. R. iv. xxi. 17.

- arabica. R. iv. xxi. 34.

- verticellata. R. iv. xxi. 38.

- leucocephalus. M. Globular.

*__ Gunneri. Hf. Tas. xrii.

— ovoidea. Hf. Tas. xviii.

- laxa. M. iv. 22.

- chlorantha. M. iv. 21.

— vigens. H. $\mathrm{xv} .105$.

- nigricans. $\mathrm{H}$.

- ciliata. $\mathrm{H}$.

- pulchella. M. $\mathrm{H}$.

- marginata. $\mathbf{H}$.

- trigonocarpa. $\mathrm{H}$.

- sulcata, $\mathrm{H}$.

- fuscifolia. $\mathrm{H}$.

- Julibrissin. M. Globular.

- decurrens. $\mathbf{M}$.

— vera. M.

- compressa. M.

- trichodes. M.

- Lebek. M.

- macrostachya. M.

Inga anomala. M. v. 11. Drop-shaped.

*Calliandra hæmatocephalus, 60. Drop-shaped.

Entada adenanthera. M. Globular.

Adenanthera pavonina. F.

Casalpinea. (a) Globular, echinate, or smooth; (b) three-cornered; (c) oval, with 3 bands.

*Bauhinia aculeata, 28. 30.

- fuscata. M. i. 1.

- armata. M.

- anguina. M.

sp. M. iii. 13.

Poinciana pulcherrima. M. iv. 8. S. xviii. 23. Spherical.

Hymenæa copalifera. M. vi. 23.

- confertiflora. M.

verrucosa. M.

Hæmatoxylon campeachianum. M.

Ceratonica siliqua. M. With 5 pores.

*Oercis Siliquastrum, 4.

Parkinsonia aculeata. M.

Gleditschia horrida. M.

Gymnocladus canadensis. M.

Suella rotundifolia. M.

- poruginea. M.

- paranensis. M.

Codarium nitidum. M. 
Casalpinea-contd.

*Saraca (Jonesia) Asoka, 15.

*Cassia viminea, 8. 10.

* _ occidentalis, 15.

— lævigata. P. xii. 12. M.

trinatitis. M.

- biflora. M.

- marylandica. M.

Papilioniace.

Podalyriece.

Anagyris foetida. M.

Dillwynnia aciculata. M.

Gomphocarpus grandiflorus. M.

*Oxylobium Callistachys, 5.

*Stylophora japonica, 10.

Chorozema Chavii, 6.

*Eutaxia myrtifolia, 8.

Pultenæa paleacea. M.

- protioides. $M$.

deltoides. M. diffusa. Hf. Tas. xiv.

Cyclopia genistoides. M.

Jacksonia scoparia. M.

Podolyria mutabilis. F.

Genistere. styracifolia. F.

Platylobium lanceolatum. F.

Crotalaria angustifolia. F.

Bossia (Scottia) trapeziodes F.

- cordigera. Hf. Tas. xvi.

*Templetonia retusa, 8.

*Goodia lotifolia, 3.

*Lupinus, 12.

- nana. MD. xxxii. 11.

- luteus. $\mathbf{H}$.

- multiflorus. F.

Laburnum vulgaris. WS. 75.

*__ alpinum.

Cytisus foliosus. F. H.

- ramosus. $\mathrm{H}$.

- capitatus. $\mathrm{H}$.

- nigricans. $\mathbf{H}$.

- elongatus. $\mathrm{H}$.

*Genista anglica, 8.

- tinctoria. $\mathrm{H}$.

- sagittata. P. xii. 10.

- canariensis.

- candicans.

— pilosa.

Spartium junceum. P. xii. 18. M.

- scoparium. F. M.

- multiflorum. F.

*Ulex europæus, 6.

Loddegesia oxadilidea. F.

Trifoliea.

*Ononis arvensis, 11. hircinus. $\mathrm{H}$. 
Trifoliea-contd:

Ononis natrix. M.

Trigonella. $\mathrm{H}$.

*Médicago Lupulina, 6.

*__ maculata, 7 .

— arborea. H. F.

- sativa. M.

*Trifolium medium, 10.

- pratense. $\mathrm{H}$.

- _ repens, 10 . F.

*__ suffocatum, 10.

*__ subterraneum, 10.

- montanum. M.

Lotea. rubens. M.

*Lotus corniculatus, 10. H. WS. 74. Gull. Ann, N. H. July 1865.

- major. M. Gull.

Anthyllis polycephalus. $\mathrm{H}$.

Galegea.

*Psoralea macrostachya, 7. 8.

_ pilosiuscula. F.

- bracteosa. P. xii. 7.

- glandulosa. $\mathrm{H}$.

pinnata. M.

Indigofera psoralensis. $\mathrm{H}$.

- madagascarinensis. M.

— tenuifolia. F.

- speciosa. $\mathrm{F}$.

* __ decora, 6. Trigonous.

Galaga officinalis. M.

*Robinia pseudacacia. P. xiv. 18.

- Caragana. P. xiv. 19. F. cæspitosa. P. xiv. 20.

* Clianthus puniceus, 8.

Sutherlandia frutescens. F.

Swainsonia alba. H.

*Colutea frutescens. P. xiv. 1.

- arborescens. H. xvi. 108.

Astragalus Onobrychis. P. xi. 17.

*__ alopecuroides, 5. 8.

- venosus. $\mathrm{H}$.

- tragacanthus. F. monspilanus. F.

Glycirbiza echinata. M.

Hedysarea.

Coronilla Emerus. M.

varia. M.

Hedysarum Onobrychis. M. H.

* _ coronaria, 4. P. xii. 8.

*Stylanthus japonica.

Vicea.

*Arachis hypogea, 10.

*Vicia Faba, 8. $10 . \mathrm{H}$.

* sativa, 9.

- pisifolia. P. xii.

- Cracca.

... sylvatica. 
Vicece-contd.

Vicia hirsuta.

Lathyrus (Orobus) macrorhizus, 6. 10. F.

O. albus. P. xii. 5 .

O. vernus. $\mathrm{H}$.

*- Aphaca, 10.

* odoratus, 10 . M. v. 3.

- latifolius. P. xii. 4.

- pratensis. M.

* __ sylvaticus, 11.

Pisum sativum. M. H.

Phaseolea.

Kennedya rubicunda. P. xii. 6.

- uliginosa. $\mathrm{F}$.

prostrata. F.

monophylla. F.

Erythrina crystagalli. WS. 76. Trigonous.

laurifolia. H. xvi. 109.

Phaseolus coccineus. M.

vulgaris. M.

*Dolichos purpureus, 12.

Cylista tomentosa. M.

Dalbergiea.

Dalbergia Hookeri. M.

Pterocarpus erinacea. $\mathrm{H}$. echinata. $\mathrm{H}$.

Geoffræa mitis. M.

Sophorea.

Virgilia capensis. $\mathrm{H}$.

Sophora racemosa. H.

- japonica. M. (Edwardsia) microphylla. F.

ConNaracese. Oval, with 3 slits.

Onestis glabra. M.

MORINGACEA.

Moringa zelanica. M.

- pterigosperma. M.

AvACARDEACEA. Oval, with 3 slits.

"Rhus Cotinus, 8. M.

- radicans. M.

typhina. P. xi. 16.

Mangifera indica. M.

Anacardium occidentale. M.

Pistacia Terebinthus. M. Spherical.

Lentiscus. M.

Schinus mollis. M.

CorIarIex. According to Mohl, rounded or trigonous.

Coriaria myrtifolia. M.

Sabiacex. Unexamined.

SAPINDACF $\approx$. According to Mohl, (a) trigonous, with a papilla at each corner; (b) spherical or ovoid, with 3 pleats.

Staphylea pinnata. M. F.

trifolia. M.

*Melianthus major, 10. P. xvi. 14. F. M.

*Dodonæa asplenoides, 10.

- viscosa. M.

— triquetra. M.

- angustifolia. F. 
Sapindacere-contd.

*Acer Pseudoplatanus, 15. P. xvi. 14. F. M. platanoides. M.

campestre. M.

- dasycarpum. M.

- monspessulanum. M.

*__ eriophyllum, 8.

* - opulifolium, 12.

Schmidalia senegalensis. $\mathrm{H}$.

Kœlreuteria paniculata. M.

*Xanthoceras sorbifolia, 10.

* Eschylus Hippocastanum, 8. P. xvi. 12. F. M.

*_ rubrus, 10 . F. M.

- macrostachyius. M.

- flavus, 8. M.

Paulinia senagalensis. M.

Cardiospermum Halicacabum. M. iii. 19.

Sapindus emarginatus. M.

Nephelium lappaceum. II.

Euphoria punicea. M.

AMPELIDEE. Elliptic, with 3 pleats, or spherical or slightly trigonous.

Vitis vinifera. P. xi. 15. - M.

*__ quinquefolia, 10.

- Labrusca. M.

- hederacea. M.

*Leea sambucina, 8. M.

Rhamneaces. coccinea, 9.

Rhamnus frangula. P. xi. 12. alaternifolius. F.

Colletia serratifolia. F.

*Trymandrum Billiardii, 4.

*___ sedifolium, 4.

*Pomaderris elliptica, 10. ferruginea. $M$.

*Phylica ericoides, 5 .

- plumosa. F.

*Ceanothus Lobbianus, 7. 8.

- pallidus. $\mathrm{H}$.

Gouania tiliæfolia. M.

*Paliurus Spina Christi, 5.

Cryptrandra Gunneri. Hf. Tas. xi.

STACKOUSIEA.

Stackhousia apiculata. F.

Celastrinez.

*Euonymus europæus, 10.

* - japonicus, 10.

- panus. F.

- latifolius. M.

Celastrus pyracanthus. $\mathrm{H}$.

- scandens. M. oleoides. M.

Cassine peragua. M.

Hippocratea obcordata. M.

ILICINEæ. Oval, rough, with 3 bands.

*Ilex aquifolium, 8. F.

Olacineze.

*Olax Wightianea, 5. 
Olacinece-contd.

*Aptandra Benthamii, 9.

*Chenophora parvifolia, 10.

* _ crassifolia, 11.

*Natsiatum, 4. 5.

*Ximenia americana, 4.

* Strombosia javanica, 9.

*Chaunitia loranthoides, 8.

*Liriosma villosissima, 8.

*Ptychopetalum petiolatum, 4.

Pennantia corymbosa. Hf. N. Z.

Chamliaces. Unexamined.

Meliacex.

*Turræa, 12.

Melia sempervirens. F. Azedarach. P. xviii. 21.

Cedrela odorata. M., with 4 slits.

Busserace.e. Elliptic, with 3 slits.

*Amyris balsamiflora, 4.

OchNacex.

*Gomphia decumbens, 4.

- fimbriata. M.

Ochna atropurpurea. M.

STMARUBRACEE.

Simaba bicolor. M.

*Bucea antidysenterica, 3.

Quassia amara. M.

Aruba acuminata. M.

Balanites ægyptica. M.

RUTACEA. (a) spherical, without pores, or with few pores, or with many Aurantiea. pores, or (b) oval, with 3 broad bands.

${ }^{*}$ Citrus aurantium, 6. P. xviii. 2. F. i. 8 (with 3 to 8 slits). H. xvii. 18.

- Limonium. H.

Cookia punctata. M.

Aglaia odorata. M.

Toddaliea.

*Skimmia obovata, 10. japonica, 10.

Ptelia trifoliata. M.

Toddalia aciculeata. M.

Xanthoxylea. nitida. M.

Esenbachia febrifuga. M.

Xanthoxylum instrumarium. M.

(Fagara) triphylla. M.

Boronicere. fraxinium. M.

*Boronia megastigma, 8.

*__ elatior, 6.

* - tetrandra, 7 .

- pinnata. $\mathrm{F}$.

- Gunnerii. Hf. Tas. $x$.

*Corræa speciosa, 10.

* __ cardinalis. H.

viridis. $\mathrm{F}$.

- alba. F. H 
Boronicea-contd.

*Eriostemon nerioides, 5 .

* myoporioides, 4.

- salicifolium. M. buxifolium. M.

*Acradenia Franklinii, 11.

*Zieria Smithii, 6.

mauritiana. M.

Pobalium truncatum. Hf. Tas. ix.

Diosmieece.

*Adenandra fragrans, 12.

Agathosma obtusa. F. M.

*Diosma ericoides, 11.

* - bucco, 6 .

* _ capitata, 7 .

- imbricata. P. xvii. 7.

- scabra. M.

- pulchella. F.

- alba. F.

- uniflora. F.

Rutea.

Barosma serratifolia. F.

Ruta graveolens. P. xvi. 9. H.

- villosa. M.

- Buxbaumii. M.

Dictamnus Fraxinella. P. xvi. 10.

*

Peganum Harmala. M.

Cuspariea.

*Galapæa Ruldinii, 9.

Spiranthera (Tropanthus) jasminoides. M. Trigonous.

Geraniacese. petiolata. M.

Balsaminea. According to Mohl, cylindric; according to Hassall, 'quadrilateral, with a pore at each end.' I have, however, found them oval, with smooth or rough bands, or sub-globular. I have not seen the pores described by Hassall and Mohl.

*Impatiens noli me tangere, 5. M. iv. 6 ; ix. 36 . H. xvii. 12. MD. xxxii. 21.

* Balsamina, 8. M. iv. 7. Mirb. $\mathrm{xxx} .38$.

*

* __ racemosa, 7. 10. Rough bands.

* royleana, 4 .

OxALIDEA. According to Mohl, elliptic or spherical, with 3 pleats : in - which I agree, some being singularly smooth and shining, others rough ; some elliptic.

*Oxalis Acetosella, 8. WS. 66. I have usually found it globular and shining, but rarely oblong and roughish.

* __ americana, 9 .

* _ corniculata, 6 .

*__ Marlii, 5.8.

* - cernua, 12.

* ___ oregana, 12.

* - carnosa, 10. F.

- Martiana. F.

— floribunda. F. xvii. 7.

- tetraphylla. M. 
Oxalidece-contd.

Oxalis striata. M.

latifolia. M.

- Rheinwardtii. M.

- sensitiva. M.

- eriorhiza. M.

- rostrata. M.

- sulphurea. M.

*__ Plumieri, 10. Oval.

*__ bleupleurifolia, 8.

- crassicaulis. M.

Averrhoa Bilimbi. M.

Vivrarex. Spherical rough.

*Viviana grandiflora, 8.

LimnaNTHE⿸广. Somewhat crescent-shape.

*Limnanthes alba, 4 . Smooth.

* __ pulchella, 5. 6. Smooth.

—- Douglasii. H. xvi. 100. Echinulate.

Pelargontez. Tropæolum, according to Mohl, has a triangular prism ; according to Hassall, cylindric and 3 -lobed. I find T. majus oval, with 3 bands, as represented by Linnæus; nothing like what Mr. W. Smith has figured it as 67 . The T. tricolor is narrow curved, approaching to the Limnanthes. Pelargonium is rough, oval, or globular, almost echinulate.

*Tropæolum majus, 6. 9. P. xvii. 11. Pol. i. 36. Lin. 36. WS.

67. H. Mir. xxxi. 37. Decaisne, Bruxelles, t. xiii. i. 25. peregrinum. $\mathrm{H}$.

*__ tricolor, 6. 8.

*Pelargonium hybridum, 18. 26.

zonale. WS. 61 . F.

*__ lobatum, 18.

*__ capitatum, 20.

*__ ramosum, 12.

*__ uniforme, 16.

*__ fragrans, 11.

- cynosbatifolium. P. xvii. 4.

Geraniacea. peltatum. M.

${ }^{*}$ Erodium cicutarium, 15. M. F.

*Geranium sanguineum, 20. P. xvii. 5. WS. 6.

*__ phlæum, 18.

*__ aconitifolium, 25.

— pratense, 25.

—— sylvaticum. M. vi. $35 . \quad$ H. xvi. 113.

*__ ocellatum, 18.

*__ lucidum.

* - molle, 14. M.

*_ Robertianum, 15. F.

*__ pyrenaicum, 15.

- nodosum. M.

- palustre. F.

ZrgOPHYLLACEx. Ovoid, with 3 pleats.

Nitraria Schoberi. M.

Zygophyllum Fabago. P. vi. 35. H. xvi. 113.

Tribulus. M.

Guaiacum. M.

Malpighraceæ. Mohl describes 11 furms, ovoid, spherical, octahedric, and cubical, with pores in various places. 
Malpighiacea-contd.

*Malpighia punicea, 12 . H. xviii. 116.

*_ sp., 16.

- coccinea. M. vi. 36.

- argentea. M. iv. 4.

Banisteria anguina. M. vi. 27 ; xi. 12.

- glauciphylla. M. versicolor. M. vi. 17.

Aspidospermum macrocarpum. M. vi. 2 ; xi. 9.

Hiræa odorata. M. vi. 22 ; xi. 50.

Gaudichaudia triphylla. M. vi. 18; xi. 53. suffrutosa. M.

Thriallis longifolia. M.

Triopteris brachypteris. M.

Humiriacez. According to Mohl, trigonous.

Humirium floribundum. M. subvaginatum. M.

Lineze. Mohl describes the pollen as ovoid, with 3 pleats; Hassall as square, with 6 tubes at each angle, or cylindric, with 3 lobes: but what I have seen have been spherical, with 3 slits.

Linum usitatissimum. H. xviii. 122.

- africanum.

* - perenne, 10.

* _ grandiflorum, 12. 13.

— trigynum, 8.9. arboreum. P. xvii. 18. Trigonous.

*Radiola Millegrana, 5.

Erythroxylum ferrugineum. M. vi. 20. Globular.

- sideroxyloides. M. laurifolium. M.

Tulaces. Spherical, or ovoid, with 3 slits.

*Tilia europæa, 6. P. xvii. 16. H. xvii. 119. WS. 89.

- americana. $\mathrm{H}$.

- parvifolia. M.

*Sparmannia africana, 8. P. xvii. 15. M. F.

Corchorus tomentosus. M.

Grewia occidentalis. P. xvii. 16. M.

- lanceolata. M. v. 23.

- betulifolia. M.

- ulmifolia. M.

asiatica. M.

Triumphetta major. P. xvii. 4. glandulosa. M.

Entelia arborescens. F.

Eleocarpus speciosus. M. v. 33.

Sloanea paranensis. M.

Lühea paniculata. M.

Sterculactace. According to Mohl, ovoid, with 3 pleats, or globular echinate or with pores at the equator.

Astrapæa Wallichii. M.

Hilsenbergia cannibina. M.

*Dombeya sp., 20.

— pendiluflora. Sch. xvii. 3.

- spectabilis. M.

- elliptica. M.

- acutangula. M.

Pentepetes phonicea. M.

Hugonia Mystax. M. Oval. 
Sterculiacere-contd.

Büttnera heterophylla. M.

Kleinovia Hospita. M.

Pterospernum suberifolium. MI.

Theobroma Cacao. M.

${ }^{*}$ Rulingia corymbosa, 5. 6.

*- parviflora, 5. 6.

*Mahernia violacea, 5.

* - vestita, 2.3.

* - glabrata, 2. 3.

${ }^{*}$ Hermannia angularis, 6.

*__ Althæoides, 5.

* plicata, 6.

- scabra. M. F. 3 or 4 slits.

- hirsuta. F.

- alnifolia. F.

Thomasia purpurea, 4 . solanaceum, 4.

Helicteris hirsuta. M.

Lasiopetalum quercifolium. F. solanaceum. F.

Malvacex. Large, spherical, echinate, with 3 pores.

*Malva rotundifolia, 25.

- sylvestris. H. Sch. xv. 17.

* __ miniata, 25. Mir. xxxi. 31.

- fragrans. $\mathrm{H}$.

- virgata. $\mathrm{H}$.

- mauritiana. F.

nicæensis. $\mathrm{F}$.

limensis. F.

*Althæa rosea, 60 . P. xvii. 13. F. B. cxix. 43. H. L. xv. 14. taurensis. M. iv. 9. officinalis. F.

Lavatera accrifolia. H. xvii. 12. trinervis. S. xvii. 23. Olbia. F.

Malope grandiflora. S. xv. 16.

Kitaibelia vitifolia. $\mathrm{H}, \mathrm{F}$.

Abutilon strictum. H. xvii. 121. Darwinii. WS. 24.

Sida rosea. F. ii. 25.

tiliafolia. F.

vesicaria. $\mathrm{F}$.

*Howittia trilocularida, 25.

Pavonia præmorsa. H.

Malvaviscus (Achania) madagasenensis, 30. F.

Hibiscus liliifolium. H.

— syriacus. H. Mir. xxxi. 35 . Decaisne, Brux. xiii. i. 15. - annuus. $\mathrm{H}$.

rosa sinensis. P. xvii. 2. WS. 23.

— trionum. M. ix. 20 ; ix. 39.

${ }^{*}$ Gossypium herbaceum, 22.

religiosum. L. xvii. 13.

Bombacex. Oval, 3 slits.

Pachira (Carolinea) campestris. M. v. 29 ; ix. 51.

armellaris. M. v. 22.

longiflora. M.

Ochroma Lagopus. M. 
Bombacea-contd.

Bombax pubescens. M.

Gossypium. M.

Wittels' brachia insignis. M.

Eriodendron Samauma. M.

Dipterocarpex. Elliptic, with 3 pleats.

*Shoria robusta, 5 .

Ternstromiaces. Ovoid, with 3 pleats.

*Camellia japonica, 15 . F.

- (Thea) viridis. M. assamia, 7. 11.

Ternstromia. M.

Visnea Mocanera. M.

Caraipa glabrata. M. paniculata. M.

Kielmeyera longifolia. M. coriacea. M.

Ruyschia Bakiana. M.

GUTTIFERx.

Calophyllum Inophyllum. M.

Platonia insignis. M.

Clusia insignis. M.

Monandra gummiflora. M.

HrPERICACEE. Elliptic, or ovoid, with 2, 3, or 4 bands.

${ }^{*}$ Hypericum Androsæmum, 8.

- æoyptiacum. F.

* calycinum, 5. 6.

*__ 4-angulare, 4 . M.

*_ pilosum, 5 .

—_ hircinum. H. xvii. 126.

- perforatum. M. xii. 6.

- canum. P. xxii. 19.

*__ pulchellum, 6.

Vismia baccifera. M.

- guianensis. M.

micrantha. M.

TAMARICACEA. Similar.

*Tamarix gallica, 4.

Portulacace A. Spherical, rough.

*Claytonia carianata, 10. 12.

*virginica, 12. siberica. P. xiii. 1.

*Montia fontana, 3.4. caryophyllia. P. xiii. 8.

*Lewisia brachycalix, 15.

*Canardinia, 11.

Galenia ferruginea. M.

Caryophylutace.s. Subspheric according to Mohl, with 6-12 pores.

I find them usually dodecahedric, except in Spergularia and

Spirgula, which is simple, elliptic, with 3 slits.

*Silene inflata, 8. II.

* maritima, 10 . F.

*_ Armeria, 9. H.

* - vespertina, 16. H. M.

- dichotoma. M. iv. 10.

*__ longifolia, 10.

- pusilla. $\mathrm{F}$.

*Saponaria viscida, 9. H.

- officinalis. H. M. 
Caryophylliacea-contd.

*Saponaria altissima, 8.

- Vaccaria. M.

Gypsophila elegans. H.

* perfoliata, 7 .

*Dianthus barbatus, 8 . H.

* - plumosus, 10.

deltoides, 11.

— Carthusianorum. M.

- caryophyllus, 10. H. x vii. 114.

- annuus. P. xviii. 13.

Agrostemma Githago, 12. L. xvi. WS. 90.

*Lychnis diurna, 10 . M.

* vespertina, 10. B. cxix. 46.

flos cuculi, 16 . $\mathrm{H}$.

- flos jovis. $\mathrm{H}$.

*__ chalcedonica, 10.

*__ japonica, 10.

Alsinea. japonica, 10.
alpina. $\mathrm{F}$.

*Cerastium. P. xviii. 8.

- aquaticum. F. Collinum. F. vulgatum. F.

*Stellaria Holostea, 10. B. cxiv. 48.

*__ graminea, 6. S. xv. 4. F.

- media. F. ii. 1. M.

* pungens. F.

*Arenaria graminea, 7.

* multicaulis, 8.

* _ serpyllifolia, 5. M. balearica. $\mathrm{F}$.

*Sagina procumbens, 4. 5.

*Spergularia rubra, 5. 6.

* pentandra, 6.

*Spergula arvensis, 8-10.

Holostea umbellata. F.

Frankeniex. Elliptic, with 3 slits.

Frankenia hirsuta. M.

- hispida. 'M.

Polygalacez. According to Mohl, spherical, the barrel formed with long pleats; very beautiful under water.

*Polygala vulgaris, 8. WS. 94.

_- spinosa. Lin. iv. 26.

* - bracteosa, 9.

*__ grandiflora, 14. H. xvii. 123.

* C Chamæbuxus, 12. H. M.

* sibirica, 10.

* cordata, 8. F.

*__ myrtifolia. M. v. 30. H.

— latifolia. F. ii. 1.

- speciosa. $\mathrm{H}$.

- Heisteri. F. 8 bands.

- obcordatia. F. 20 bands.

Muraltia filiformis. $H$.

Myta. H.

Monninia Xapalensis. M. v. 31.

*Hymanthera dentata, 5 .

Mundtia spinosa. M. 
Polygalacea-contd.

Corispermum compactum. M.

VOCHYSIACELE.

*Erisma vestita, 4.

*_- Japura, 3. 4.

*Trigonia Lepas, 6. vittosa. M.

- micrantha. M. Spherical, with 8 pores.

- anceps. M.

*Lightea, 4. salicifolia. M.

*Calisthene minor, 5. M.

* Salvertia convallarioides, 10.

*Qualea cordata, 3. 4.

- ecalcarata. M.

Vochysia ferruginea. M.

- pyramilevis. M.

Amphilochia qualioides. M.

Tremandraces. Oval, with 3 bands and pores.

*Tremandra ericifolia, 5.

*Tetratheca verticillata, 7. 3.

Pittosporices. procumbens. Hf. vii.

*Pittosporum undulatum, 8.9. F.

BIXINEA.

"Sollya heterophylla, 8.

Echinocarpus umbellatus. M.

*Cochleospermum Gossypii, 6.

- Ramontii. M.

*Flacourtia sapida, 6.

- cataphracta. M.

Kiggelaria africana. M.

Parkia serrata. M.

Violaria. According to Mohl, (a) elliptic with 3 furrows; $(b)$ flattened, spheroid, triangular, with long pleats; $(c)$ quadrangular or 5-cornered fissures.

*Viola arvensis, 20.25 .

* tricolor, 20. M. vii. 4. H. xvii. 123. B. cxix. 40. WS. 44. F.

*_ cornuta, 15. 8. B. cxix. 42.

* montana. H. xvii. 130.

* - odorata, 12. P. xv.9. M. F. WS. 45 . MD. xxxii. 6.

*— biflora, 9. M.

*__ semperflorens, 11.

- canina. $\mathrm{F}$.

- sylvatica. B. cxix. 19.

- lutea. B. cxix. 41.

hirta. F.

cucullata. F.

Riviniana. F.

Cistrnes. Elliptic with 3 bands.

*Cistus Ladanum.

canadensis. P. xx. 11.

*Helianthemum vulgare, 8.

* - breviatum, 10.

*___ canescens, 12.

${ }^{*}$ Rhodocistus Bertholetii, 12.

Resedacez. Elliptic with 3 pleats. 
Resedacea-contd.

*-Reseda odorata, 12. F. M.

CAPPARIDEA. lutea. M.

Cleome gigantea. M.

- muricata, 7.

- spinosa. $\mathrm{H}$.

- violacea. M.

Capparis ægyptiaca. M.

- cynophodophera. M.

- tomentosa. M.

- spinosa. M.

- Caffra. M.

*Cratæva excelsa, 6.

*Sterophoma cleomiformis, 6.

CroctFara. Ovoid with 3 pleats, cellular or punctate.

Arabidea.

Mathiola incana. $\mathrm{H}$.

*Cheiranthus Cheiri, 9. P. xiv. 4. H. F.

- mutabilis. $\mathrm{H}$.

annuus. $\mathbf{M}$.

*Arabis alpina, 7 . M.

— arabis. F.

- caucasica. F.

Cardata. F.

*Cardamine digitata, 6. 7 .

- pentaphylla. F.

- hirsuta. F.

- pratensis. F.

Alyssinece.

*Aubretia deltoides, 5. 6. H. B. cxix. 12. F.

*Alyssum saxatile, 6 . F.

— podolicum. P. xiv. 9.

- rostratum. M.

- incanum. M.

- saxatile. $M$.

*Draba, 6. montanum. M.

Cochlearia officinalis. M. F.

Pringlia antiscorbutica. B. cxix. 56.

Sisymbrea.

"Schizopetalum Walkeri, 6.

*Sisymbrium officinale. B. cxix. 57.

* L Irio, 6.

angustifolium. M.

*Pachypodium, 7.

Heliophila araboides. H.

Brassicee.

Hesperis matronalis. M.

*Brassica oleracea, 8. H. xvii. 130. M.

- Napus. Ḿ.

- (Sinapis) alba. H. M.

* nigra, 8.

*Diplotaxis muralis, 10.

*Villa pseudocytisus, 7. 8. F.

*Orychophragma sonchifolia, 6 . 
Lepidea.

*Athionema orientalis, 3. 5.

*Lepidium sativum, 4.

*Capsella bursa pastoris, 3.

Thlaspidea.

*Iberis odorata, 7. 8. M.

* _ sp.

3. 4.

- umbellata. M.

- sempervirens. M. F.

Hutchinsia australis. Hf. iv.

Thlaspi arvense. M.

Isatidece. perfoliatum. M.

Peltaria alliacea. M.

Bunias orientalis. P. xiv. 1.

Cakilea.

Myagrum paniculatum. M.

* Cakala maritima, 11.

Crambe grandiflora. P. xiv. 17.

Raphanea. cordifolia. M.

"Raphanus sativus, 9. M.

- Raphanistum. H.

caudatus. P. 18.

Papaveraces. According to Mohl, Papaverece ovoid or spherical, with 3 bande, narrower or punctuate; Fumariea, spherical, with 6-8 papillæ, or with 3 bands, with triangular or cubical prisms or dodecahedrous. Hassall gives Papavereæ cylindric with 3 tubes, or spherical with 6 fissures; the Fumarieæ spheroidal with 6 apertures. I find them as described by $\mathrm{Mohl}$; but the Fumaria officinalis I should call more cubical, with a large pore on each

Fumariea. side.

*Fumaria officinalis. H. xxiii. 153. WS. 81. spicata. M. ii. 36.

* Dielytra formosa, 8. P. xiv. 22. H.

* canadensis, 7 . P.

*Corydalis lutea, 9. P. xir. 6.

* _ـ solida, 8 .

* _ bulbosa, 9 .

* cava, 9.

* _ capnoides, 10.

*__ claviculata, 8.

Papaverea.

*Eschscholtzia californica. H. xvii. 154.

*Chelidouium, 5.

— majus, 8. M. H. P. xv. 6. F.

Glaucium luteum. P. xv. 7.

*Bocconia cordata, 4.

*Sanguinaria, 15.

*Meconopsis cambrica, 4. 5.

- simplicifolia. Hf. Sik. 8.

— nepalensis. Hf. Sik. 9.

*Argemone mexicana, 8.

* Papaver somniferum, 10.

* bracteatum, 9.

- orientale. P.xv. 3. M.

*__ Rhœas, 4. 5. M. WS. 88. 
Papaverea-contd.

*Paparer dubium, 8.

* pilosum, 6.9.

aurantiacum. F.

*Platyloma californica.

Sarracteviacese. Ovoid, with 8 bands with pores.

*Sarraeniia flava, 7.

* - purpurea, 6.

*_ (hybrid), 7.

NrmpHeacese. Ovoid, with a single slit, either echinate or smooth.

*Nelumbium speciosum, 15. Echinate.

*Nuphar luteum, 15.

* Nymphra alba, 9.

* stellata, 12. Smooth.

*- Lotus, 10. M. i. 29.

*__ purpurea, 8.

*- pubescens, 5 .

BERBERIDACEA. Globular.

Berberidece.

*Berberis Aquifolium, 9. F. 22.

- - japonica, 9.

* _ dulcis. 10.

* _ asiatica, 10.

* Darwinii, 12.

* vulgaris, 9 . F.

*Epimedium alpinum, 10. H. M.

*- pinnatum, 10.

- - grandiflorum, 10.

Leontice Leontopetalum. M.

- altaica. F.

*Bongardia Rowalfii, 13.

*Diphylleia cymosa, 7.

*Jeffersonia diphylla. P. xv. 4.

Podophyllum peltatum. P. xv. 5.

*Aceranthus diphyllus, 9.

Lardizabalea.

*Akebia, 6.

*Holbollia latifolia, 4. 5.

Decaisnea insignis. Hf. Sik. 10.

Menispermaceæ.

*Stephania, 11.

Menispermum canadense. M.

Cissampelos orbiculatus. M.

Arroracem.

*Meliusa, 10.

Magnoliace.e.

Schizandrea.

*Schizandra, 6.

Winterece.

*Drymis Winteri, 9.

* arumatica, 7 .

*Ilicium anisetum, 6.

* _ floridum, 7.

Magrolines. Simple, elliptic, with 1 slit.

* Magnolia, 8.

*-Yulan.

*__ furcata, 10. 
Magnoliea-contd.

Magnolia grandiflora. H. xviii. 150.

- glauca. $\mathrm{H}$.

Michelia Cathcartii. Hf. Sik. 6.

Talauma Hodgsonii. Hf. Sik. 6.

Liriodendrum Tulipifera. P. xvi. 5.

DILLENICEA.

${ }^{*}$ Hibbertia dentata, 8.

*__ ericifolia, 6. Hf. Tas. iii.

- crenata. P. xvi. 4.

* _ Rheadii, 8. P.

* - saligna. M.

- canescens. M.

- grossulariæfolia. F.

Candollea cuneiformis. H.

Delima. M.

Curatella. M.

Tetracera. M.

RaNUNCULACEA.

Proniece. Ovoid, with 3 slits; large.

*Pæonia coralina, 20.

* montana, 10 . F.

- renifolia. P. xvi. 3.

Helleborea. Ovoid, with 3 slits; smooth or punctate.

*Aconitum Napellus, 4. 7.

- Lycoctonum. P. xvi. 13.

Delphinium Staphysagria. II. M.

- Reginæ. P. xv. 14.

*__ elatum, 8.

- Ajacis. M.

- Consolida. M.

- tricorne. F.

*Aquilegia vulgaris, 7.

Gibsoni. P. xx. 16.

Nigella damascina. MI. ii. 2.

*Isopyrum thalictroides, 6 . F.

Eranthis hyemalis. F.

*Helleborus niger, 8. 9. F.

*__ lividus, 7. 8.

fuscus. P. xvi. 2.

fotidus. H. M. F.

purpurascens. F. viridis. F.

*Trollius europæus, 5. M. F.

Caltha palustris. P. xvi. 1. M. F.

Ranunculea. (a) Globular echinate; (b) ovoid, with 3 slits and bands.

*Ranunculus arvensis, 15.

* - muricatus, 8.

* - montanus, 6.

* L lomatocarpus, 8.

*__ pyrenaicus, 6 .

*- auricomus, 7.8. F. (4 slits.)

* - bulbosus, 7 .

* _ acris, 6. 11. H. xviii. 156. M. ii. 7.

*__ repens, 5.

* _ parvifolius, 8.

* - Ficaria, 8. B. cxix. 11. F.

*- asiaticus, 8. Dodecahedric. 
Ranunculere - contd.

*Ranunculus giganteus, 12.

- Philonotis. F.

* - flammula, 4. 5 .

*__ Lingua, 8. Spherical.

* pumilus, 5 . F.

*__ aconitifolius, 6.

* - hederacea, 8.

Anemonea. aquatilis. $\mathrm{F}$.

Adonis æstivalis. M. i. 25.

*__ vernalis. P. xii. 12. F. pyrenaica.

*Anemone Pulsatilla, 8. P. xv. 13.

*__ Coronaria, 8.9.

* - Pavonina, 9. 10.

* L hortensis, 8. F.

*__ vitifolia, 12.

*__ vernalis, 10.

* alpina, 6.

*__ pulchella, 9. 10.

*__ blanda, 8.

* Halleri, 11.

*__ arborea, F.

* a _ apennina, 6. 7. F.

* nemorosa, 6 . F.

*__ trifoliata, 8. 9.

*__ canadensis, 8.

*- Hepatica, 10. F.

*__ polyantha, 5 .

* —_ narcissiflora, 8.

*_- sylvestris, 3. 5.

*Thalictrum majus, 4.

*__ flavum, 4. 5.

Clematidere. glaucum, 4. 6.

Atragene austriaca, 7. alpina. M.

*Clematis montana, 7.

* r reticulata, 2. 3.

* _ cærulea, 5 .

*__ flammula, 7. 10.

*__ Buchaniana, 4. 5.

*__ vitifolia, 5. 6.

*—_ orientalis, 5. 6.

*__ Jackmanii, 6. 7.

* - erecta. M.

* _ angustifolia. M.

* linearis. Hf. Tas. i. 



\section{EXPLANATION OF THE PLATES.}

\section{Graminex.}

1. Zea Mays $\left(\frac{20-18}{6000}\right): a$. a beautiful topaz colour, transparent, prismatic, with 4 sides, the top larger than the bottom. $b$. from the top looking through the prism.

2. Triticum sativum $\left(\frac{20}{6000}\right) ; 6-5-$ sided, with hollows in each side and the ends.

3. Alopecurus pratensis $\left(\frac{10}{6000}\right)$ : a. similar, but narrower. $b$. the bottom. c. the top.

4. Festuca ovina $\left(\frac{7-6}{6000}\right)$. a. similar. b. top.

5. Panicum plicatum $\left(\frac{10}{6000}\right)$; nearly globular, with three pleats, and hollows under each pleat. $b$. under water clouded.

\section{Cyperacex.}

6. Carex panicea $\left(\frac{7}{60 v 0}\right)$ : a. 4-sided, with hollows on each side. b. pyramidal. c. oval; ovalclouded.

7. C. arenaria $\left(\frac{8-10}{6000}\right): a$. an elongated pyramid. b. parallel sides, with pores in each side. c. withered form.

8. Cyperus alternatus $\left(\frac{5-6}{6000}\right): a$. 3-cornered. $b$. looking down.

\section{Pontederache.}

8. Pontederia dilatata, Bus. $\left(\frac{9}{6000}\right)$; eje-shaped, with a single pleat, somewhat rough numerous raphides in the anther cell.

\section{JUNCACEA.}

10. Juncus effusus, $L .\left(\frac{6}{6000}\right): a .4-$ 6-sided, prismatic, smaller at ends, with hollows on each side. $b$. swelling in oil. c. globularclouded.

11. Xerotes longifolia $\left(\frac{5}{6000}\right): a .3-$ cornered. b. c. in vinegar rough and globular.

\section{Asparagex.}

12. Dianella strumosa $\left(\frac{4}{6000}\right): a$. 3cornered. $b$. in oil.

13. Asparagus officinalis $\left(\frac{8}{6000}\right): a$. eye-shaped. $b$. in oil. $c$. in vinegar. $d$. in water globular oil-diffused.

\section{Astalez.}

14. Astelia Cunnaghamii, Hook. $\left(\frac{6}{6000}\right): a$. rough with 1 slit. $b$. in oil. $c$. in vinegar. $d$. in water.

\section{Smitace de.}

15. Philesia buxifolia, Lam. $\left(\frac{15}{6000}\right)$ echinulate globular; no pores found; no change in oil or water.

16. Trillium erectum $\left(\frac{8}{6000}\right)$ : a. oval or nearly globular-opaque. b. c. under oil four or five facets with a pore on each side. $d$. in water globular clouded; with large raphides in the anther cell. 


\section{LILIACEA.}

17. Lilium auratum $\left(\frac{30}{6000}\right)$ : a. brilliant orange red; a strong network. $b$. in oil a long slit; oil diffused in the olive oil.

18. Brodiea sp. $\left(\frac{15}{6000}\right): a$. yellow, punctured with green spots. b. gibbous.

19. Tulipa Gesnerii, L. $\left(\frac{15}{6000}\right)$ : $a$. oval, with a single slit, yellow; in oil opening out with 2 or 3 foids, or $c$. $d$. opening in a straw-hat shape.

20. Scilla nutans $\left(\frac{15}{6000}\right)$ : a. elliptic, with 1 slit. $b$. oval.

\section{Agavex.}

21. Bischemoria Tonelli $\left(\frac{25}{6000}\right)$; in 4 cells, and the rich outer coat of lacework.

\section{Asparaginez.}

22. Stylandra pulchella $\left(\frac{6}{6000}\right)$; 3cornered, pyramidal.

23. Drimyopsis Kirkii $\left(\frac{10-12}{6000}\right): a$. crystalline like a tooth. $b . c$. in oil showing pores at each side. d. oval clouded.

\section{AroIdexe.}

24. Anthurium Hookeri, Kth. $\left(\frac{3}{6000}\right)$ : a. rough, globular; pore discovered in water. $b$. almost transparent, numerous minute raphids in the anther cell.

25. A. cordata $\left(\frac{3}{6000}\right)$; globular, rough.

\section{TrpHaces.}

26. Typha latifolia, L. $\left(\frac{9}{6000}\right)$; subglobular, with 4 valves, which open out under oil or water; numerous raphids in the anther.

\section{Palmace.}

27. Areca Baueri $\left(\frac{12}{6000}\right)$ : a. transparent, eye-shaped, smooth. $b$. in water oval, clouded.

\section{Aponogetonex.}

28. Aponogeton distachyum $\left(\frac{6}{6000}\right)$ : $a$. elliptic, minutely punctulate, 1 slit. $b$. in oil. c. in water globular, clouded.

\section{Filuviales.}

29. Potomogeton crispus, L. $\left(\frac{5}{6000}\right)$ : $a$. a simple sack, rough. $b$. in water globular and somewhat echinulate.

\section{JUNCAGINEA.}

30. Triglochin palustris, L. $\left(\frac{4}{6000}\right): a$. eye-shaped, 1opening with roughnesses. $b$. globular clouded.

\section{Arismacem.}

31. Alisma Plantago $\left(\frac{6}{6000}\right)$; polyhedric, pores in each facet.

\section{Butomex.}

32. Hydrocleis Humboldtii $\left(\frac{7-8}{6000}\right)$ : $a$. echinulate, globose. $b$. the prickles disappear under oil, when 3 pores become visible. c. clouded in water.

\section{DIOSCORIDEX.}

33. Tamus communis, $L \cdot\left(\frac{10}{6000}\right): \quad a$. oval, with a strong band, pitted. $b$. in water globular, clouded, numerous raphids in the anther cell. 


\section{AMARYLLIDACEX.}

34. Puncratium caribcen $\left(\frac{25}{6000}\right): a$. a long ellipse; the extine covered with yellow matter; thickened at the ends. $b$. under oil the oily matter is diffused and the network becomes visible. $c$. in water it contracts in length and has the oily particles in drops.

35. Alstrameria curtisiana $\left(\frac{10}{6000}\right): a$. yellow, in natural state opaque. $b$. with delicate markings; pale lilac, and with pale yellow markings, with a long slit. $c$. in vinegar oval, finely marked; in water nearly globular, with circular rim of oily blots all round.

36. Amaryllis formosissima $\left(\frac{25}{6000}\right): a$. 1 slit; the extine punctulate. $b$. oval in water.

37. Cirinum sp. $\left(\frac{16}{6000}\right)$ : a. yellow, punctulate. $b$. with 1 slit with 2 coverings. c. in water oval.

\section{IRIDEA.}

38. Crocus syriacus $\left(\frac{15-20}{6000}\right)$; smooth, globular.

39. Schizostylis coccinea $\left(\frac{15}{6000}\right)$; with a single pleat, with minute lines on the coat.

40. Gladiolus (hybrid) $\left(\frac{20}{6000}\right)$; extine pitted, with a long slit; in water globular.

41. Iris sinensis $\left(\frac{12}{6000}\right): a$. rough, broad, oval. $b$. in oil with long slit; in water globular and clouded.

42. Iris elatior $\left(\frac{25-30}{6000}\right)$; reticulate, 1 slit.
TACCACEE.

43. Tacca altocarpifolia $\left(\frac{14}{6000}\right)$; hyaline, oval, with 1 simple opening.

44. Ataccia cristata $\left(\frac{12-18}{6000}\right)$ : a. yellow, 1 slit. $\quad b$. in oil 1 or 2 folds on the covering, which is delicately marked.

45. Musa paradisiaca $\left(\frac{20}{6000}\right)$; broad, oval, with rough lines; unaltered in water.

46. Canna indica $\left(\frac{16}{6000}\right)$; globular, rough, almost echinate; unaltered in water.

\section{DICOTOLYDONEAE.}

\section{Crcadiex.}

47. Ceratozamia mexicana, Bronv. $\left(\frac{10}{6000}\right): a$. transparent, oval, with 3 slits. $b$. in oil. c. in vinegar. $d$. in water globular and clouded.

\section{CoNifere.}

48. Cryptomeria japonica, Don. $\left(\frac{4}{6000}\right): a$. sub-spherical, sometimes with a little projection. $b$. in oil somewhat cubical. $c$. in vinegar with turbid fovilla. $d$. and $e_{\text {. }}$ in water bursting with a cloud of oily particles mixed with fovilla through a crescentshaped opening.

49. Juniperus communis, L. $\left(\frac{3}{6000}\right)$ : a. sub-spherical, with 4 or 5 valves apparently. $b$. in oil 4-5 valves, transparent. $c$. in vinegar spherical. $d$. in water.

50. Cupressus Benthami, Eud. $\left(\frac{5}{6000}\right)$ : a. 3-4-sided. b. c. in oil. d. in water.

51. Pinus laricio, Poir. $\left(\frac{15}{6000}\right):$ a. 2 oval masses full of greenish-yel- 
low fovillæ, connected by a thin circular plate. $b$. somewhat opening out in oil ; unaltered in water.

52. Larix communis, $L .\left(\frac{8}{6000}\right)$ : a. globular rough, with 3 pores. $b$. in oil more transparent. $c$. in vinegar with oily blots diffused; in water globular and clouded.

\section{Corylaceac.}

53. Corylus avellana, $L .\left(\frac{6}{6000}\right): a$. when fresh globular, but rapidly becoming polyhedric, with pentagonal facets with a pore in each. $b$. in vinegar. $c$. in oil. $d$. in water.

54. Carpinus Betulus, L. $\left(\frac{5}{6000}\right): a$. opaque, globular. $b$. with 3-loop openings, with a pore in each.

\section{OUPULIFERA.}

55. Quercus cerris, L. $\left(\frac{10}{6000}\right): a$. oval, with 3 bands and slits, the bands minutely fitted. $b$. in vinegar somewhat 3-cornered. $c$. in water subspherical, with the remains of 3 pores.

56. Fagus sylvatica, $L .\left(\frac{7-8}{6000}\right): a$. broad, oval, with 3 slits. $b$. seen endwise.

\section{Crtinace不.}

57. Cytinus hypocystis, L. $\left(\frac{4}{6000}\right)$ : $a$. oval, with a pore. b. united in 4 pairs. c. globular in water.

\section{Aristolochiachis.}

58. Aristolochia clematitis, L. $\left(\frac{8}{6000}\right)$ : $a$. opaque, rough globular. $b .2$ bands visible in oil. $c$. in vinegar.
59. Aristolochia brasiliensis, Matt. and $Z$. $\left(\frac{3-10}{6000}\right): a$. globular rough. $b$. in vinegar with delicate workings on the edge.

\section{Euphorbiaceza.}

60. Euphorbia bupleurifolia, Jou. $\left(\frac{14}{6000}\right)$; shining, yellow, elliptic, with 3 slits; in water globular clouded.

61. Poinsettia pulcherrima $\left(\frac{10}{6000}\right)$; oval, rough, with 3 slits; in oil, with a margin from the roughnesses.

62. Mercurialis perennis, $L$. $\left(\frac{7-8}{6000}\right)$ : a. smooth, elliptic, with 3 slits. $b$. in oil. $c$. end up.

63. Croton humitis, L. $\left(\frac{18}{6000}\right)$; globular, rough, opaque, with 3 slits.

\section{SALICINEX.}

64. Salix pentandra, $L \cdot\left(\frac{6}{6000}\right): a$. oval, with 3 rough bands. $b$. end up. $c$. in oil. $d$. in water 3 bands still visible.

\section{Platanaceze.}

65. Platanus orientalis, L. $\left(\frac{2}{6000}\right)$ : $a$. very minute, oval, opaque. $b$. in oil, with 3 bands. c. in water spherical.

\section{Betulacez.}

66. Alnus glutinosa, $L \cdot\left(\frac{6}{6000}\right)$ : $a$. opaque, polyhedric. $b$. in water sub-globular.

\section{ULMACEAE。}

67. Ulmus campestris, L. $\left(\frac{10}{6000}\right)$; globular, rough, with curved 
markings. $a . b . c$. opening at one side. $d$. in water. $e$. in vinegar, 5-cornered, polygonal.

\section{Cannabidea.}

68. Cannabrs sativa, $L .\left(\frac{7-8}{6000}\right)$; oval, transparent, with 3 slits; globular in water.

\section{Moracee.}

69. Dorstenia maculata, Lam. $\left(\frac{4}{6000}\right)$; polyhedric, 5 sides to each side of each facet; in water subglobular, with many facets.

\section{URTICACEA.}

70. Parietaria officinalis, $L .\left(\frac{3}{6000}\right)$; globular, reticulate.

\section{Proteacex.}

71. Grevillia macrostylis $\left(\frac{15}{6000}\right)$.

72. Grevillia lanceolata $\left(\frac{15}{6000}\right) ; 3-$ cornered, but little altered under oil, vinegar or water.

73. Banksia integrifolia $\left(\frac{6}{6000}\right)$; with but 2 pores.

\section{Eleagraces.}

74. Eleagnus hortensis, M.B. $\left(\frac{15}{6000}\right)$; opaque, with 4 slits (in oil); under water spherical, clouded.

\section{THYMELEE.}

75. Daphne odora, Thu. $\left(\frac{5}{6000}\right)$; opaque, globular; under oil rough, with a margin; in vinegar almost clear, with a margin.

\section{LAURINEA.}

76. Laurus nobilis, L. $\left(\frac{7}{6000}\right)$; globular, rough; under water with a margin.

\section{ParanYChIEe.}

77. Corriogiola littoralis, L. $\left(\frac{2}{6000}\right)$; minute, sub-spherical.

\section{Chenopodiacez.}

78. Chenopodium Bonus Henricus, L. $\left(\frac{8}{6000}\right)$; sub-spherical, punctate; in water, with numerous blots of oil protruding.

\section{Amaranthez.}

79. Amaranthus tristis, L. $\left(\frac{6}{6000}\right)$; sub-spherical, with numerous facets.

80. Celosia cristata, Moq. $\left(\frac{5}{6000}\right)$; polyhedric, with pentagonal facets; in oil, with distinct echination.

81. Avra sanguilenta $\left(\frac{4}{6000}\right)$; polyhedric, with 5 pentagonal facets; in oil a pore visible to each facet.

\section{Polygonaces.}

82. Rheum officinale $\left(\frac{8}{6000}\right)$; oval, rough, with 3 bands and slits.

83. Rheum undulatum $\left(\frac{8}{6000}\right)$ : a. elliptic and smooth, with 3 slits. $b$. in oil more clear. $c$. endwise. $d$. in water spherical, clouded.

84. Polygonum Bistorta, L. $\left(\frac{12-16}{6000}\right)$; rough, oval, with 3 slits; in water spherical, clouded.

85. Polygonum hydropiper, $L$. $\left(\frac{10}{6000}\right)$ spherical, echinulate; unaltered in water.

86. Rumex obtusifolium, L. $\left(\frac{8}{6000}\right)$; somewhat 4-sided, with 4 pores; in water globular, clouded. 


\section{Phytolaccaceas.}

87. Rivina humitis, L. $\left(\frac{6}{6000}\right)$; globular, with 3 pores.

88. Rivina lacvis, L. $\left(\frac{7}{6000}\right)$; oval, with small facets.

\section{Nyctaginez.}

89. Fisonia olfersiana, Lamk: $\left(\frac{8}{6000}\right)$; opaque, spherical, unaltered in water; numerous raphides in the anthers.

90. Bougainvillea spectabilis $\left(\frac{8}{6000}\right)$; polyhedric, a pore in each facet.

\section{MONOPETALAE.}

\section{Plantagineze.}

91. Plantago media, $L .\left(\frac{5}{6000}\right): a$. sub-spherical, opaque. b. $c$. in oil, polyhedric, with many pentagonal facets, with a pore in each. d. in water, spherical, clouded.

92. Littorella lacustris $\left(\frac{4}{6000}\right): a$. sub-spherical. $b . c$. in oil, with punctate bands. $d$. globularclouded.

\section{Labiata.}

93. Scutellaria coccinea, $H$. $B$. and K. $\left(\frac{10}{6000}\right)$; oval, punctate, with 3 bands and slits.

94. Ajuga reptans, L. $\left(\frac{9}{6000}\right)$; simple, oval, with 3 bands and slits; in water globular clouded.

95. Ocymum sanctum, L. $\left(\frac{8}{6000}\right)$; oval, with 6 bands and slits; in water globular, the bands barely visible.

96. Salvia ccerulea $\left(\frac{10}{6000}\right): a$ oval, with 6 bands and slits. $b$. the end upwards. $c$. in water globular, with drops of oil protruding.

97. Plectranthus australis, Brown. $\left(\begin{array}{c}10 \\ 6000\end{array}\right) ; 3$ rough bands and 3 slits.

98. Prostanthera nivea, Gesn. $\left(\frac{12}{6000}\right)$ : a. 3-cornered, sub-globular ; in water globular-clouded.

99. Melittis melissifolia, L. $\left(\frac{8}{6000}\right)$ : $a$. oval, opaque. $b$. somewhat drop-shaped. c. in water globular-clouded.

\section{'Verbenacez.}

100. Cyclonema myricoides, Hook. $\left(\frac{16}{6000}\right)$; spherical, echinulate ; unaltered in water.

101. Clerodendron Thomsonianum, Ball $\left(\frac{15}{6000}\right)$; oval, 3 slits; in water clouded, with a margin.

102. Clerodendron? $\left(\frac{6-12}{6000}\right): a \cdot \mathrm{som}_{\mathrm{e}-}$ what cubical, rough, with pores. $b$. and $c$. in oil. $d$. effete sack.

103. Clerodendron spicatum $\left(\frac{12}{6000}\right)$; circular, flattened, with echinate margin; in oil the echinulations scarcely visible, furrowed.

104. Lantana Radula, Sw.; oblong, a slit visible at the top; in oil the sides slit and open; in water spherical-clouded.

105. Spielmannia africana, Wild. $\left(\frac{8}{6000}\right): a$. sub-globular, 3 slits. $b$. in oil. $c$. in water.

106. Aloysia citriodora $\left(\frac{5}{6000}\right): a$. elliptic, with 3 furrows. $b$. in oil the furrows bifid. ' $c$. in oil. $d$. in water.

107. Petraa erecta, Lod. $\left(\frac{12-15}{6000}\right)$; oblong, flat, a pore at the ends; in water 3 -cornered. 


\section{ACANTHACE玉.}

108. Cyrtanthera chrysantha $\left(\frac{18-25}{6000}\right)$ : $a$. oval, $3-6$ bands. $b$. in oil the bands showing spots in one or two rows; the bands, or spots, composed of round clots, sometimes opaque, some transparent. $c$. with the flovilla bursting; in water, thick clouded.

109. Dadalacanthus macrophyllum $\left(\frac{11}{6000}\right)$; globular reticulations; in vinegar or water oily drops extruded.

110. Thunbergia Hawteyana, Wall. $\left(\frac{16}{6000}\right): a$. a coil of 7 plies. $b$. end up. c. in water.

111. Sanchesia speciosa, $\left(\frac{16}{6000}\right): a$. a coil of 8 plies, with a row of pores on the plies, oval, or nearly globular. $b$. unfolding slightly in vinegar, each pore extending fovillæ.

112. Amphiscopia Pohliana, Nees $\left(\frac{5}{6000}\right)$; oval, with minute echination.

113. Acanthus mollis, L. $\left(\frac{15}{6000}\right)$; finely reticulated, oval.

114. Aphelandra pulchervima, Hook. $\left(\frac{14}{6000}\right)$; oval, 3 slits, rough.

115. Peristrophe speciosa, Nees $\left(\frac{20}{6000}\right)$; oblong, a coil of 8 folds, pores on the coil; in water oval.

116. Goldfussia biceps, Nees $\left(\frac{25}{6000}\right)$ : $a$. narrow ellipse, a long coil, pores in the coil. $b$, spreading in vinegar.

117. Sericographis Gheisbrechtiana, Nees $\left(\frac{25}{6000}\right)$ : a. elliptic, numerous pores. $b$. in oil the coil barely visible; in vinegar oily dops extruded.

118. Barleria flava,Jacq.: $a$. oval, the extine crimped so as to make a rough exterior corering. $b$. end up. c. in water the oily drops and fovilla escaping.

119. Eranthemum crenulatus, Wall. $\left(\frac{9}{6000}\right)$; oval, 1 band visible; but in vinegar the coil becomes visible; in water globular.

120. Lankesteria parviflora $\left(\frac{15}{6000}\right)$; oval, 10 plies of coil; in water globose reticulate.

121. Fittonia gigas $\left(\frac{6}{6000}\right)$; oval, opaque; in oil with obscure bands; and in water jets of oil extruded.

122. Asystasia coromandeliana, Nees $\left(\frac{8}{6000}\right)$; sub-oblong, with depressions on either side; in oil a 3 -fold coil; in water globular.

123. Asystasia capensis, Nees $\left(\frac{10}{6000}\right)$ : $a$. sub-spherical, 16 spiral bands. b. in water.

124. Thyrsacanthus strictum, Nees $\left(\frac{15}{6000}\right): a$. oval, with 12 bands. $b$. in oil separated. $c$. in water. 125. Justicia calytriche, Hook. $\left(\frac{12}{6000}\right)$ : $a$. and $b$. sub-oblong, 12 spiral bands. $c$. in water. $d$. a bit of the spiral band detached.

126. Libonia floribunda $\left(\frac{12}{6000}\right)$; subglobular, sub-oblong, with rows of pores; separating in oil.

127. Elytraria crenulata, Vahl: $a$. oval, with spiral bands. $b$. in oil.

\section{Bignoniced.}

128. Bignonia speciosa, Hook. $\left(\frac{12}{6000}\right)$ : $a$. opaque, rough, sub-globular. $b$. in vinegar oily drops extruded.

129. Bignonia stans, L. $\left(\frac{10}{6000}\right)$; oval, with 3 furrows; in water globular. 
130. Amphicome Emodi, Royl $\left(\frac{15}{6000}\right)$; spherical, with many bands.

\section{Gesneracex.}

131. Aschynanthus speciosa $\left(\frac{8}{6000}\right)$ : $a$ smooth, oval, 3 furrows. $b$. end up. $c$. in water.

132. Gesnera insignis $\left(\frac{4}{6000}\right): a$. elliptic, 3 furrows. $\quad b$. end up. $c$. in vinegar. $d$. in water.

133. Achemenes, sp. $\left(\frac{6}{6000}\right): a$. rough, elliptic. b. empty. $c$. in water.

\section{LENTIBUTaARIA.}

134. Utriculariamontana,Jac. $\left(\frac{6}{6000}\right)$; very various in form, from oval to 3-cornered or circular; sometimes flat, circular, with 6 openings; sometimes with a grumous inside.

\section{OROBANCHEAE.}

135. Orobanche rubra, Lin. $\left(\frac{4}{6000}\right)$; minute, broad, oval, 3 slits ; in water globular, the slits just visible.

\section{SCROPHULARIEA.}

136. Calceolaria scabiosifolia, Lin. $\left(\frac{4}{6000}\right): a$. minute, simple, rough. $b$. in oil 4 valves visible. $c$. in vinegar globular, the valves turned up at the edges.

137. Schizanthus pinnatus, $R$. $P$. $\left(\frac{5}{6000}\right): a$. narrow elliptic with 3 furrows. $b$. endwise. $c$. in oil. $d$. in vinegar, with oily drops diffused. $e$. in water globular clouded.

138. Mimulus moschatus, Don. $\left(\frac{6}{6000}\right)$ : $a$. oval, rough, 3 furrows. $b$. in water spherical, clouded. I have not seen the coiled form in this species.

139. Euphrasia officinalis, $L \cdot\left(\frac{8}{6000}\right)$ : $a$. broad elliptic, rough, with deep furrow. $b$. in water.

140. Antirrhinum majus, L. $\left(\frac{7}{6000}\right)$ : a. elliptic with 3 rough bands and furrows. $b$. end up. $c$. in water.

141. Teedia lucida, Rud. $\left(\frac{6}{6000}\right): a$. oval, 6 furrows. $b$. in oil 3 bands.

142. Scrophularia nodosa, $L .\left(\frac{7}{6000}\right)$; oval, with 3 furrows.

143. Melampyrum pratense, $L \cdot\left(\frac{4}{6000}\right)$ : $a$. opaque oval. $b$. in oil with notches in the sides, 3 furrows. $c$. in water clouded globular.

144. Bartsia odontitis, Huds. $\left(\frac{8}{6000}\right)$ : $a$. oval, rough, 3 furrows. $b .3$ slits end up. c. in water globular.

145. Rhinanthus crista galli $\left(\frac{6}{6000}\right)$ : $a$. elliptic with 6 bands. $b$. end up in oil. $c$. in oil. $d$. in water.

\section{Solanaceze.}

146. Browallia elata $\left(\frac{5-6}{6000}, \frac{6-8}{6000}, \frac{8}{6000}\right.$, $\left.\frac{10}{6000}, \frac{12}{6000}, \frac{14}{6000}\right)$; multiform. 1-10. simple, 3 bands and furrows. 11, 12. circular with 4 slits. $13,14,15,16$. globular with 3 pores and bands. 17 . similar, but much larger. 18-19. a flat circle with 5 slits. 20 . with numerous irregular slits, pentagonal in band's. 21. polyhedric. 23. 3-cornered with pores. 24 . withered grain with 5 furrows. 26. with 3 furrows, the band with transverse furrows. 27. the same in oil. 
147. Nolana paradoxa, Lind. $\left(\frac{15}{6000}\right)$; sub-spherical to sub-triangular, 3 pores at each corner.

148. Solanum dulcamara, $L .\left(\frac{10}{6000}\right)$ : $a$. elliptic. $b$. in oil with 3 slits visible; in water globular.

149. Iycium americanum $\left(\frac{7}{6000}\right): a$. elliptic, 3 slits, the outer with most delicate, transverse, parallel markings; in water globular.

150. Datura sanguinea, B.\& P. $\left(\frac{10}{6000}\right)$; broad oval with 3 slits; in vinegar 3-cornered or globular.

151. Habrothamnus fusciculatus, Eud. $\left(\frac{8}{6000}\right): a$. opaque sub-oval, with 3 bands and slits. $b$. under oil the basids torn. $c$. in water globular remains of band.

152. Juanulloia parasitica, $R$. \& $P$. $\left(\frac{8}{6000}\right): a$. sub-spherical with 3 bands. b. $c$. in oil. $d$. in water.

\section{Convolvulace.s.}

153. Convolvulus Scammonia, L. $\left(\frac{22}{6000}\right)$ : a. smooth, elliptic, with 3 bands. $b$. in oil showing the slits. $c$, in water globular clouded.

154. C. arvensis, $L .\left(\frac{20}{6000}\right): a$. opaque elliptic. $b$. in oil 3 slits, with a rough outer coat with a margin. $c$. in vinegar the slits separated. 155. C. tricolor, L. $\left(\frac{9}{6000}\right)$ : a. smaller, not opaque, rough, elliptic. $b$. in end in oil.

156. Batatas pentaphyllus, Chris. $\left(\frac{24}{6000}\right)$; spherical echinate, with numerous facets, each having a pore.

157. Calystegia Sepium, Br. $\left(\frac{24}{6000}\right)$ : a. spherical, not echinate; many facets, a pore in each. $b$. in oil with an apparent margin.
158. Evovolvulus arbusculus, Poir.; opaque, sub-spherical; in oil, with a broad band.

159. Cuscuta reflexa $\left(\frac{8}{6000}\right)$; globular, opaque; in oil with an apparent margin.

160. Cuscuta Epithymum $\left(\frac{4}{6000}\right)$; oval, with 3 slits. c. in water globular.

\section{BoragnNex.}

161. Pulmonaria angustifolia, $L$. $\left(\frac{8}{6000}\right):$ a. constricted in the middle, oblong, with slight reticulations. $\quad b$. in oil, opening on each side.

162. Pulmonaria saccharata. Mill. : a. oval, with 3 pores. b. c. in oil. $d$. in water.

163. Symphytum officinale, $L .\left(\frac{6}{6000}\right)$ : $a$. in two divisions, nearly circular. $b$. in water, a halo of fovilla.

164. Myosotisscorpioides, Will. $\left(\frac{2}{6000}\right)$; minute, dumb-bell shape.

165. Mertensia maritima $\left(\frac{3}{6000}\right)$; dumb-bell shape.

166. Lycopsis arvensis, L. $\left(\frac{12}{6000}\right)$ : a. somewhat similar, but much larger. $b$. in oil the pore is the side visible, with 4 craters on the equator; not shown in the plate. $c$. in water oval.

167. Borago orientalis, L. $\left(\frac{6}{6000}\right)$ : oval, smooth. $b$. end up. $c$. in water oval.

168. Heliotropium peruvianum, $L$. $\left(\frac{5}{6000}\right): a$. oval, 3 slits. $b$. in water sub-globular.

\section{HYDROPHYLLACEX.}

169. Romanoffzsia siktiensis, Cham. $\left(\frac{4}{6000}\right): a$. elliptic. b. end up. $c$. in water, globular clouded. 
170. Nemophila atomaria, F. \& $M$. $\left(\frac{5-6}{6000}\right): a .3$-cornered, oval, with a pore at each side. $b$. in oil. $c$. end up. $d$. in vinegar cloudedglobular.

\section{Polemontacex.}

171. Gilia (Leptosyphon) androsacea, Lind. $\left(\frac{11}{6000}\right)$ : a. 3-cornered, red, opaque. $b$. in oil yellow. $c$. in water.

172. Polemonium reptans, $L .\left(\frac{8}{6000}\right)$ : $a$. globular, white. $b$. in oil the pores visible.

173. Phlox Drummondi, Hook. $\left(\frac{10}{6000}\right)$; yellow, globular, with numerous facets, with a pore in each.

174. Gilia sp. $\left(\frac{10}{6000}\right): a$. globular, with delicate parallel markings. $b$. in water with drops of oil extruded.

\section{Gentianaces.}

175. Gentiana acaulis, L. $\left(\frac{12}{6000}\right)$ : $a$. elliptic, rough. $\quad b$. in vinegar oily drops extruded.

\section{Apocyneacez.}

176. Toxicophlaa Thunbergii $\left(\frac{8}{6000}\right)$ : $a$. 6-sided. $b$. in vinegar subglobular.

177. Vinca major, L. $\left(\frac{10}{6000}\right): a$. oval, with 3 bands. $b$. in oil. c. in water.

178. Allamanda cathartica, $L .\left(\frac{20}{6000}\right)$ : $a$. hyaline, oval, with 3 bands. $b$. in water the slits still visible.

179. Arduina bispinosa, L. $\left(\frac{7}{6000}\right)$ : $a$. oval, with 3 slits. $b$. in oil. c. in water.

\section{JASMINEACEX.}

180. Jasminum nuliflorum $\left(\frac{10}{6000}\right)$ : a. oval, rough, 3 broad bands. b. in oil.

181. Fraxinus excelsior, $L$. $\left(\frac{8}{6000}\right)$ : a. rough, oval, 3 bands. $b$. in vinegar. c. in water.

\section{Mrrsinaceze.}

182. Jacquinia aurantiaca, Ait $\left(\frac{5}{6000}\right)$ : $a$. oval, rough. $\quad b$. in oil. $c$. in water globular.

183. Masa indica, Ait $\left(\frac{4}{6000}\right): a$. oval, 3 bands.

184. Clavija latifolia $\left(\frac{4}{6000}\right): a$. oval. b. in water.

\section{Primulacex.}

185. Lysimachia thyrsiformis, $L$. $\left(\frac{9}{6000}\right): a$. oval, rough. $b$. in oil. $c$. in water globular.

186. Cyclamen persicum, Mill. $\left(\frac{3}{6000}\right)$ : $a$. oval. $b$. in oil. $c$. in water.

187. Primula sinensis, Lind. $\left(\frac{3-8}{6000}\right)$ : $a$. oval. $b$. end up. $c$. in water. $\left(\frac{8}{6000}\right)$, thrum-eyed; $\left(\frac{3}{6000}\right)$, pineyed.

188. Primula auricula $\left(\frac{6}{6000}\right)$; pineyed; a band with 3 furrows.

189. Primula cortusioides $\left(\frac{3}{6000}\right)$; oval, end up, 3-cornered.

190. Primula vulgaris, Hud. $\left(\frac{5-8}{6000}\right)$; thrum-eyed, 3 furrows, each furrow bifid; $\left(\frac{4-5}{6000}\right)$; pin-eyed.

191. Primula nivalis, Pall. $\left(\frac{4}{6000}\right)$; elliptic.

192. Androsace Chamajasme, Wild. $\left(\frac{4}{6000}\right)$

193. Dodecatheon Meadii, L. $\left(\frac{2-4}{6000}\right)$; oval, end up, 3-cornered. 


\section{Plumbaginex.}

194. Plumbago cerulea, H. B. \& $K$. $\left(\frac{15}{6000}\right): a$. oval, 3 slits; bands punctulate. $b$. in oil. $c$. in water, the 3 divisions distinct.

195. Plumbago rosea, L. $\left(\frac{12}{6000}\right)$ : $a$. extine puckered, so as to look rough. $b$. in oil, 3 bands visible. c. 8 slits, opening longitudinally.

196. Armeria vulgaris, L. $\left(\frac{12}{6000}\right)$ : a. greenish, with reticulate markings. $b$. in oil, the reticulation puckered. $c$. in water, 3 bands distinct. See Lindl. Introduction, No. 22.

197. Statice Halfordii $\left(\frac{12}{6000}\right): a$. spherical, punctured surface. $b$. in oil 3 valves visible.

\section{EPACRIDEe.}

198. Leucopogon, sp. $\left(\frac{6}{6000}\right) a .0 v a l$, opaque. $b$. in oil 3 furrows visible.

199. Styphelea tubiflora, Lin. $\left(\frac{12}{6000}\right)$; hyaline, globular, with numerous echinations.

200. Epacris, sp. $\left(\frac{10}{6000}\right): a .4$ globes, 3 only visible; but $(b)$ the 4 th becoming visible in oil.

\section{Ericacex.}

201. Rhododendrum arboreum, Lin. $\left(\frac{12}{6000}\right)$; similar to the Epacris; only 3 visible at a time.

202. Arbutus Unedo $\left(\frac{10}{6000}\right)$ : a. subcubical. $b$. in oil rounded, 4 . c. effete.

203. Arctostaphylus Uva-ursi, Spr. hyaline, parallelopiped.

\section{VACCINACEE.}

204. Macleania speciosa $\left(\frac{12}{6000}\right): a$. 4-lobed, sub-pyramidal. $b$. in oil. $c$. in water.

205. Thibaudia pulcherrima $\left(\frac{8}{6000}\right)$; 8-sided.

\section{Oampanulaces.}

206. Campanula Medium, L. $\left(\frac{10}{6000}\right)$; globular, echinate.

207. Campanula hederacea, $L \cdot\left(\frac{4}{6000}\right)$ : $a$. smooth, dodecahedric. $b$. in oil.

208. Canarina campanulata, Lam. $\left(\frac{8}{6000}\right)$; sub-globular, with 3 scars.

\section{Composite.}

209. Bardenesia rosea, Lind. $\left(\frac{10}{6000}\right)$; oval, rough, polyhedric, in water globular.

210. Carduus arvensis, $L .\left(\frac{12}{6000}\right): a$. globular, echinate. $b$. in water.

211. Tragopogon pratense, $L_{\text {. }}$; dodecahedric, a pore in each facet; with numerous fine echinations on the edge of each facet.

212. Cacalia splendens $\left(\frac{12}{6000}\right) ;$ globular, echinate, with 3 furrows.

213. Centaurea scabiosa, L. $\left(\frac{12}{6000}\right)$; oval, with 3 furrows, smooth.

214. Centaurea nigra, L. $\left(\frac{10-12}{6000}\right)$; oval, with 3 slits, echinulate.

215. Centaurea Calcitrapu, L. $\left(\frac{8-12}{6000}\right)$ : with 3 forms. a. b. globular, echinulate, with 3 slits. $c$. oval, nearly smooth, 3 slits. $d$. smaller, with end up. (e) $f$. echinulate, with 3 slits, oval. $g$. in water.

216. Achillea Millefolium, $L .\left(\frac{4}{6000}\right)$; oval, with 3 furrows. 
217. Rhodanthe Manglesii $\left(\frac{8}{6000}\right)$ : $a$. globular, echinate. $b$. in oil the echination disappears, with 3 slits. c. in water with oily drops extruded.

218. Ainslea pteropoda, DC. $\left(\frac{10}{6000}\right)$ : $a$. oval, smooth. $b$. in oil bursting with 3 slits. $c$. in water.

\section{DIPSICACEA.}

219. Knautia arvensis, Coult. $\left(\frac{20}{6000}\right)$ : a. rough, globular. $b$. in oil.

\section{VALERIANACEA.}

220. Centranthus ruber, DC. $\left(\frac{12}{6000}\right)$ : $a$. echinate; it loses its echination in oil. b. 3 slits. $c$. in water subtrigonous.

\section{RUBIACEx.}

221. Higginsia macrophylla $\left(\frac{8}{6000}\right)$ : $a$. elliptic, with 3 slits. $b$. in water.

222. Burchellia capensis $\left(\frac{8}{6000}\right)$; elliptic, rough, with 3 pores.

223. Coccocypselum repens, Sw. $\left(\frac{3}{6000}\right)$; opaque oval ; in oil with furrows.

224. Galium palustre, $L .\left(\frac{7}{6000}\right): a$. elliptic, with 3 bands. $b$. in oil. $c$. in water.

225. Ixora coccinea, $L .\left(\frac{4}{6000}\right)$; oval, with 3 slits.

226. Musscenda frondosa, L. $\left(\frac{4}{6000}\right)$ : $a$. elliptic. $b$, end up.

227. Morinda umbellata, L. $\left(\frac{4}{6000}\right)$ : $a$. elliptic, rough. $b$. in oil. $c$. in water.

228. Rondeletia speciosa, Ludd. $\left(\frac{3}{6000}\right)$ : a. oral, with 3 bands. b. c. $d$. in oil. $e$. in water. $f$. in water.
229. R. fragrans $\left(\frac{2}{6000}\right)$ : a. 3-lobed. $b$. opening out in water.

230. Pentas carnea, Bent. $\left(\frac{4}{6000}\right)$; subspherical, with 3 bands; with minute crystals in the anther cells.

231. Randia longissima $\left(\frac{12}{6000}\right) ; 4$ globular lobes, as in the Ericea.

232. Palicouria, sp. $\left(\frac{20}{6000}\right)$; globular, rough; with long raphids in the anther cells.

233. Rhodospermum Gardenii $\left(\frac{16}{6000}\right)$; sub-spherical raphids in the anther cells.

234. Cephaelis spicata $\left(\frac{10}{6000}\right)$; opaque, globular, with 3 slits faintly visible; with minute raphids in the anther cells.

235. Manettia micans $\left(\frac{12}{6000}\right)$ : a. opaque, oval. b. end up. $c$. in oil, rough furrows, with numerous raphids in the anther cell.

\section{Caprifolitacex.}

236. Sambucus nigra, $L .\left(\frac{10}{6000}\right): a$. elliptic, with 3 furrows and bands. $b$, in oil 2 slits visible at once. $c$. in water.

237. Viburnum Tinus, $L .\left(\frac{9}{6000}\right)$ : $a$. oval, rough, 3 furrows. $\quad b$. in oil. $c$. in water.

238. Lonicera Periclymenum, L. $\left(\frac{10}{6000}\right)$; spherical, echinate ; in oil.

239. Linnaea borealis, L. $\left(\frac{10}{6000}\right): a$. sub-spherical, echinate, with 3 slits. $b$. in water with 3 pores.

240. Diervillia (Wagetia) rosea, Lind. $(\overline{6000})$; sub-spherical, rough, with 3 slits.

241. Abelia triflura, $B r \cdot\left(\frac{12-15}{6000}\right): a$. oval, crystalline. $\quad b$. in oil, the 
outer coat becoming visible. $c$. in oil, end up. $d$. in vinegar or water, swollen into a sphere with 3 bands and 3 large craters, with fovillæ bursting out of them.

\section{CoRTaCGe.}

242. Cornus paniculata, L. Han. $\left(\frac{5-6}{6000}\right)$; oval, with a broad band.

243. Aucuba japonica, Thun. $\left(\frac{12}{2000}\right.$, $\left.\frac{12}{6000}\right)$ : $a$. oval, rough, 3 slits. $b$. in oil, trigonous, with drops of oil extruded.

\section{UMBELLIFERE.}

244. Caucalis anthriscus $\left(\frac{6}{6000}\right)$; constricted in the middle, oval, with a band and a crossband with pores.

245. Pastinaca sativa, $L$. $\left(\frac{7}{6000}\right)$; oval, slightly furrowed, with 3 pores.

246. Hacquetia epipectis, DC. $\left(\frac{10}{6000}\right)$ : $a$. elliptic, with 3 furrows. $b$. in oil. co in water.

\section{Ficordex.}

247. Mesembryanthemum spectabile, Ham. $\left(\frac{6}{6000}\right): a$. spherical, echinate, with 3 pores. $b$. in oil the echinations disappear.

\section{Cacteas.}

248. Rhypsalis salicornicedes, Ham. $\left(\frac{8}{6000}\right): a$. globular, 1 pore visible. $b$. in water no pore visible.

249. Phyllocactus multiflorus $\left(\frac{8}{6000}\right)$ : a. sub-spherical, echinate, with 3 bands and furrows. $b$. in cil showing the ends of the furrows. $c$. in water the echinations disappear, and drops of oil are extruded.
250.Mammillariaquinquecaulis $\left(\frac{10}{6000}\right)$; broad, oval, with broad band.

251. Cereus Phyllanthus, DC. $\left(\frac{15}{6000}\right)$ : $a$. broad oval, echinate. $b$. with a rough ring. $c$. in vinegar, end up, 3 furrows, or with each furrow dividing into 6 slits.

252. Opuntia monacantha, Ham. $\left(\frac{25}{6000}\right)$; varies from globular to cubical, with 6 or 8 large pores and bands.

\section{BegoniaCEe.}

253. Begonia, $s p .\left(\frac{4}{6000}\right): a$. minute, narrow, elliptic. $b$. in vinegar, oval, with 2 pores visible.

\section{LOASUCEA.}

254. Loasa, $s p \cdot\left(\frac{6}{6000}\right)$; elliptic, with 3 furrows.

\section{Cocurbitaces.}

255. Trichosanthes Anguina, L.: a. oval, with pores on the equator. $b$. in water, with 3 pores protruding.

256. Begonia dioica, L. $\left(\frac{10}{6000}\right)$; elliptic, rough, with furrows. $a$. in oil. $b . c$. in water, globular, with a margin.

257. Momordica Charantia, L. $\left(\frac{12}{6000}\right)$ : $a$. opaque, spherical, dark yellow. $b$. in oil the yellow oil is dispersed in the olive oil.

\section{Onagraces.}

258. Trapa bispinosa, Roxb. $\left(\frac{8}{6000}\right)$; sub-spherical, 3 slits, no raphids in the anthers. Therefore I think it belongs rather to the Halagorea, as placed by Lindley, than to the Onagracea, which 
have numerous raphids in every other species of the family, and the pollen is quite different in this from all the rest of this family.

259. Circaa Lututiana, $L$. $\left(\frac{10}{6000}\right)$; triangular, a pore at each end, numerous raphids in the anther cell.

\section{LYTHRACEA.}

260. Lythrum Salicaria, L. $\left(a \cdot \frac{4}{6000}\right.$, d. $\left.\frac{8-10}{6000}, g \cdot \frac{12-15}{6000}\right): a$. pores, a yellow anther of a short staminal form, sub-globular, a pore protruding. $b$. in oil. $c$. in water. d. pores, a hairy yellow anther of a long stamened form, with 3 bands and slits. $f$. the end up. g. pores a blueanther from long stamen, 3 slits. $j$. in vinegar, 3-cornered.

261. Cuphia, sp. $\left(\frac{10}{6000}\right)$ : a. triangular, rough. $b$. in oil opening by 3 slits. $c$. end view.

262. Nesaa salicifolia $\left(\frac{6}{6000}\right): a$. broad, oval. b. globular in water.

\section{Melastomacez.}

263. Centranthus inequalis $\left(\frac{4}{6000}\right)$; elliptic, with 3 slits.

264. Medinilla magnifica, Lind. $\left(\frac{2}{6000}\right)$; similar, very small.

265. Monachata multiflora $\left(\frac{6}{6000}\right)$ : a. broad, oval, rough, 3-6 bands. $b$. in water.

\section{Mrrtacex.}

266. Darwinia (Gentyllis) fuschioides $\left(\frac{8}{6000}\right)$; triangular, a pore at each corner, as in Protancea. The anther-cells are very beautiful, with numerous crown-shaped cells.

\section{Haloragex.}

267. Gunnera scabra, R. \& $G$. $\left(\frac{7-8}{6000}\right): a$. sub-spherical, with 3 lobes. $b$. in oil showing the lobes more distinctly. $c$. in vinegar, with the 3 lobes and fovillæ coming out of the slits.

\section{HaMaMelide无.}

268. Hamamelis virginica, Lam. $\left(\frac{12}{6000}\right): a$. elliptic. $b$. in oil. $c$. globular in water.

\section{DROSERACE丑.}

269. Drosera rotundifolin, $L .\left(\frac{7-8}{6000}\right)$; sub-globular, echinulate, with 3 slits and bands.

270. Drosera anglica, Hud. $\left(\frac{9-12}{6000}\right)$; larger, with 3 distinct lobes, echinulate.

\section{Crassulaces.}

271. Grammanthes chloriflorus, $D C$. $\left(\frac{5}{6000}\right): a$. elliptic, with 3 slits and bands. $b$, in vinegar 3-cornered.

272. Cotyledon umbilicus $\left(\frac{6}{6000}\right): a$. elliptic. $b$. end up to show the ends of the furrows.

\section{Saxifragacex.}

273. Philadelphus coronaria, $L$. $\left(\frac{6}{6000}\right)$ : $a$. elliptic, with 3 furrows. $b$. in water globular.

274. Hydrangea hortensis, Sims. $\left(\frac{4}{6000}\right)$; similar, but with numerous raphids in the anther, which are also found in other species of the same genus, and in Decumaria.

275. Parnassia palustris, L. $\left(\frac{7}{6000}\right)$; elliptic, rough, with 3 slits and bands. 
276. Saxifruga aizoides, L. $\left(\frac{6}{6000}\right)$ : $a$. elliptic, with delicate markings on the bands, and 3 slits. $b$. in oil. $c$. in water.

277. Astilbe (Hoteia) japonica $\left(\frac{4}{6000}\right): a$. oval, roughish. $b$. in oil. $c$. in vinegar.

278. Ribes sanguineum, Purs. $\left(\frac{9}{6000}\right)$ : a. globular, with many pores. $b$. in vinegar pores protruded.

279. Ribes rubrum, L. $\left(\frac{8}{6000}\right)$; globular, with a broad band and several pores.

280. Ribes aureum, Pur's. $\left(\frac{8}{6000}\right)$ : a. prismatic, with pores on each side. $b$. globular in water.

281. Ribes grossularia, L. $\left(\frac{5-10}{6000}\right)$ : a. two forms; one globular, as in 279 . $b$. the others oval, with 3 bands. $c$. in water.

\section{ROSACE正.}

282. Fragaria vesca, $L .\left(\frac{10}{6000}\right)$; elliptic, 3 slits and bands, delicately marked.

283. Pyrus domestica, $\dot{L} \cdot\left(\frac{6}{6000}\right)$; simple, elliptic, with 3 slits.

284. Nuttallia cerasiformis, Herb. $\left(\frac{8}{6000}\right)$; the bands delicately marked.

285. Spiraa palmata, Pall. $\left(\frac{3-4}{6000}\right)$; globular, with 3 slits and bands. a. b. c. in oil. $d$. in vinegar.

286. Sanguisorba officinalis, $L$. $\left(\frac{10}{6000}\right): \quad a$. oval, with 3 slits. $b$. in oil. $c$. in vinegar 6 slits visible.

287. Poterium Sanguisorba, $\left(\frac{8}{6000}\right)$; globular, with 3 bands and 3 pores.

\section{LEGUMTNOSI.}

288. Ulex europan, $L .\left(\frac{10}{6000}\right)$; elliptic, 3 slits and bands, which are of deep yellow, and rough.

289. Trifolium subterraneum, $L$. $\left(\frac{6}{6000}\right)$; oval, with 3 slits, rough, with delicate markings.

290. Trifolium medium, L. $\left(\frac{6}{6000}\right)$; rough, with 3 slits and bands.

291. Lathyrus. Aphaca, L. $\left(\frac{10}{6000}\right)$; oblong, rough, with broad bands.

292. Vicia sativa, $L .\left(\frac{9}{6000}\right): a$. oblong, rough, with delicate curved markings on the bands. $b$. end up. $c$. in water with curved markings.

293. Saraca (Jonesia) Asoka, Roxb. $\left(\frac{6}{6000}\right): a$. oval, with 3 bands. $b$. the 3 bands end up.

294. Cussia biflora, L. $\left(\frac{15}{6000}\right): a$. long, elliptic, with 3 bands. $b$. end up.

295. Cercis Siliquastrum, L. $\left(\frac{5}{6000}\right)$; oval, with 3 rough bands, with delicate markings.

296. Arachis hypogaa, L. $\left(\frac{10}{6000}\right)$ : $a$. oval, with slight reticulation in oil. $b$. in water 3 rough bands still visible.

\section{ANACARDIACETE.}

297. Rhus Cotinus, L. $\left(\frac{6}{6000}\right)$; oval, with 3 rough bands.

\section{Sapindacex.}

298. Acer pseudoplatanus, $L \cdot\left(\frac{12}{6000}\right)$ : oval, with 3 bands; rough, with delicate markings, and 3 slits.

299. Pavia rubra, L. $\left(\frac{8}{6000}\right)$; oval, with broad bands.

300. Xanthoceras sorbifolia $\left(\frac{10}{6000}\right)$ : a. oval, with minute echinations, 
3 bands and slits. $b$. end up. $c$. in oil the echinations disappearing. $d$. in water.

\section{Rhamacez.}

301. Pomaderris elliptica, Labil. $\left(\frac{6-10}{6000}\right)$; polyhedric, with a pore on each facet.

302. Ceanothus Lobbianus $\left(\frac{7}{6000}\right)$; oval, with 3 slits and bands.

\section{Ceitastrineze.}

303. Euonymus europæens, L. $\left(\frac{10}{6000}\right)$; globular, rough, with 3 slits.

\section{ILICINEAE.}

304. Ilex aquifolium, $L \cdot\left(\frac{7}{6000}\right): a$. oval, with 3 rough bands, the echinations blunt. $b$. in oil. c. in vinegar.

\section{Otacines.}

305. Olax Wightiana $\left(\frac{5-6}{6000}\right)$; flat, 3-cornered.

306. Liriostoma villosissima $\left(\frac{8}{6000}\right)$; pyramidal, 3-cornered, like the Myrtacea.

307. Xeminia americana, L. $\left(\frac{4}{6000}\right)$; oval, with 3 oval craters.

308. Strombosia javanica $\left(\frac{4}{6000}\right)$; oval, with but 1 slit found,

309. Chenophora parviflora $\left(\frac{10}{6000}\right)$; disciform, with 6 pores on the equator.

310. C. crassifolia $\left(\frac{11}{6000}\right)$; disciform: $a$. profile, 3 pores on the band. $b$. full view, with 3 pores. $c$. in water.

311. Aptandra Benthami $\left(\frac{9}{6000}\right)$ : a. oval. $b$. in oil, polygonous.
Meliacede.

312. Turraa $\left(\frac{12}{6000}\right) ;$ 3-cornered, with 3 pores.

\section{OchNaCez.}

313. Gomphia decumbens $\left(\frac{5}{6000}\right)$; yellow, elliptic.

\section{Stmardbice}

314. Brucea antidysenterica $\left(\frac{8}{6000}\right)$ : a. rough, elliptic, with 3 slits. b. in water, globular.

\section{RUTACEA.}

315. Adenandra fragrans $\left(\frac{8-10}{6000}\right)$. Two forms, one larger: $a$. a narrow oblong, somewhat central upwards, with apparently 2 pores. $b$. but in oil one can find 3 to 6 longitudinal rough bands, with 1 or 2 cross-bars. c. larger form. d. small form ; 6 interrupted bands. e. 2 cross-bars. $f$. 6 bands. $g$. small form. $h$. transparent small form. $k$. in vinegar. l. the longitudinal band assuming the form of a ring.

316. Galepia Ruldenii $\left(\frac{9}{6000}\right)$; globular, rough, with 4 large craters on the equator.

317. Diosma errcoides, Thom. $\left(\frac{11}{6000}\right)$ : a. elliptic, with 3 bands and slits. $b$. in water, with drops of oil protruding.

318. Skimmia oblata $\left(\frac{11}{6000}\right)$; rough, elliptic.

319. S. japonica $\left(\frac{10-11}{6000}\right)$ : a. oblong with 8 bands. $b$. end up. 
320. Eriostemon castratum $\left(\frac{12}{6000}\right)$; oblong, with glandular dots.

321. Boronia megastigma $\left(\frac{8}{6000}\right)$; globular, with 3 pores.

322. Correa speciosa $\left(\frac{10}{6000}\right): a$. elliptic. $b$. with 3 slits and pores. $c$. end up.

\section{GERANIACEE. \\ Gerantex.}

323. Geranium Robertianum, $L$. $\left(\frac{15}{6000}\right): a$. globular, echinate. $b$. in oil, echination becoming blank. $c$. in water.

324. Erodium moschatum, L. $\left(\frac{18}{6000}\right)$ : a. opaque, globular, rough. $b$. in oil, finely marked. $c$. in water, with 3 bands.

\section{Pelargonla.}

325. Pelargonium unifolium $\left(\frac{16}{6000}\right)$ : $a$. broad, oval, rough, almost echinate. $b$. end up, showing the 3 slits. $c$. in oil, sub-globular.

326. Tropceolum majus, $L$. $\left(\frac{10}{6000}\right)$ : $a$. broad, oval, with 3 rough bands and slits. $b$. 3-cornered, end up.

327. T. tricolor $\left(\frac{6-8}{6000}\right)$ : a. narrow, oblong, curved. $\quad b$. in oil, showing delicate markings.

\section{Viviane.e.}

328. Viviana grandiflora $\left(\frac{8}{6000}\right)$; from the Kew herbarium. Globular, oprque, but in oil rough, varicose.

\section{LIMNANTHE.}

329. Limnanthes alba $\left(\frac{5}{6000}\right)$; crescentshaped, with 4 or 5 cells.
330. L. pulchella $\left(\frac{5-6}{6000}\right)$; smaller, with 2 or 3 cells.

\section{BALSAMINEE.}

331. Impatiens Royliana $\left(\frac{4}{6000}\right)$; hyaline, oblong, with numerous raphids in anther.

332. Impatiens $s p \cdot\left(\frac{8}{6000}\right): a$. broad, oval, with rough bands. $b$. in water, with 3 bands visible; minute raphids in anther.

333. Impatiens racemosa, Edg. $\left(\frac{7-10}{6000}\right)$ : $a$.rough, oval. b.inoil. $c$.in water.

\section{Oxamidex.}

334. Oxalis Autosella, L. $\left(\frac{6}{6000}\right)$; hyaline, globular, shining.

335. O. americana, L. $\left(\frac{8}{6000}\right)$; subspherical, with 3 bands.

336. O. Marlii $\left(\frac{5}{6000}\right)$; yellow, elliptic, with 3 slits.

337. O. carnosa, Cam. $\left(\frac{10}{6000}\right)$; globular, rough, with 3 slits.

\section{MaLPIGHIACER.}

338. Malpighia sp. $\left(\frac{12}{6000}\right)$; globular, with rough projections.

339. M.punicea $\left(\frac{12}{6000}\right)$; opaque, globular, with faint slits barely visible.

\section{LINACEX.}

340. Linum grandiflorum, Dep. $\left(\frac{12}{6000}\right)$; globular, fine network, with 3 pores.

\section{Sterculiacex.}

341. Thomasia purpurea $\left(\frac{8}{6000}\right): a$. oval, with a spot on either side. $b$. in oil, with a cross-bar. $c$. in 
water, globular, with crossband.

342. Thomasia solanacea $\left(\frac{4}{6000}\right)$; oval, with 3 slits and bands.

-343. Hermannia angularis, Jaef. $\left(\frac{6}{6000}\right)$; polyhedric.

344. Hermannia plicata $\left(\frac{6}{6000}\right): a$. globular, with a rough band.

345. Hermannia althrooides $\left(\frac{5}{6000}\right)$ : a. globular, with several pores. $b$. in oil. $c$. in water,3-cornered.

346. Mahernia vestita $\left(\frac{2-6}{6000}\right)$; aval, - minute.

347. Rulingia corymbosa $\left(\frac{5-6}{6000}\right)$; globular, with broad rough band.

348. Dombeya sp. $\left(\frac{12-13}{6000}\right)$; globular, echinate, with 3 pores, like the Malvaceæ.

\section{DiPTEROCARPECEA.}

349. Shorea robusta, Gart. $\left(\frac{4}{6000}\right)$; (from herbarium), oval, minute.

\section{Tenstramiacex.}

350. Camellia Thra Bohea (L.) $\left(\frac{10}{6000}\right)$ : a. elliptic, with 3 slits, and numerous oil dots. b. c. d. in vinegar the oily drop diffused ; in water (e) triangular or globular.

\section{HYPERICINEX.}

351. Hypericum andrascmum, $L$. $\left(\frac{8}{6000}\right)$; elliptic, in water globular.

\section{Portulacacez.}

352. Lewisia brachycalyx $\left(\frac{15}{6000}\right)$; opaque, spherical, with 3 slits.

353. Claytonia caroliniana, Mill $\left(\frac{10-12}{6000}\right)$; globular, with 3 slits.
354. Calandrina, sp. $\left(\frac{14}{6000}\right)$; globular, with pores on the equator.

\section{CaryophyLlacede.}

355. Stellaria graminea, $L$. $\left(\frac{6}{6000}\right)$; dodecahedric, a pore in each facet.

356. Lychnis Flos cuculi, L. $\left(\frac{16}{6000}\right)$; polyhedric.

357. L. Chalcedonica, L. $\left(\frac{10-12}{6000}\right)$; icosahedric.

358. Saponaria altissima $\left(\frac{5-6}{6000}\right)$; subspherical or dodecahedric.

359. Sagina procumbens, $L$. $\left(\frac{4}{6000}\right)$; dodecahedric.

360. Spergularia rubra, L. $\left(\frac{5}{6000}\right)$; elliptic with 3 slits. I find the similar form in the Indian species-a. in S. fallax. Not in any other species of the family I have examined except Spergula.

\section{TAMARISCINEE.}

361. Tamarix gallica, L. $\left(\frac{4}{6000}\right): a$. oval, 3 slits. $b$. in oil. $c$. end up. $d$. in water.

\section{VochYSIACEx.}

(All from the dried specimen.)

362. Erisema violacea, Mart. $\left(\frac{4}{6000}\right)$; globular.

363. Qualia cordata, Spr. $\left(\frac{4}{6000}\right)$; globular with 3 broad bands.

364. Trigonialavis, $A u b .\left(\frac{6}{6000}\right)$; oval, with 3 slits.

365. Callisthene minor, Mart. $\left(\frac{5}{6000}\right)$; 3-cornered.

366. Voichysia Tucanorum, Mart., globular, with 3 slits. 


\section{Polygatacex.}

367. Hymenanthera dentata, R.B. $\left(\frac{8}{6000}\right)$; elliptic, with 3 broad bands and slits.

368. Polygalagrandiflor $a\left(\frac{14}{6000}\right)$; oval, with numerous prism bands, like rods.

369. P. bracteata $\left(\frac{10}{6000}\right)$ : a. oval as above. $b$. in oil, the bands apparently broken in 3 places.

370. P. vulgaris, $L \cdot\left(\frac{8}{6000}\right): a$. oval, with a constriction in themiddle. b. looking like an arch in form in oil or water.

\section{TremandRex.}

371. Tremandra ericifolia $\left(\frac{4}{6000}\right)$; hyaline, with 3 broad bands.

372. Tetratheca verticellata, Pax. $\left(\frac{8}{6000}\right): a$. oval, the end abruptly cut off. $b$. in vinegar trigonous.

\section{Pittosporaceze.}

373. Pittosporum undulatum, And. $\left(\frac{8-9}{6000}\right)$; pale yellow, elliptic, with 3 bands and slits.

\section{Buxinez.}

374. Cochleosper'mum Gossypium,DC. $\left(\frac{5}{6000}\right)$; rough, but in oil smooth, with 3 slits. (From herbarium.)

\section{VIOLARIACEXE.}

375. Viola odorata, $L .\left(\frac{12}{6000}\right): a$. oval, with 3 bands and slits. $b$. c. in vinegar, bands broader and ultimately bursting, and becoming globular with oil, drops protruding. $d$. in water oval or globular.

376. Viola cornuta, L. $\left(\frac{12-20}{6000}\right)$ : a. b. broad, oval, with 2 cross-bands. $c$. in oil. $d$. in vinegar globu- lar, with 4 annulars, the bands with globules of oil protruding. $e$. in water globular, the crossbands still visible. There is also a form similar to No. 376 , with only the 3 bands.

377. Viola perfection, hybrid, said to be from $\left(\frac{20}{6000}\right)$ : $a$. bred by Clibran of Altrincham, cornuta and tricolor; oblong, with 5 bands. $b$. c. in oil. d. in vinegar subglobular. e. an effete sack.

378. Viola arvensis, DC. $\left(\frac{20}{6000}\right)$ : $a$. broad, oval. $b$. in oil with 5 bands; in water or vinegar the 5 bands spreading out pentagonally.

\section{RESEDACER.}

379. Reseda odorata, L. $\left(\frac{12}{6000}\right): a$. oval, broad bands, and 3 slits. c. in water globular, with drops of oil diffused.

\section{Capparidex.}

380. Sterephoma cleomoides, Spr. $\left(\frac{6}{6000}\right)$; opaque, oval with broad bands.

\section{Crdchfer}

381. Brassica oleracea, L. $\left(\frac{8}{6000}\right)$ : a. oval, rough, with a fine band. $b$. in water ovoid, with 3 slits.

382. Cheiranthus Cheiri, L. $\left(\frac{6}{6000}\right)$ : $a$. oval, rough. $b$. in oil, with 3 slits. $c$. in water ovoid.

383. Iberis umbellatus, $L$. $\left(\frac{4}{6000}\right)$ : $a$. ovoid, rough. $b$. in oil with a margin. c. in water ovoid.

384. Sisymbrium officinale, L. $\left(\frac{6}{6000}\right)$; oval roughish, with delicate marking.

385. Schizopetalon Walkeri, Linn. $\left(\frac{6}{6000}\right): a$. oval, very rough. $b$. in 
oil. c. $d$. vinegar, 3 slits. $\quad$ e. in water, globular, with concentric circles.

\section{Papateraceze.}

386. Corydalis solida, Smith $\left(\frac{7}{6000}\right)$; globular, with a cross-band.

387. C. cava, Schizy $\left(\frac{8}{6000}\right)$; globular, rough.

388. C. corniculata, DC. $\left(\frac{9}{6000}\right)$; similar to No. 386, but smoother.

389. C. capnoides, Pun. $\left(\frac{10}{6000}\right)$; globular, rough, with minute reticulation; in oil, with 3 pores.

390. Dielytra canadensis, $D C$. $\left(\frac{8}{6000}\right): a$. oval, with fine reticulation, with racemose round spots, with 3 slits. $b$. spherical in water, with drops of oil diffused.

391. Chelidonium majus, Mill $\left(\frac{5}{6000}\right): a$. yellow, globular, with numerous round spots. $b$. 3sided at the end, with 3 slits.

392. Bocconia cordata, WS. $\left(\frac{4}{6000}\right)$; pale, spherical, spotted.

393. Papaver dubium, L. $\left(\frac{8}{6000}\right)$; elliptic, roughish, with 3 slits.

394. P. rheas, $L .\left(\frac{4}{6000}\right): a$, oval, with 3 slits. $b$. globular in water.

395. P. somniferum, $L$. $\left(\frac{10}{6000}\right)$; elliptic, pale.

396. P. bracteolatum, Lind. $\left(\frac{2}{6000}\right)$; purple, subfusiform, with 3 slits : in oil elliptic, in water globular.

397. Eschscholtzia californica, Cham. $\left(\frac{10}{6000}\right): a$. elliptic, yellow with 6 slits and furrows. $b$. seen end up.

\section{Sarraceniaceze.}

398. Sarracenia flava, L. $\left(\frac{7}{6000}\right)$; hyaline, oval, withered-looking in natural state or in oil, with 6 slits, but in water swelling into a globe, with 6 (or 7) pores on the edges.

\section{NYMPHEACEE.}

399. Nelumbium speciosum, Wild. $\left(\frac{12-16}{6000}\right): a$. oval, rough. $b$. in oil roughness is more visible, with 3 furrows. $c$. end up.

400. Nymphaa versicolor, Roxb. $\left(\frac{12}{6000}\right)$; oval, with 3 bands. (United to Lotus in Hook's Flora.)

401. N. Lotus, L. $\left(\frac{10}{6000}\right)$; oval or sub-globular, hyaline, opening with 1 slit.

402. Nuphar luteum, Smith $\left(\frac{12}{6000}\right)$; oval, echinate, 1 slit only apparent.

\section{BERBERIDE巫.}

403. Berberis japonica, Thom. $\left(\frac{10}{6000}\right)$ : $a$. spherical, with broad band.

404. B. asiatica, Roxb. $\left(\frac{10}{6000}\right)$; subspherical or dodecahedric, with a pore in each facet.

405. B. dulcis, Sweet. $\left(\frac{8}{6000}\right)$; oval, with 3 slits.

406. Bongardia Rowalfi $\left(\frac{12}{6000}\right)$; oval, rough.

407. Epimedium alpinum, L. $\left(\frac{10}{6000}\right)$ : $a$. elliptic, roughish, with delicate markings. $b$. showing the end with 3 furrows.

408. Diphylleia cymosa, Mid. $\left(\frac{10}{6000}\right)$; globular, echinate.

409. Holbellia latifolia, Wall. $\left(\frac{4-5}{6000}\right)$ : $a$. oval, with 3 slits. $b$. end up. 


\section{AnNonacese.}

410. Meliusa velutina $\left(\frac{10}{6000}\right)$; oval, with 3 bands and slits.

\section{Magnoliacex.}

411. Magnolia glauca, L. $\left(\frac{9}{6000}\right)$; fusiform, with but 1 slit, as in the Liliacere.

\section{WINTEREX.}

412. Drymis Winterii, L. $\left(\frac{8}{6000}\right)$; sub-globular, rough, with 3 slits and bands.

\section{RANUNCULACEX.}

413. Paonia corallina, Retz. $\left(\frac{20}{6000}\right)$ : $a$. elliptic, rough, 3 slits. $b$. in vinegar trigonous.

414. Aconitus Napellus, L. $\left(\frac{5}{6000}\right)$; oval, with 3 slits.

415. Helloborus lividus, Ait. $\left(\frac{7}{6000}\right)$; globular, with 3 slits.

416. Aquilegia vulgaris, $L$. $\left(\frac{6}{6000}\right)$; oval, smooth, 3 slits.

417. Adonis pyrenaica, DC. $\left(\frac{8}{6000}\right)$ : $a$. oval, with 3 bands, rough. b. end up.

418. Ranunculus bulbosus $\left(\frac{5}{6000}\right): a$. oval, smooth. $b$. in vinegar a protuberance emerging.

419. Ranunculus auricomus, $L$. $\left(\frac{4}{6000}\right): a$. oval, with a rough band. $b$. in water clouded-globular.

420. Ranunculus flammeus, $L \cdot\left(\frac{5}{6000}\right)$ : $a$. oval, rough, 3 bands. $b$. in vinegar globular, with 3 slits.

421. Ranunculus Lingua, L. $\left(\frac{9}{6000}\right)$ : $a$. oblong, in oil. b. globular, with a rough band. $c$. in vinegar the band broader. $d$. in water globular, the marks of the bands still visible.

422. Ranunculuslomatocarpus $\left(\frac{8}{6000}\right)$ : a. sub-oblong, with 2 broad rough bands. $b$. in oil.

423. Ranunculus arvensis, L. $\left(\frac{15}{6000}\right)$; spherical, echinate.

424. Anemone coronaria, L. $\left(\frac{10}{6000}\right)$; purple; icosahedric, compressed laterally.

425. Anemone pulchella $\left(\frac{9}{6000}\right)$; oval, rough, with 3 slits.

426. Anemone pavonina, Lois $\left(\frac{10}{6000}\right)$; spherical, verrucose, rounded knobs.

427. Anemone canadensis $\left(\frac{5}{6000}\right): a$. oval, with transverse markings. $b$. in oil rough, with a smooth band. $c$. end up.

428. Anemone alpina, L. $\left(\frac{6}{6000}\right)$; subspherical, rough.

429. Anemone narcissiflora, $L$. $\left(\frac{5}{6000}\right)$; oval, rough.

430. Thalictrum flavum, L. $\left(\frac{4-7}{6000}\right)$; of various sizes, sub-spherical with a broad equatorial band, bearing many pores, and a large crater at either end.

431. Thalictrum majus, L. $\left(\frac{4}{6000}\right)$; dodecahedric.

432. Thalictrum tuberculatum $\left(\frac{4}{6000}\right)$; polyhedric.

433. Thalictrum glaucum, Desp. $\left(\frac{4-6}{6000}\right)$; two forms: $a$. elliptic. b. spherical with 6 bands.

434. Clematis Jackmani $\left(\frac{6-7}{6000}\right): a$.icosahedric. $b$. in vinegar, with 6 bands. $c$. in water. 
435. Clematis orientalis, $L .\left(\frac{6}{6000}\right)$; globular, opaque.

436. Clematis Flammula, L. $\left(\begin{array}{c}4 \\ 6000\end{array}\right)$ : $a$ oval. b. end up.
437. Clematis viticella, $L \cdot\left(\frac{5}{6000}\right)$; oval. rough, with 3 slits.

438. Clematis montana, Buch. $\left(\frac{7}{6000}\right)$; oval, rough, with 3 slits.

\section{Errata.}

Page 3, line 11 from top, for Conferæ read Coniferæ.

, 3, , 14 , , Plantagine read Plantagineæ.

"3, " 2 from foot, ,, 429-432 read 430-433.

"6, " 6 " , , Caprifolaciaceæ read Caprifoliaceæ.

, 7, , 7 from top, , 425 read 423 .

, 9, " 13 " after (R.), ib., read iv. instead of vii.

, 9, " 26 , for Exogens read Endogens.

. 39, " 2 " for Lampsana read Lapsana. 

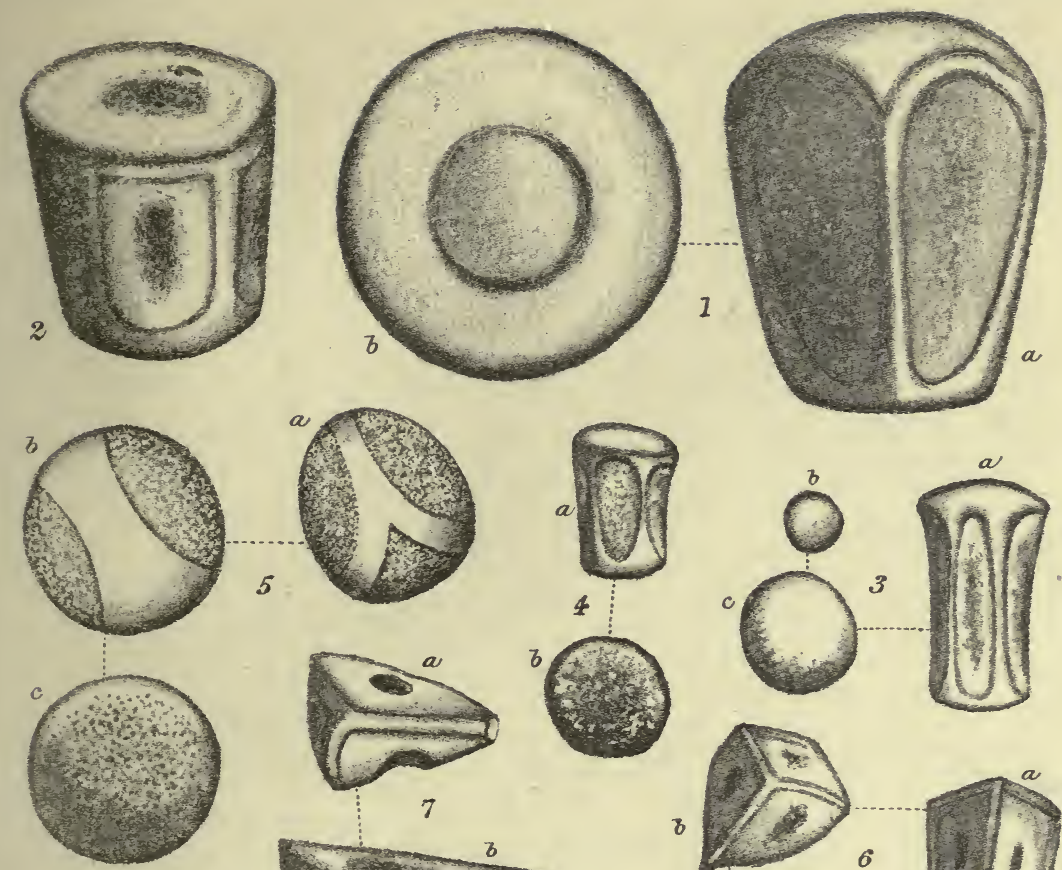

\section{7}

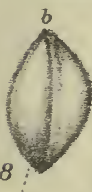

10
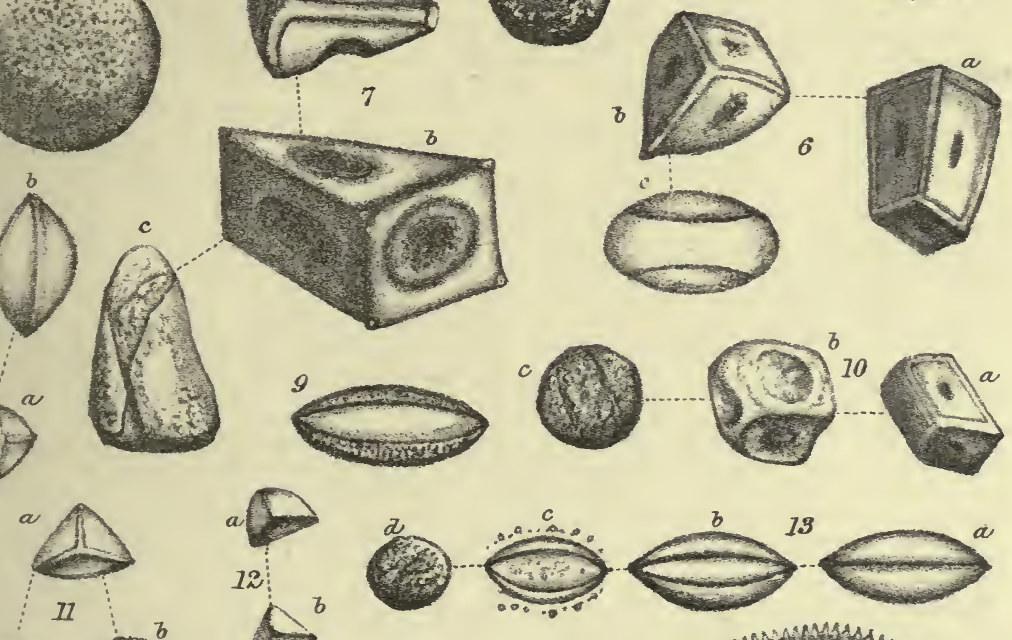

(in)
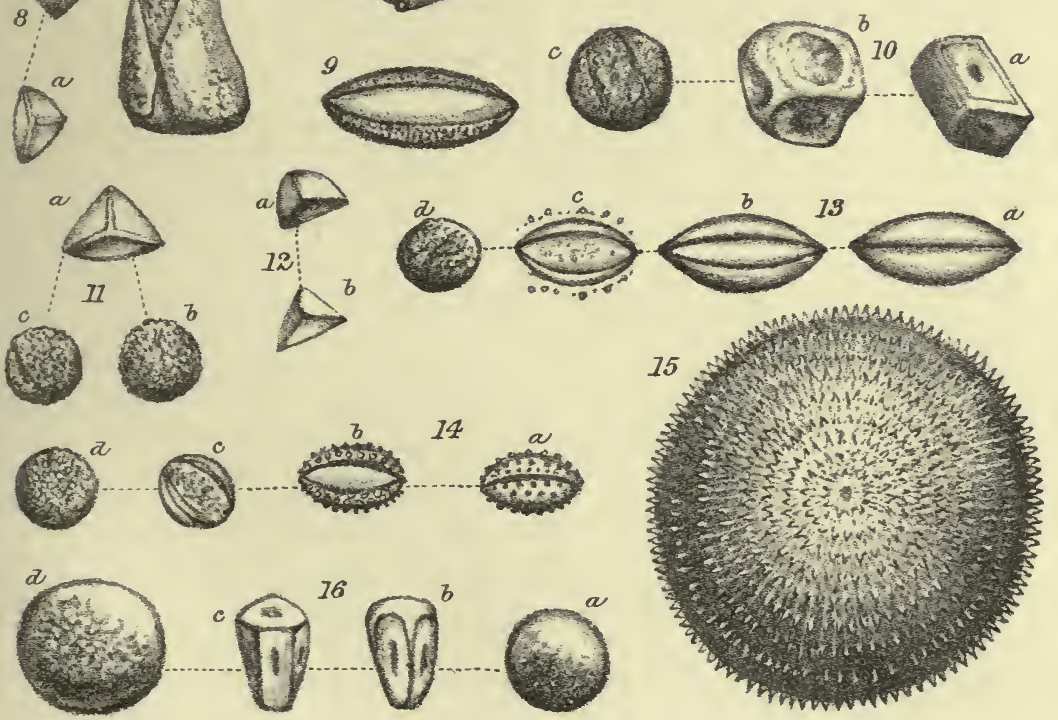

C. Berjeau, Iith, 


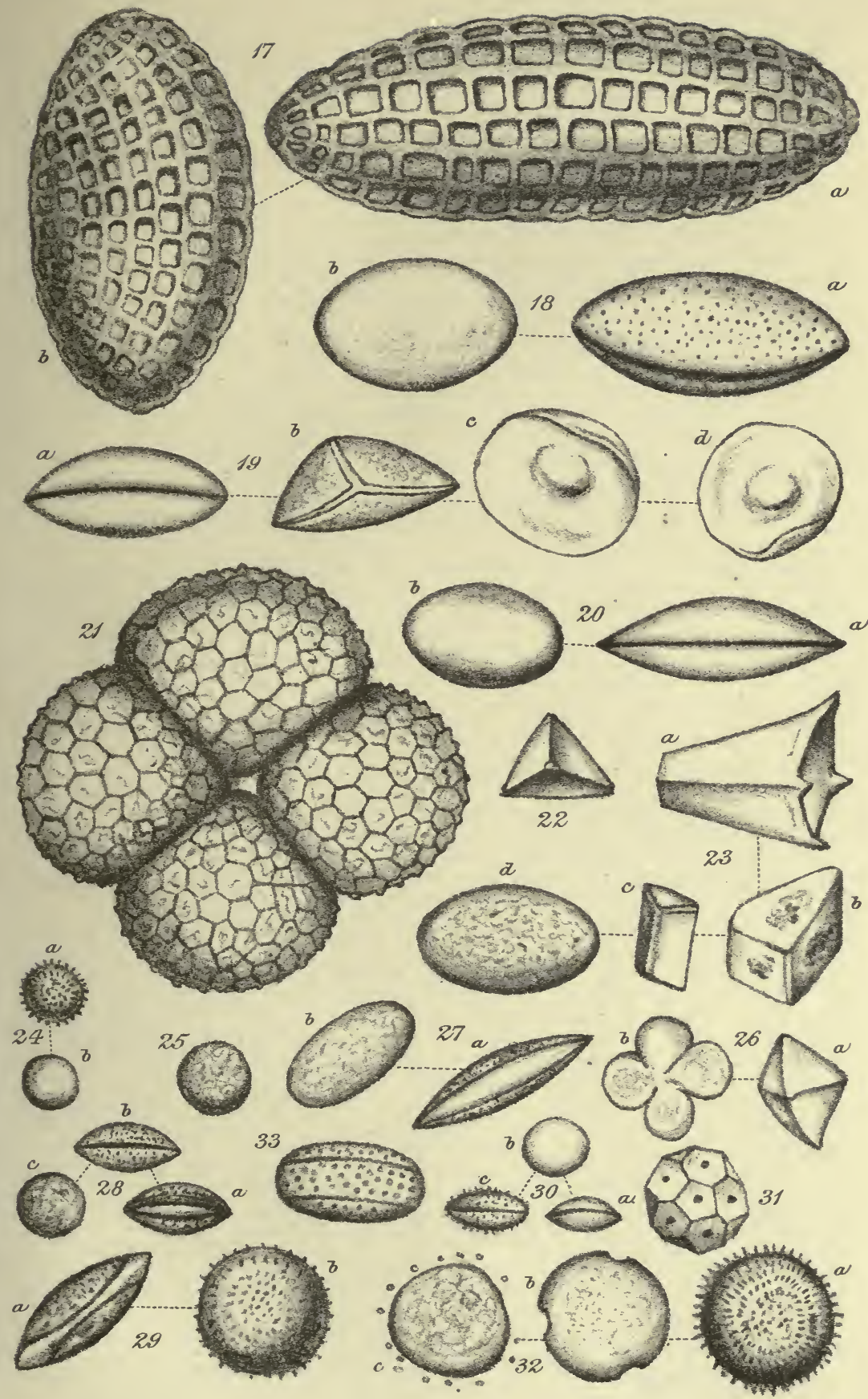


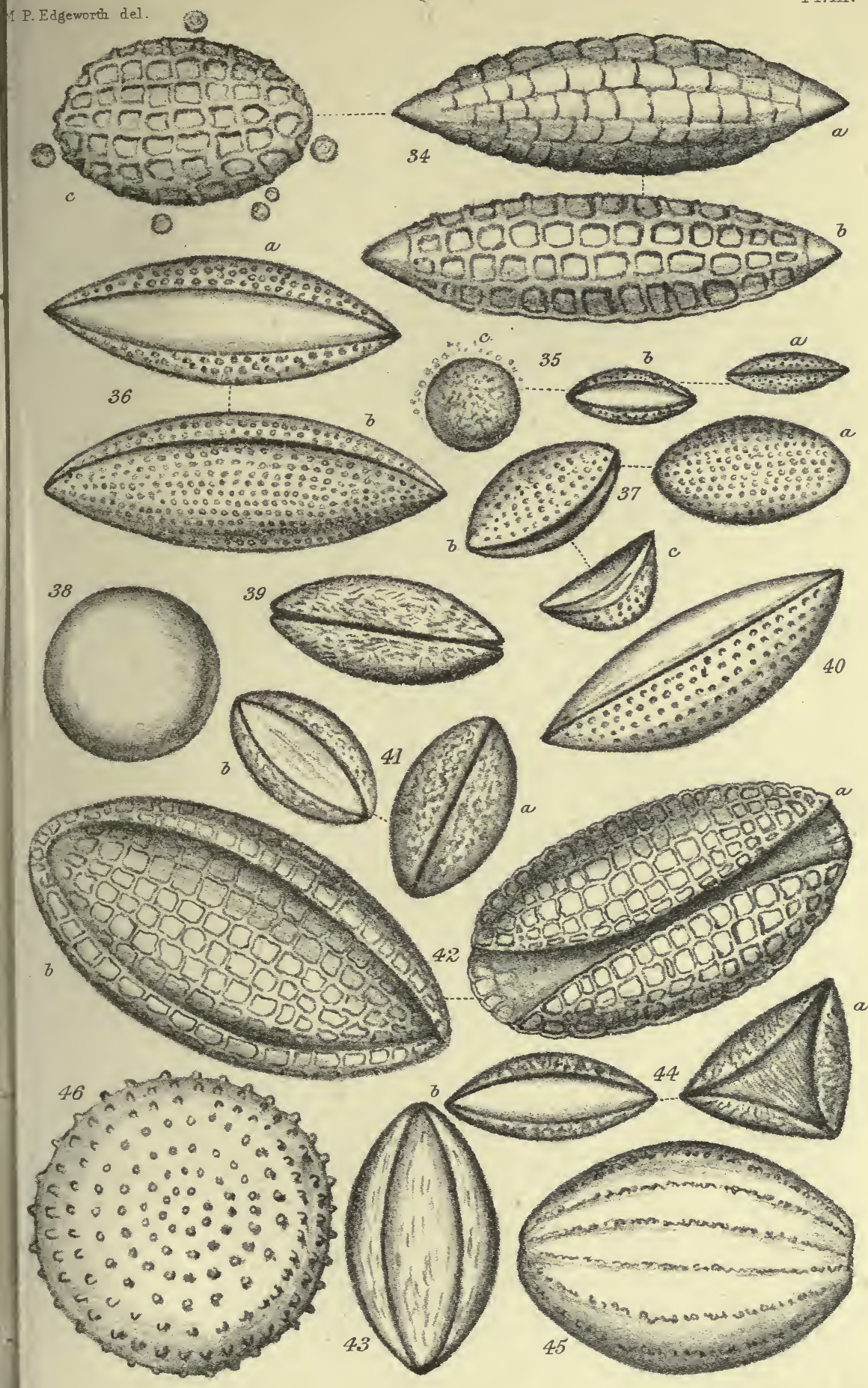

M P. Edgeworth. del.

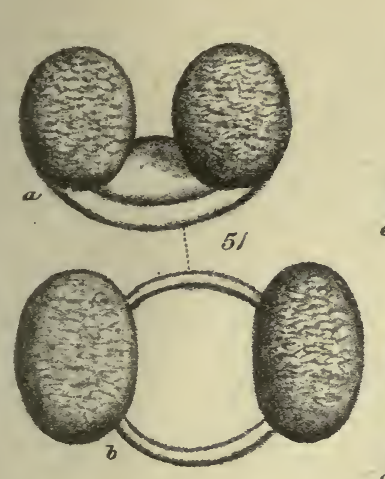

(c)

- 0 . (1)

1000

06
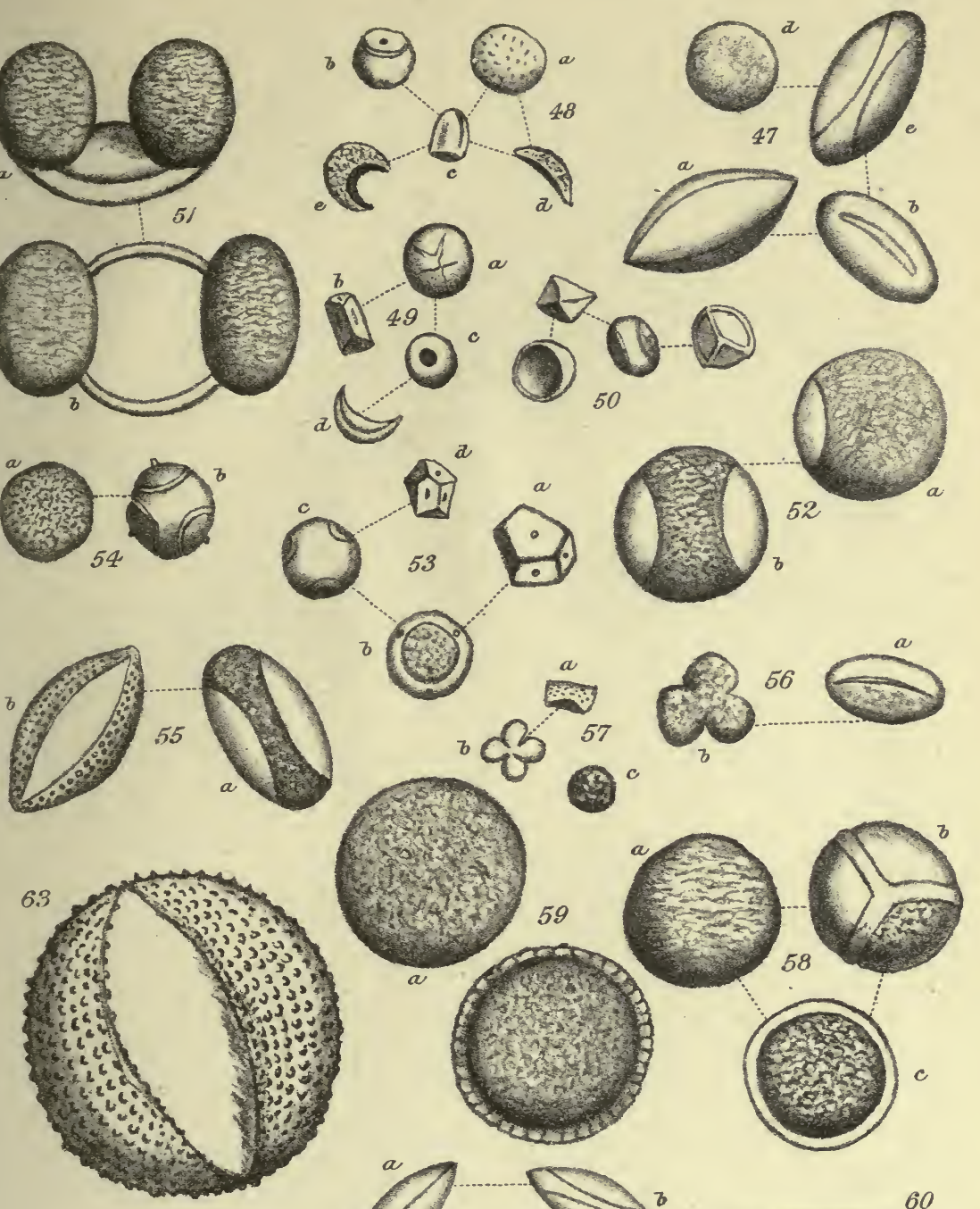

M P. Edgeworth. del.

Pl.V
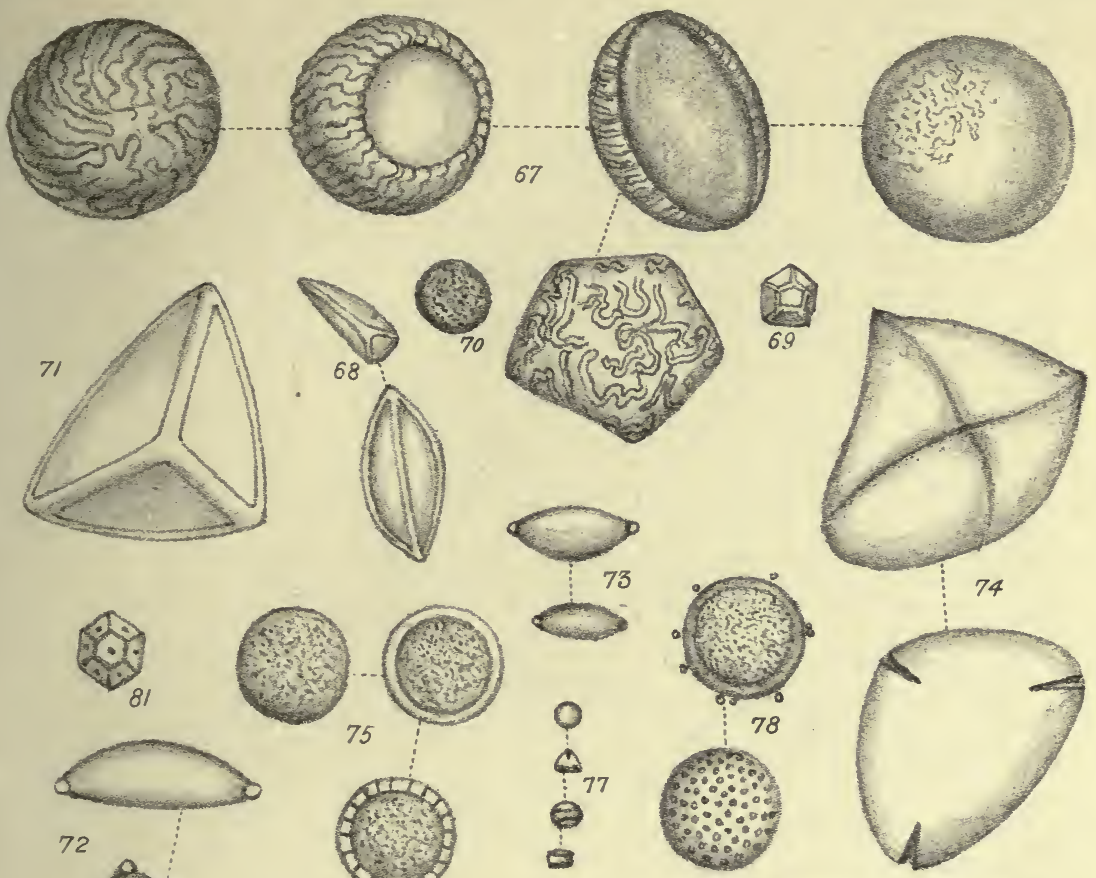

74
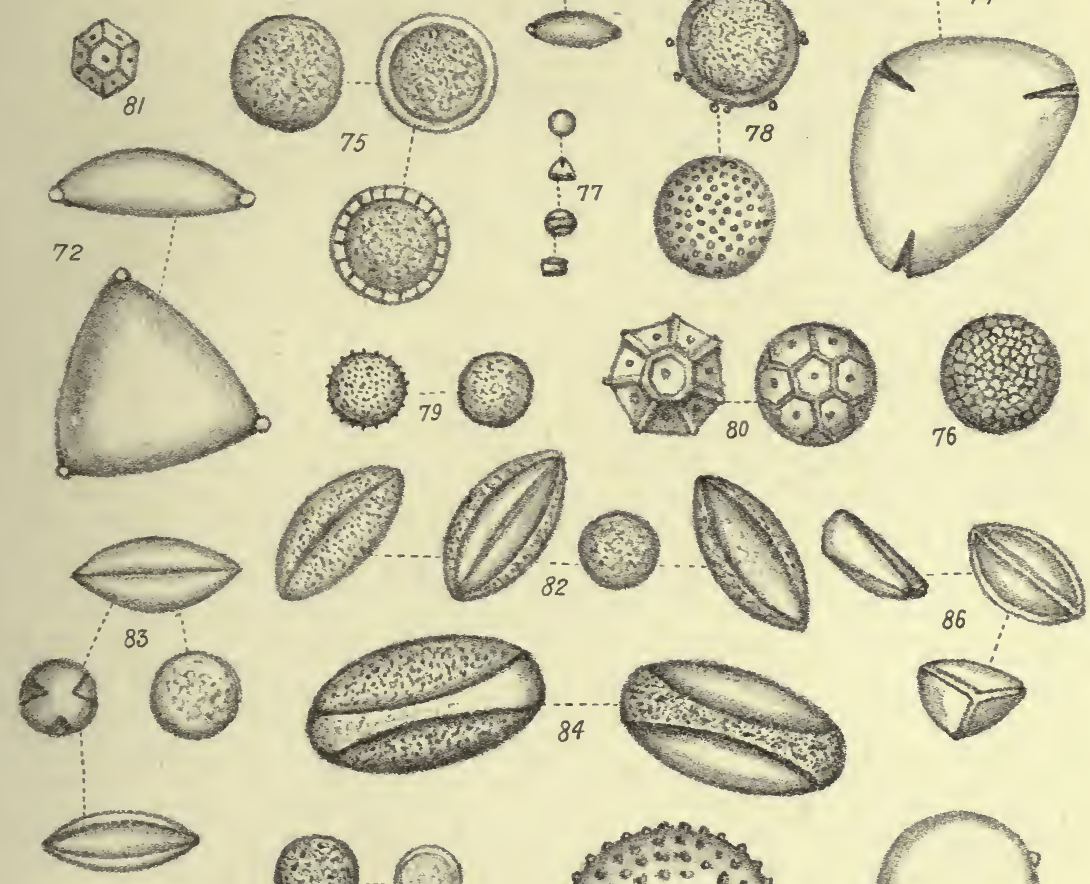
76
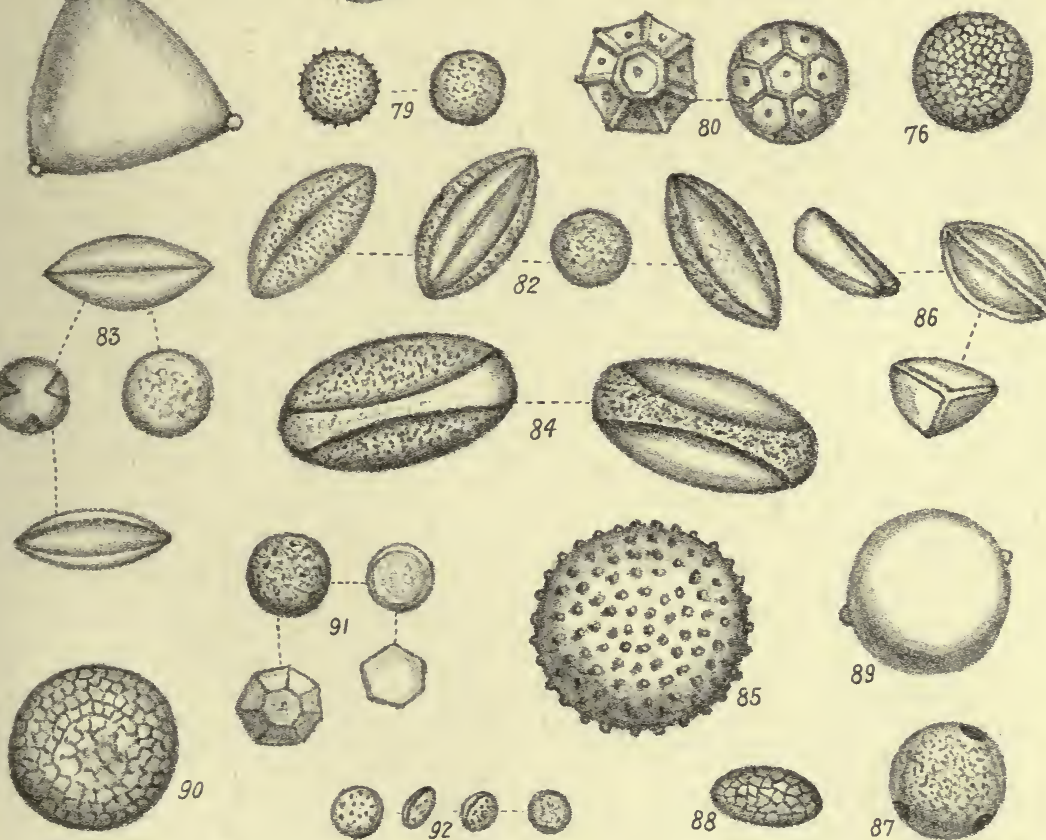
80
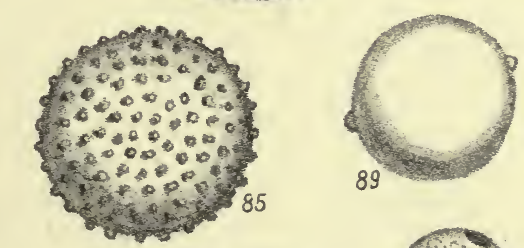

$$
\text { 웅요 }
$$

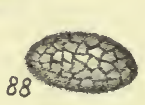

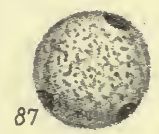

C. Berjeau. Iith. 

M.P. Edgeworth. del.

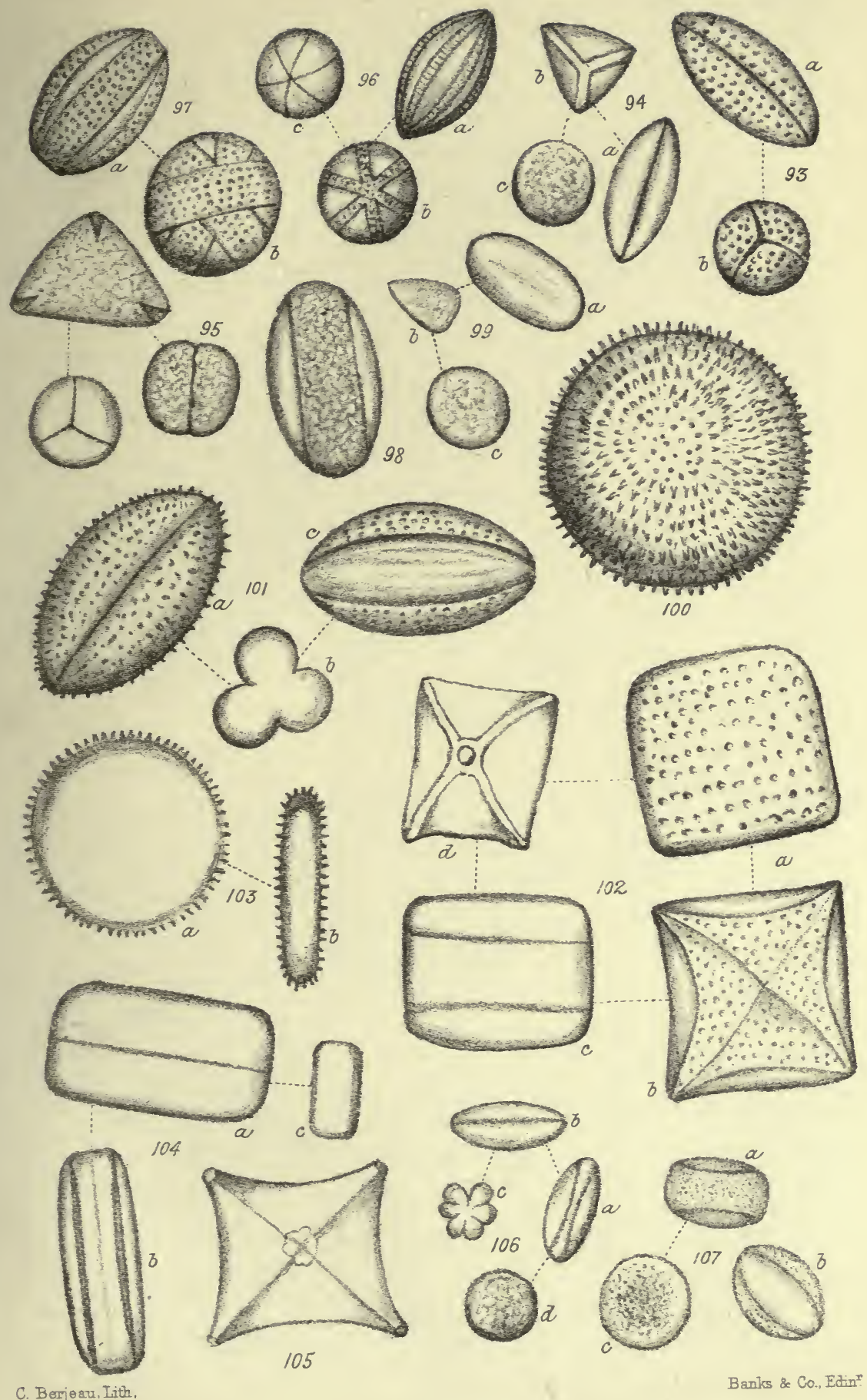




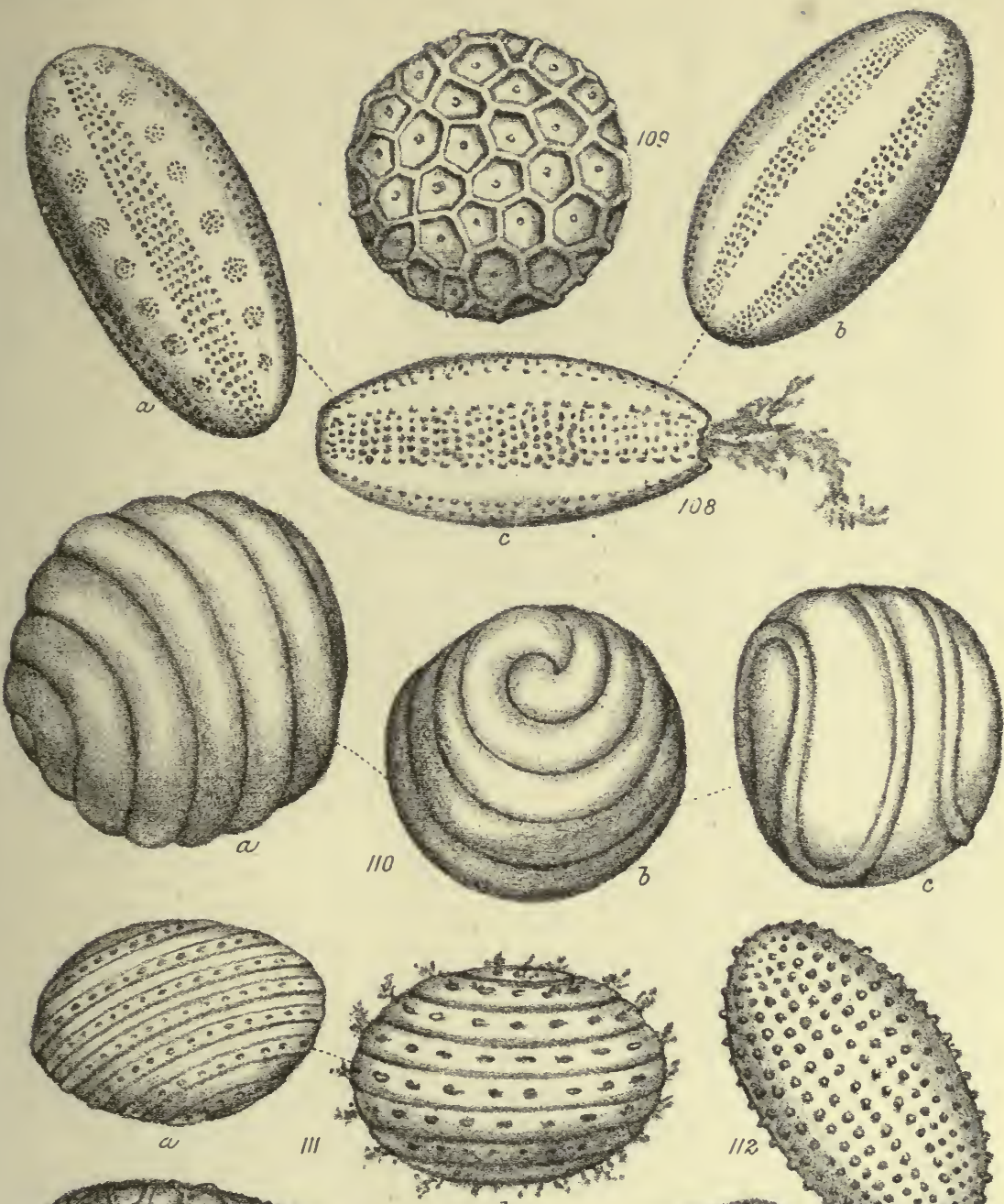
18:0\%ㅁำ $10^{\circ} \circ 0^{\circ}, 2 \%$ $1000^{\circ} \div-1$ $160 \% 00001$ $1 / 2 \alpha+0.0001$
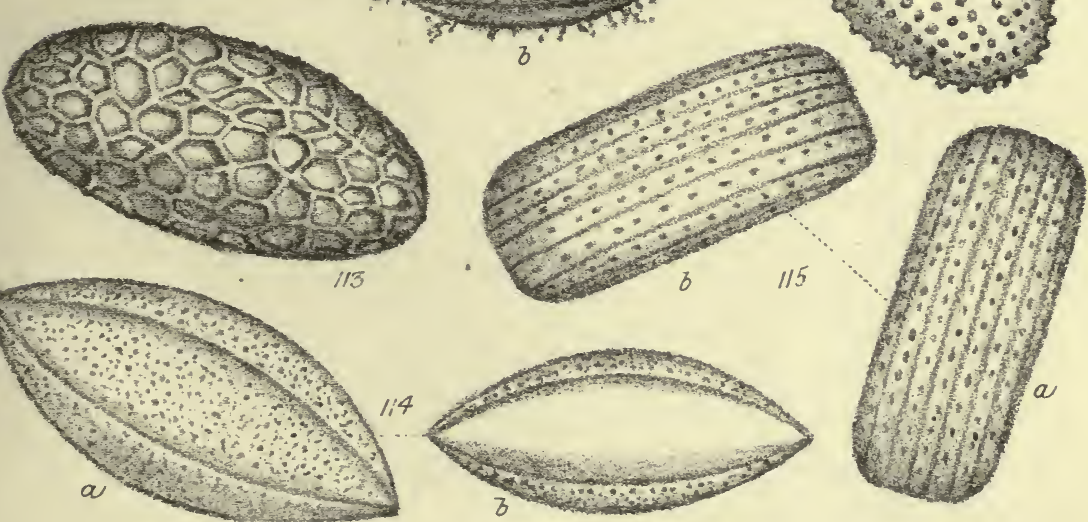

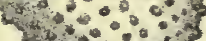

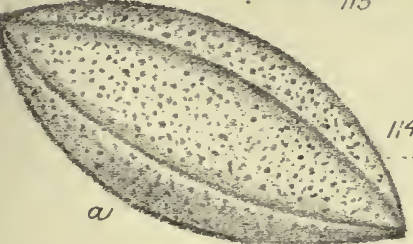

13 


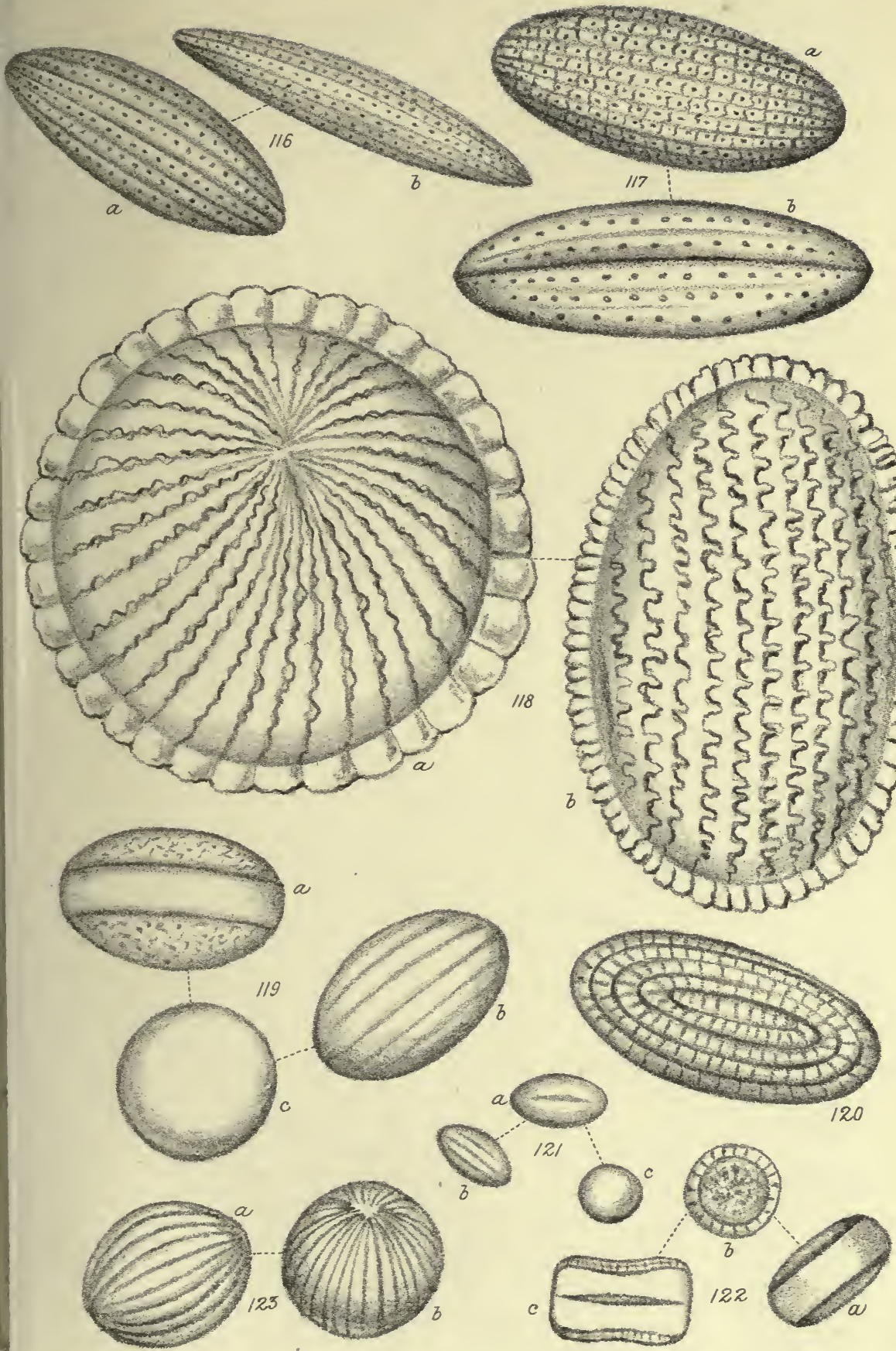

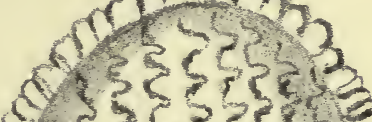

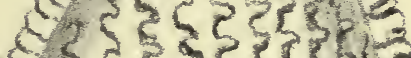

- 535535253

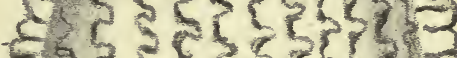

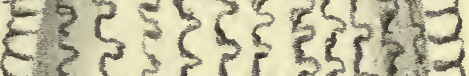

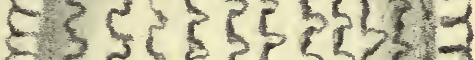

- 5 कर $5525<53$

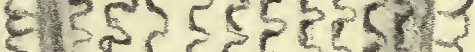

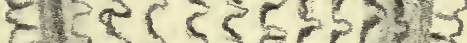

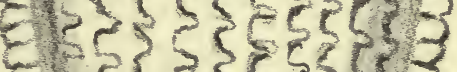

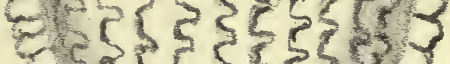

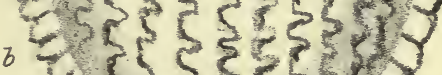

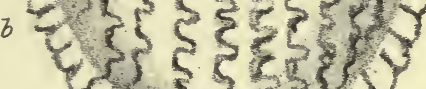




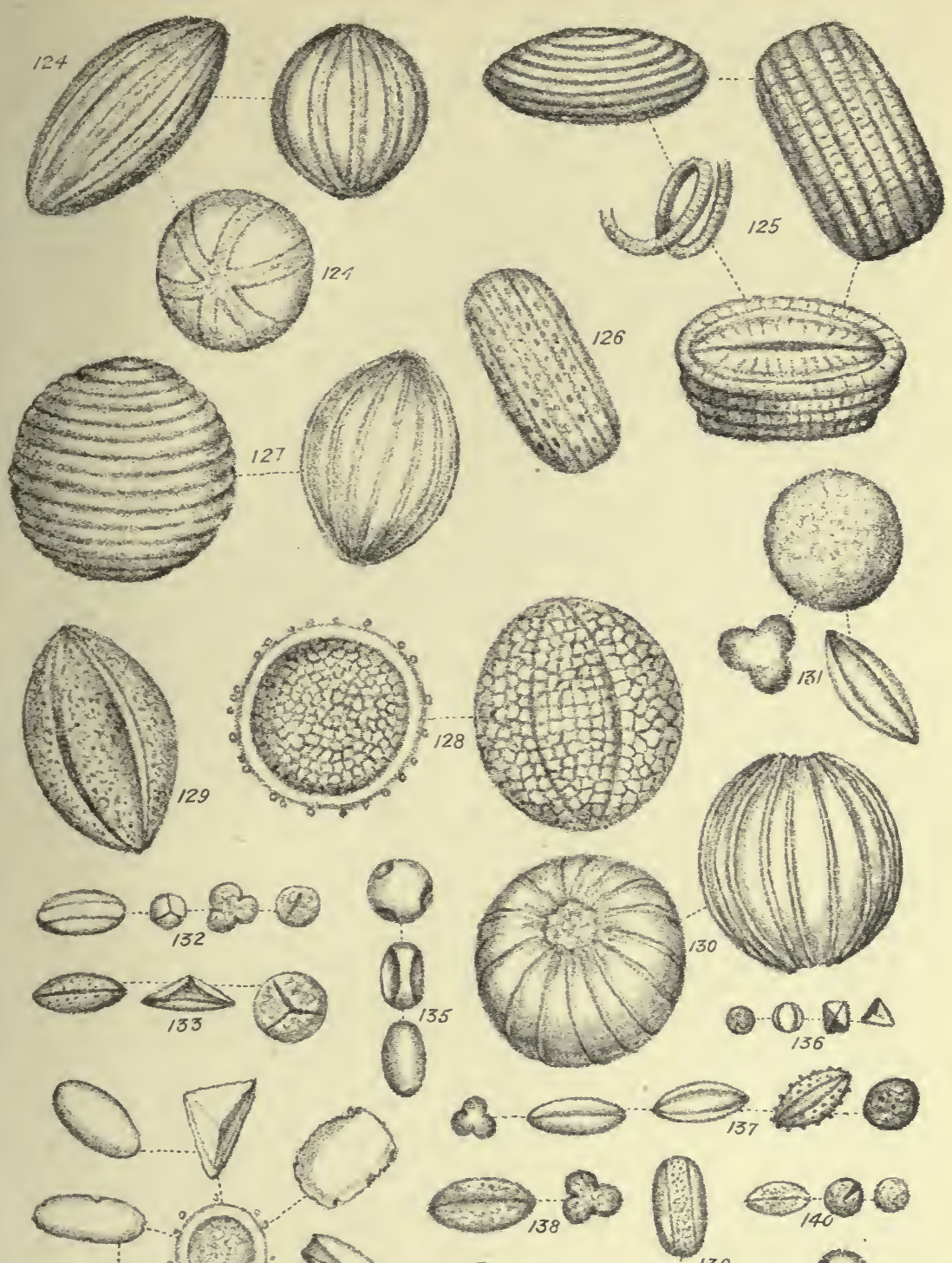
(a) ${ }_{138} \mathrm{sen} \Leftrightarrow 140$
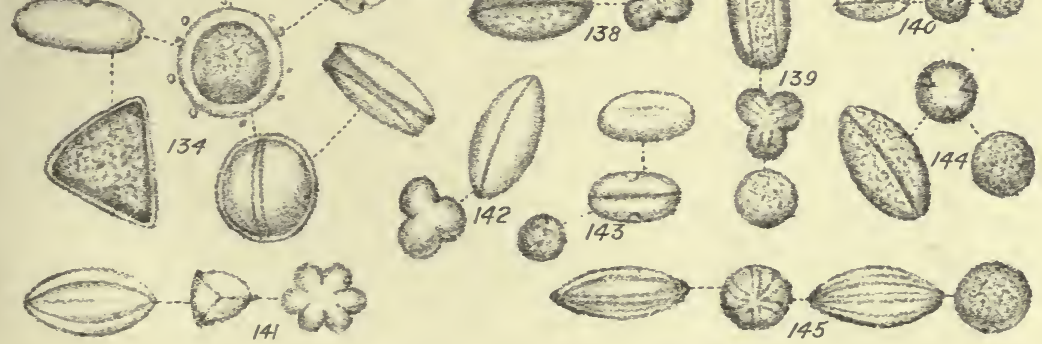



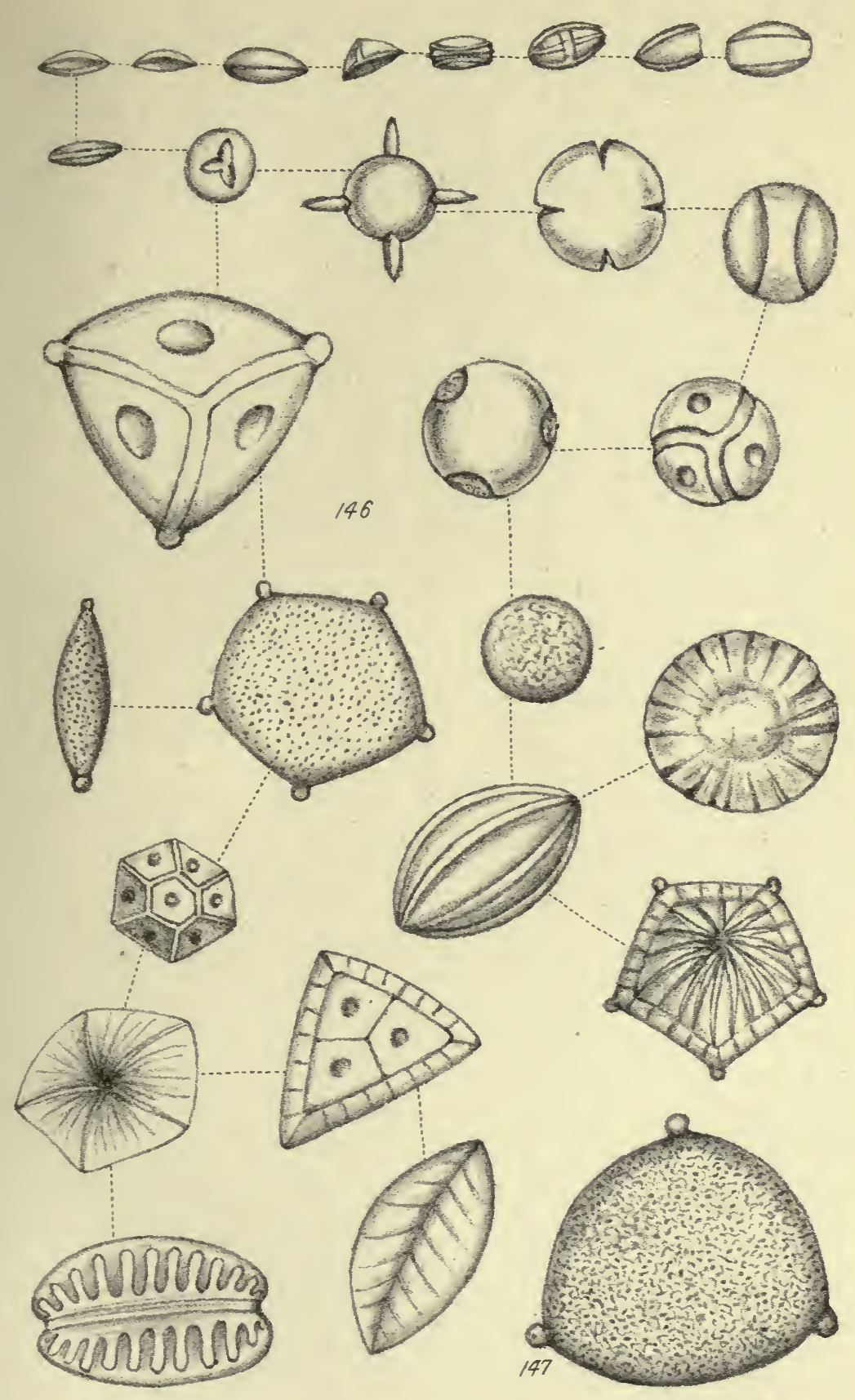

C. Berjean, Iith. 


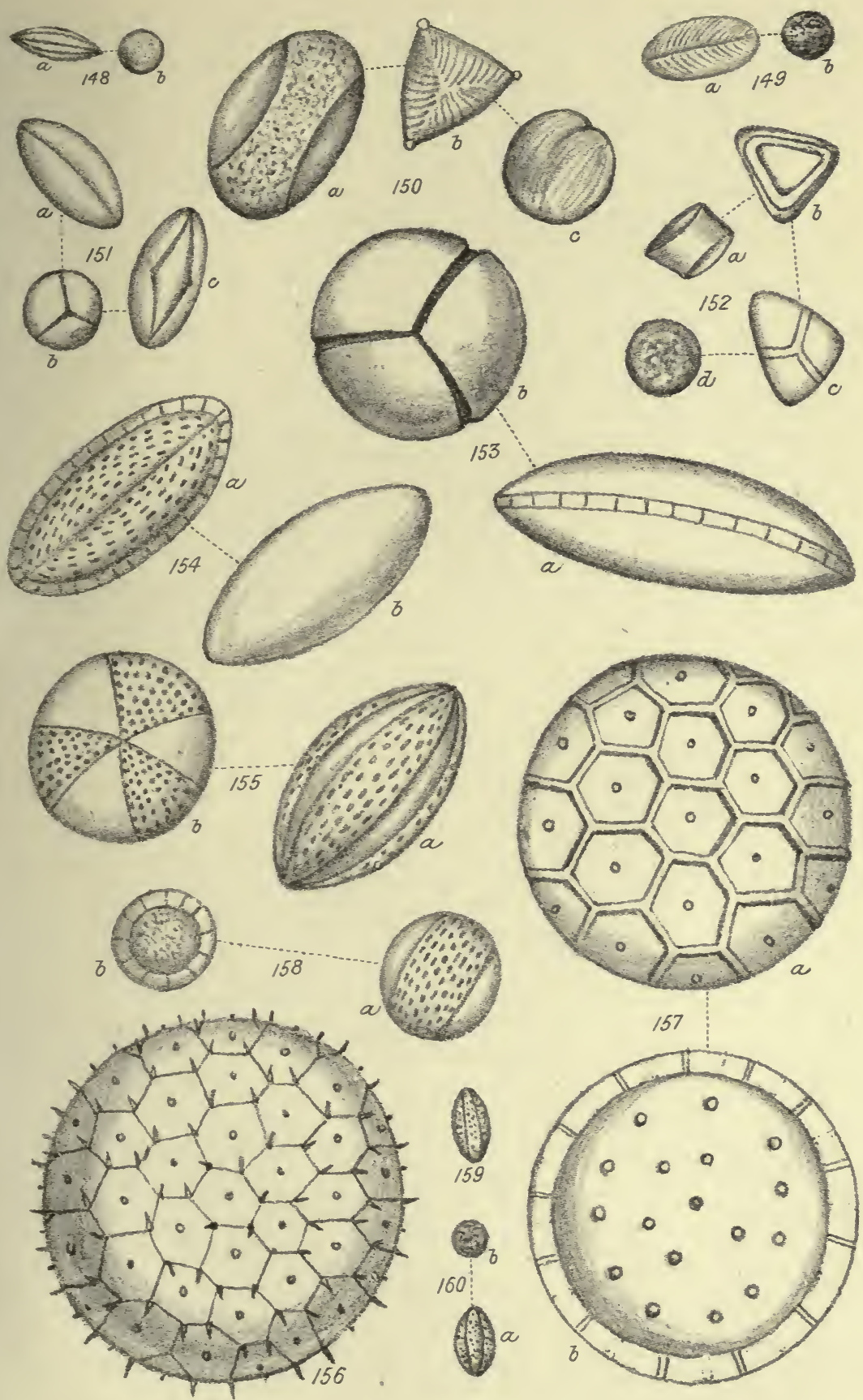



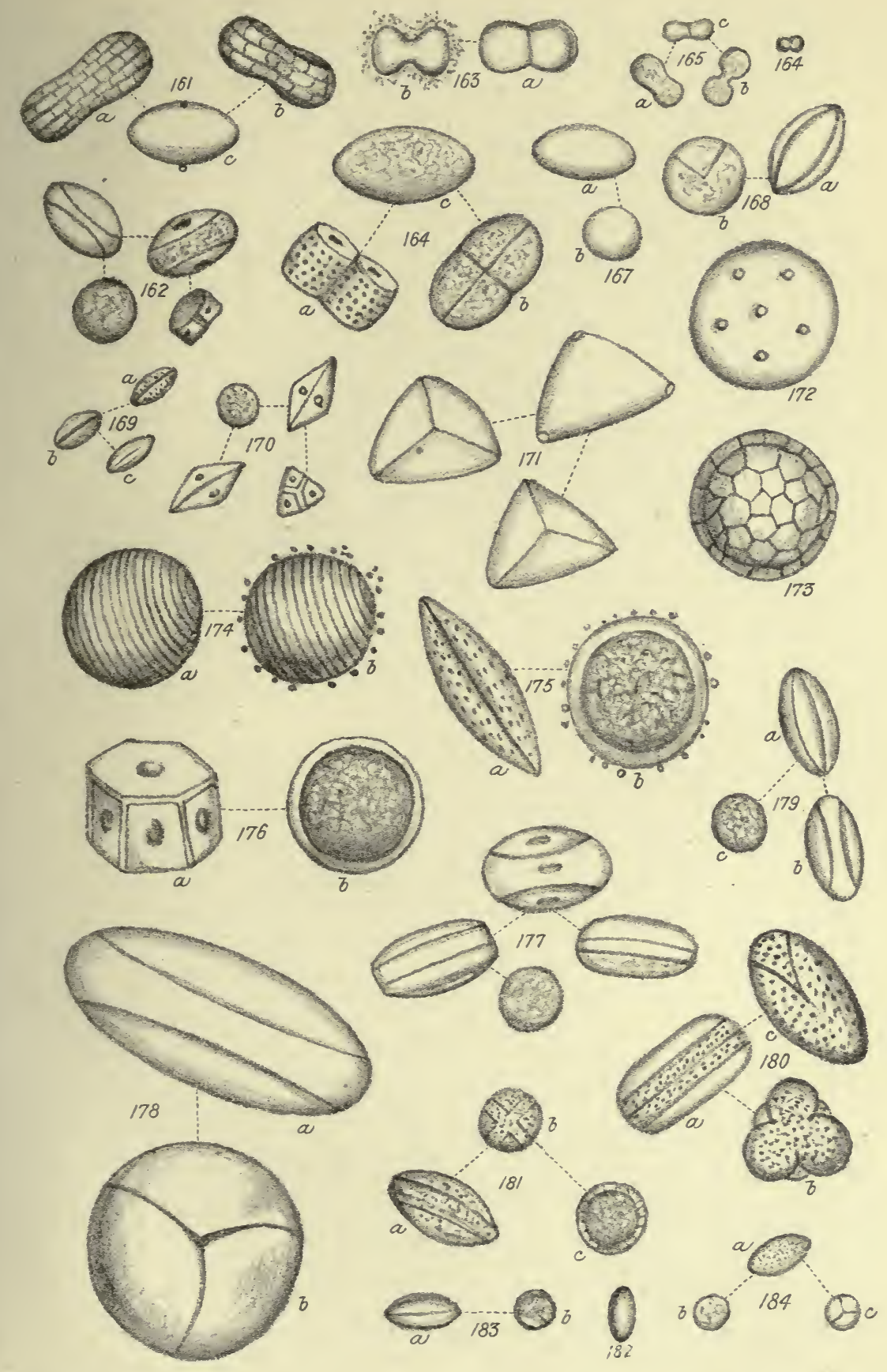





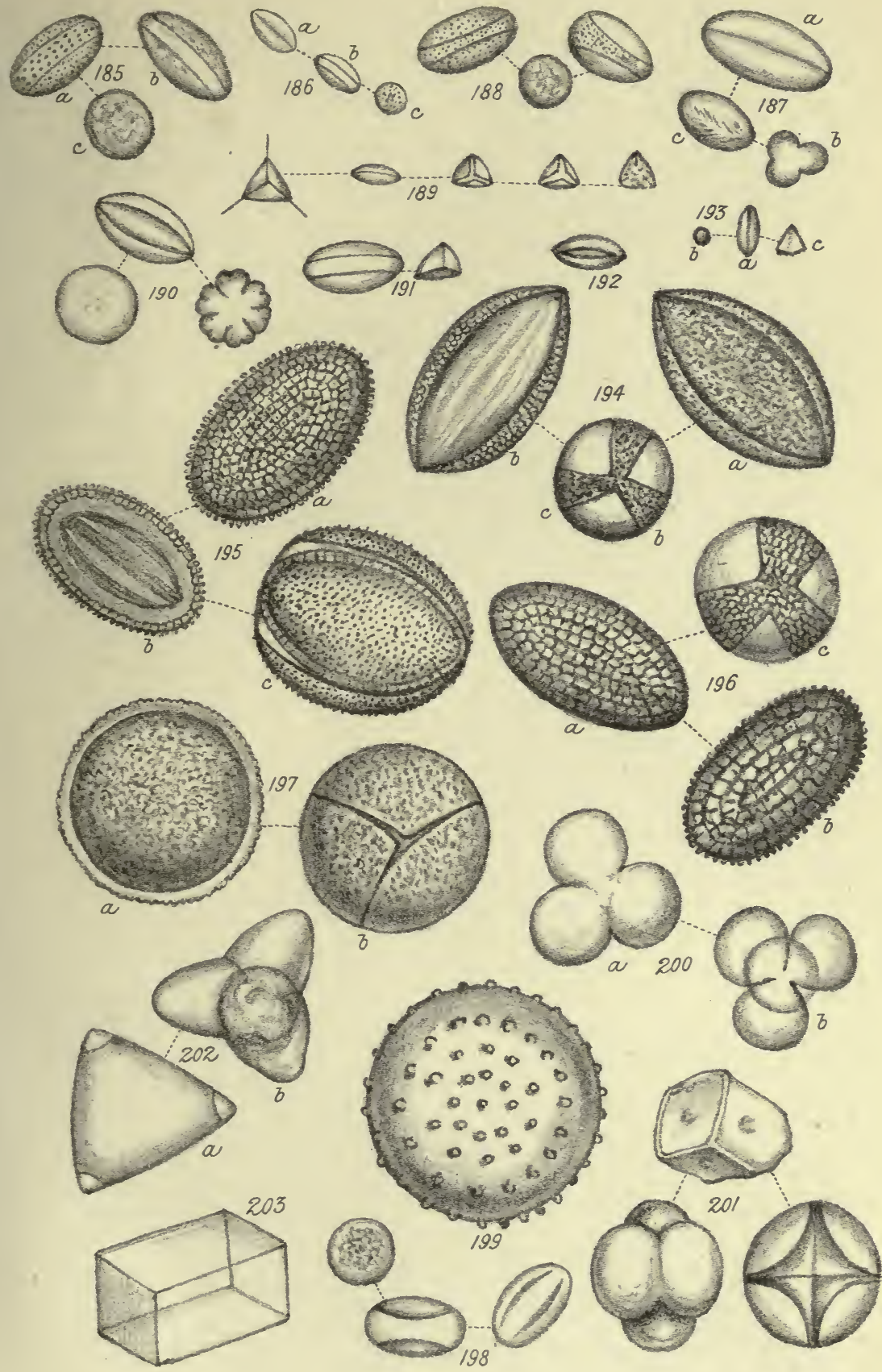




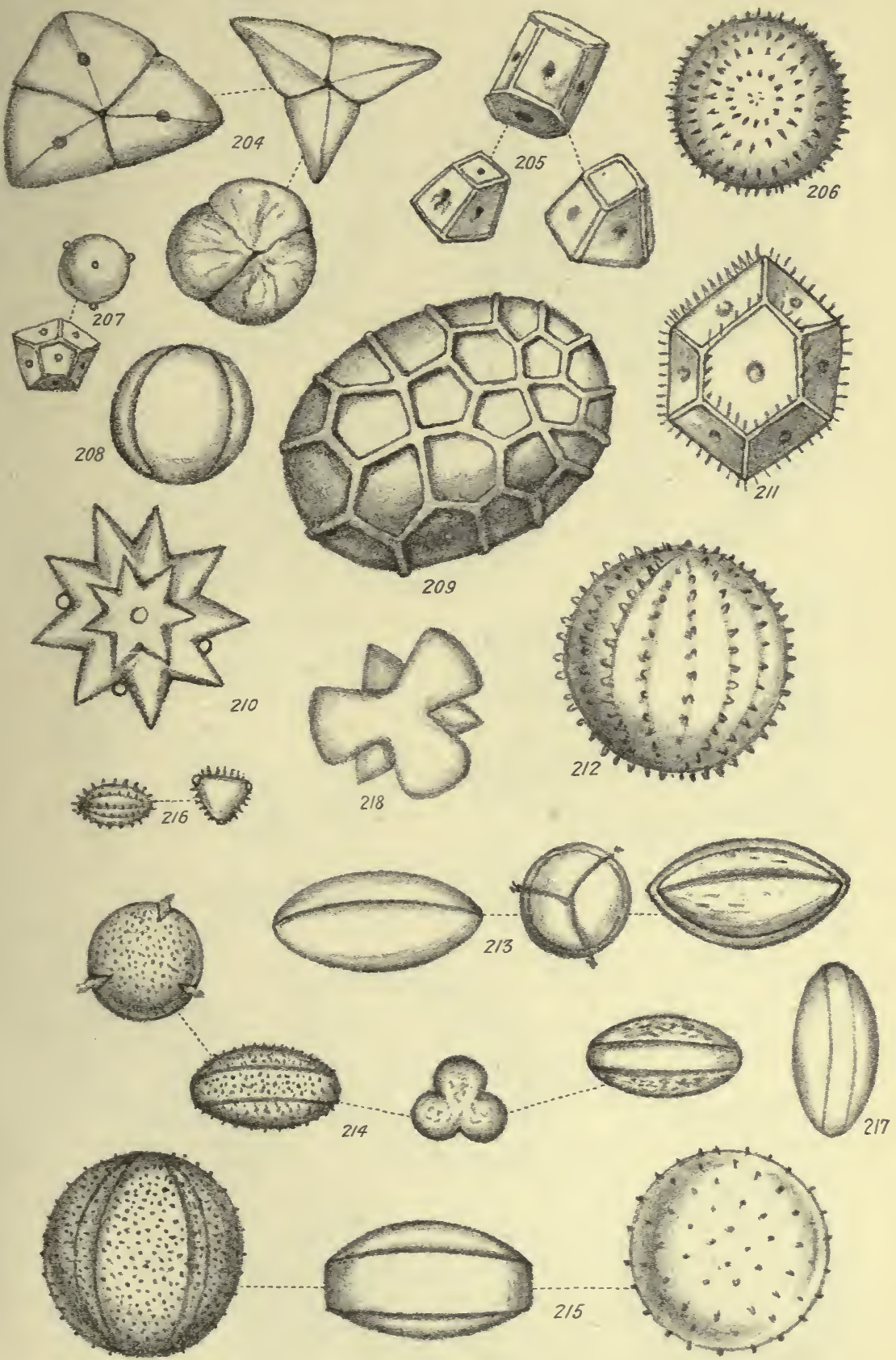


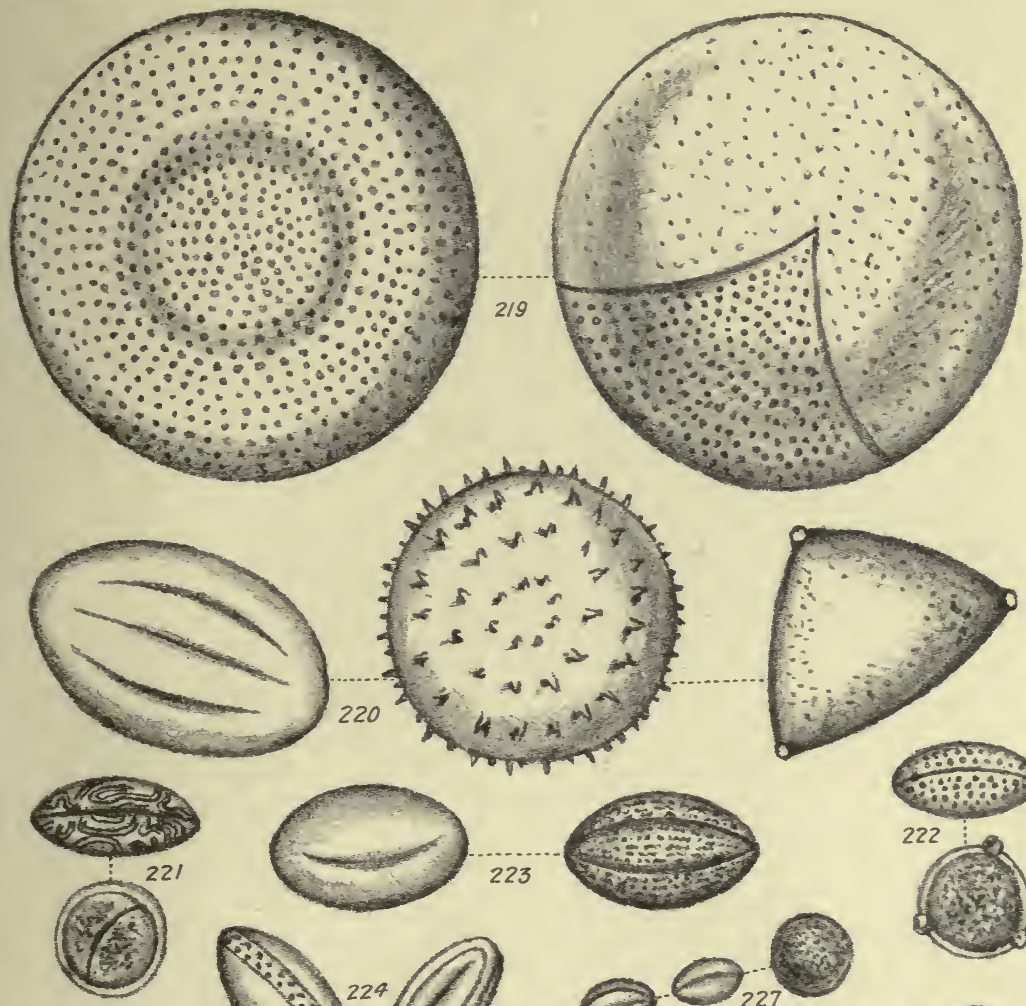

(5)
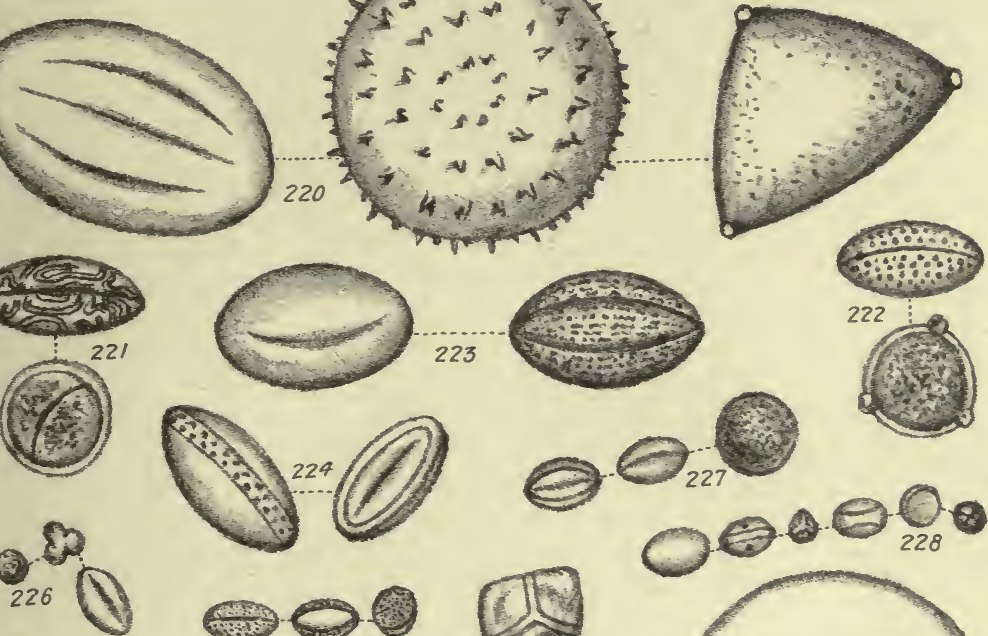

ค. 8229

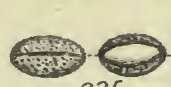

ㄱ.
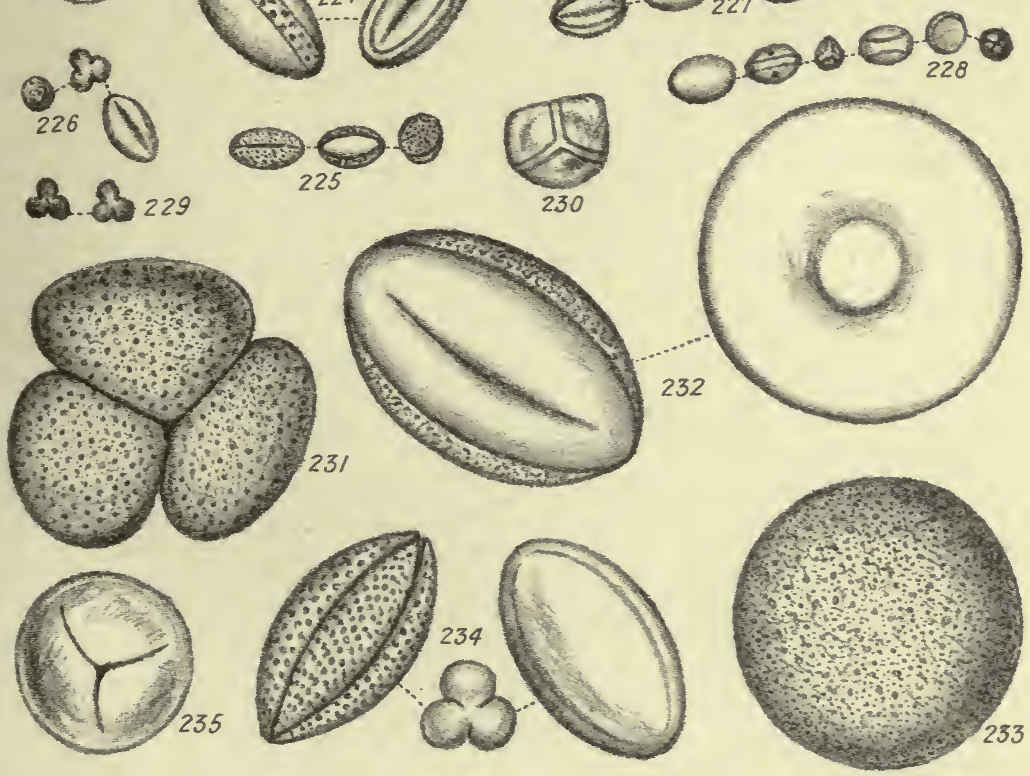

M P. Edgeworth. del.
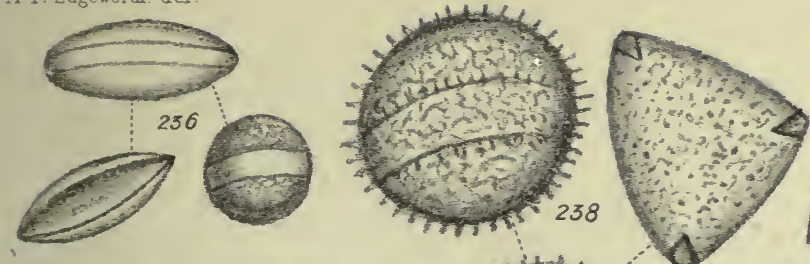

PI.XVI
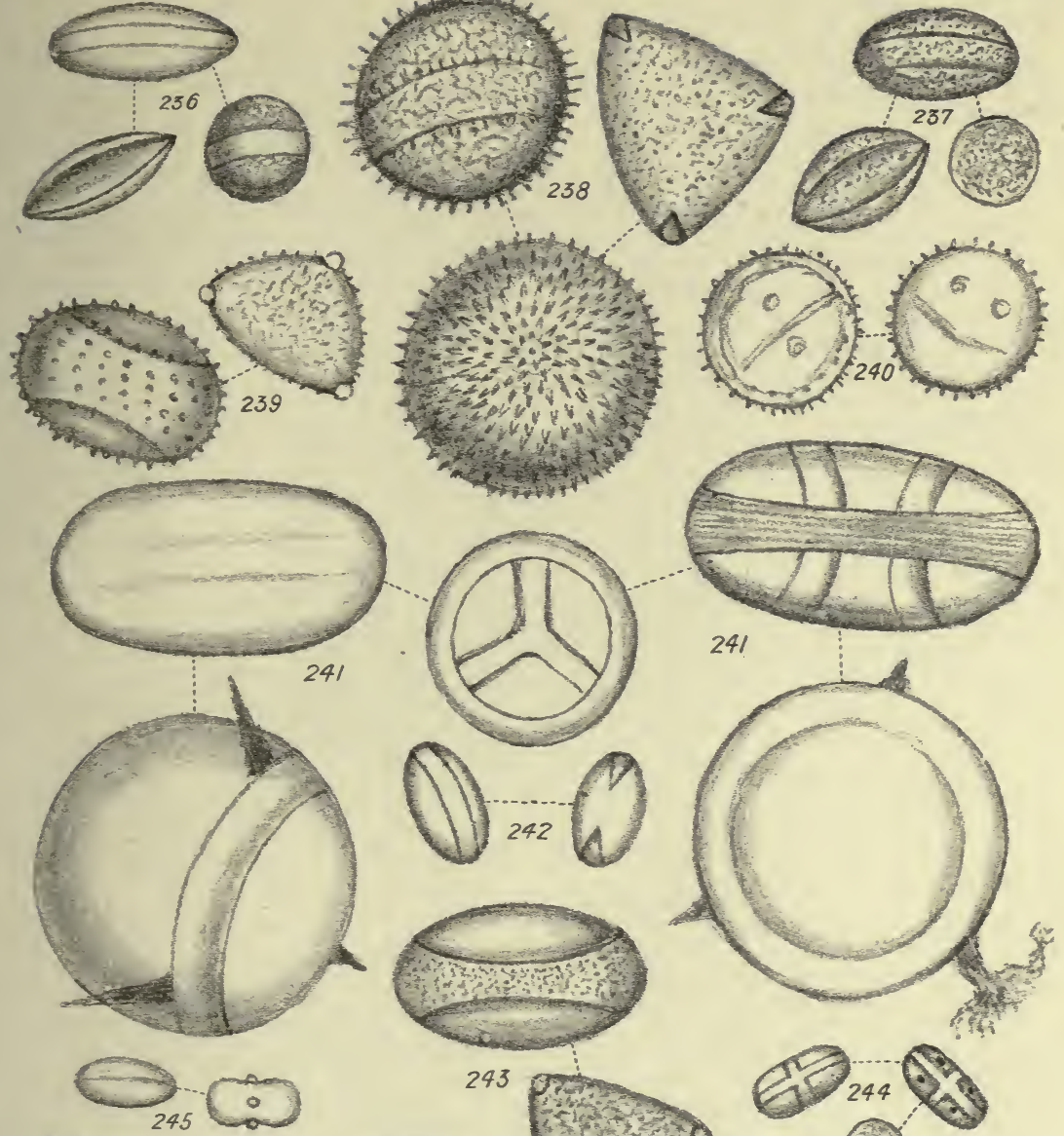

M. P. Edgeworth. del

Pl.XVII.
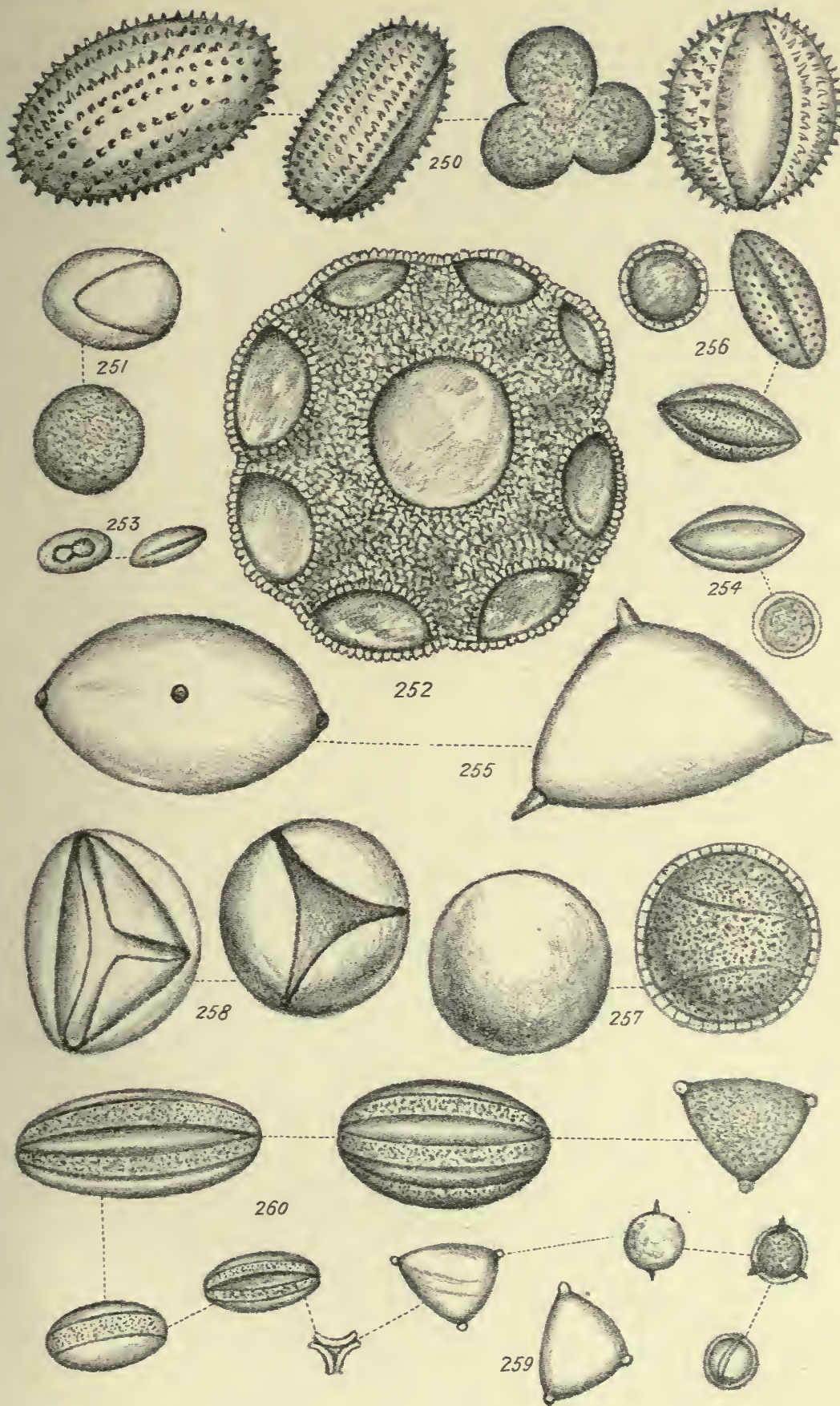

C. Berje au, Lith, 

M.P. Edgeworth. del.

PI.XVIII.
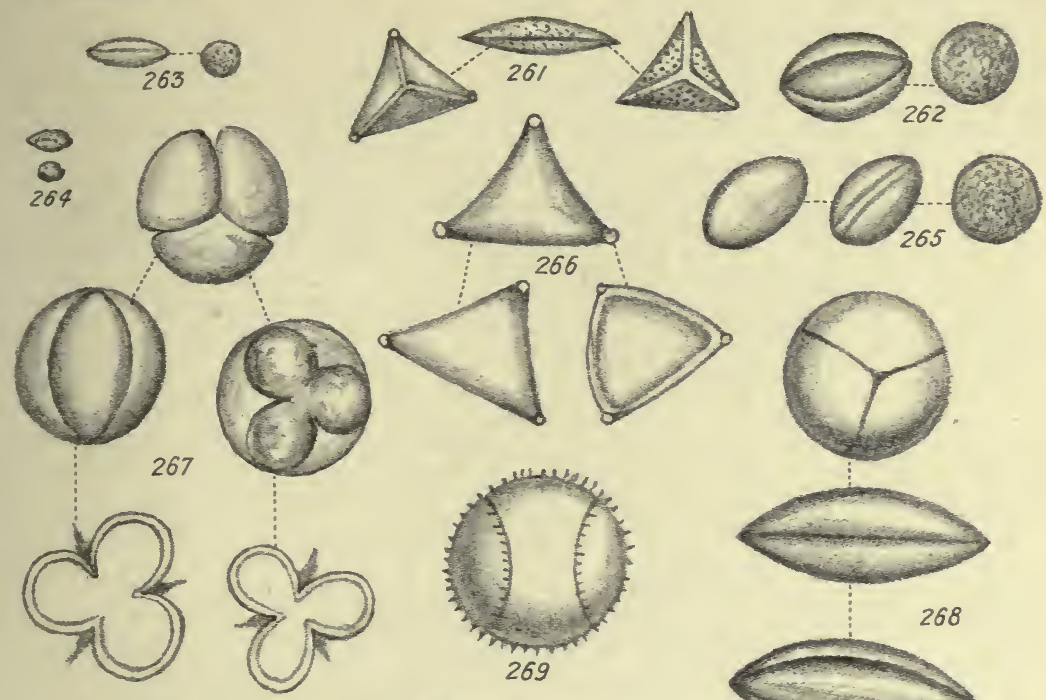

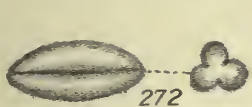

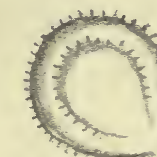

(1)
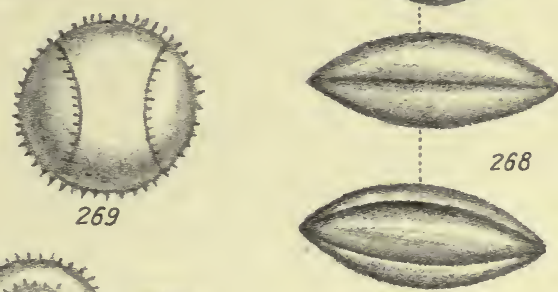

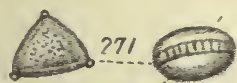

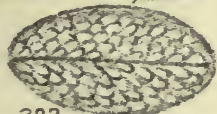

270

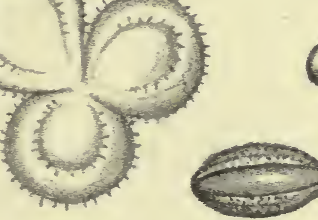

0
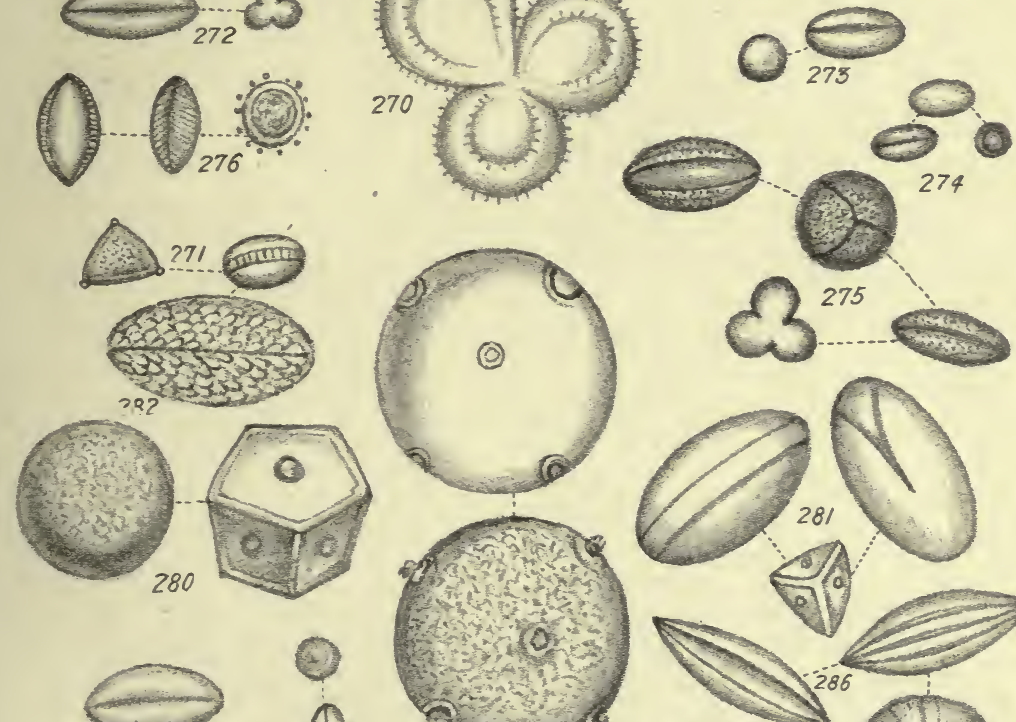

(4)

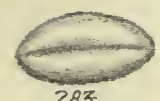

(6)
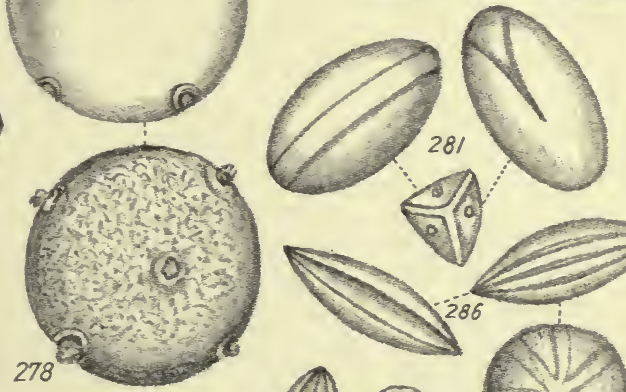

(8i.) (O1)

$0)^{285} \circ 0$
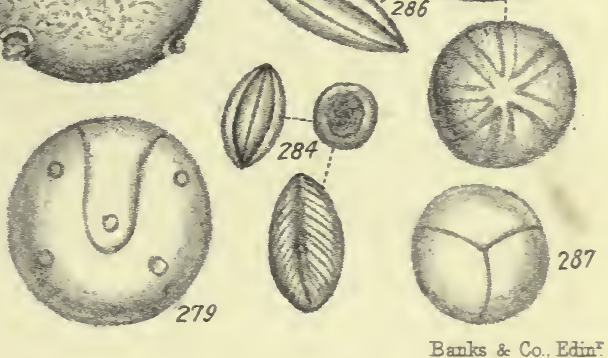


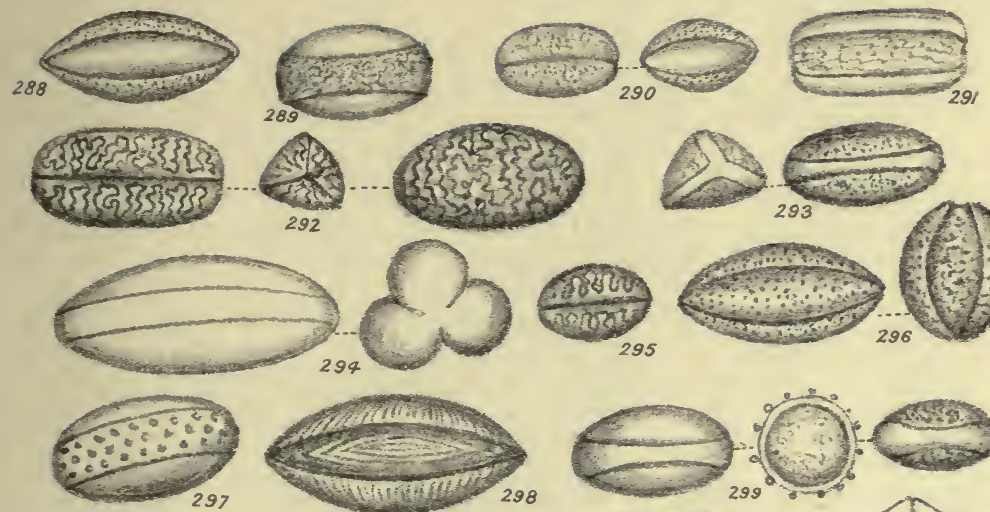

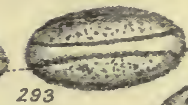
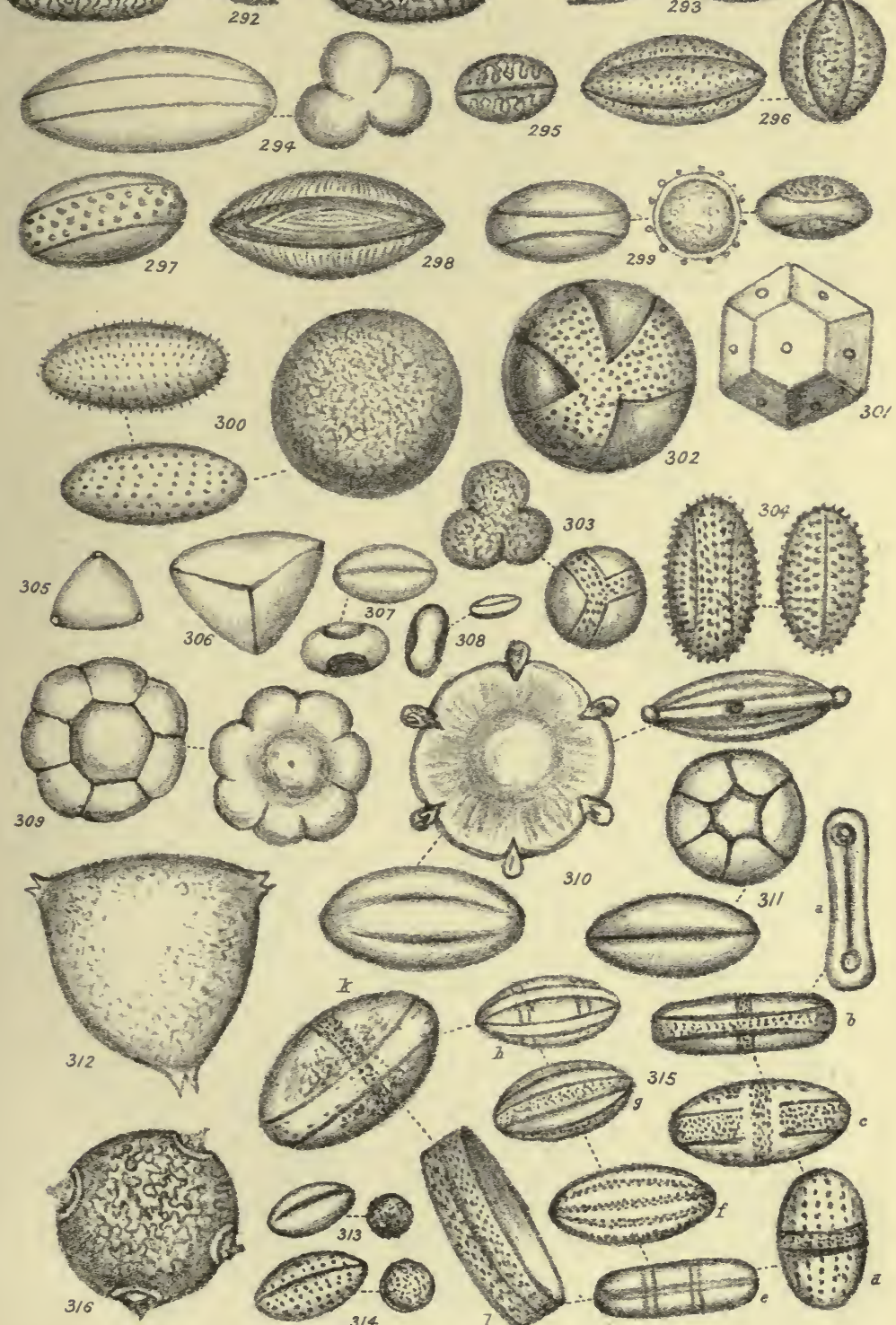

(1) 1 .
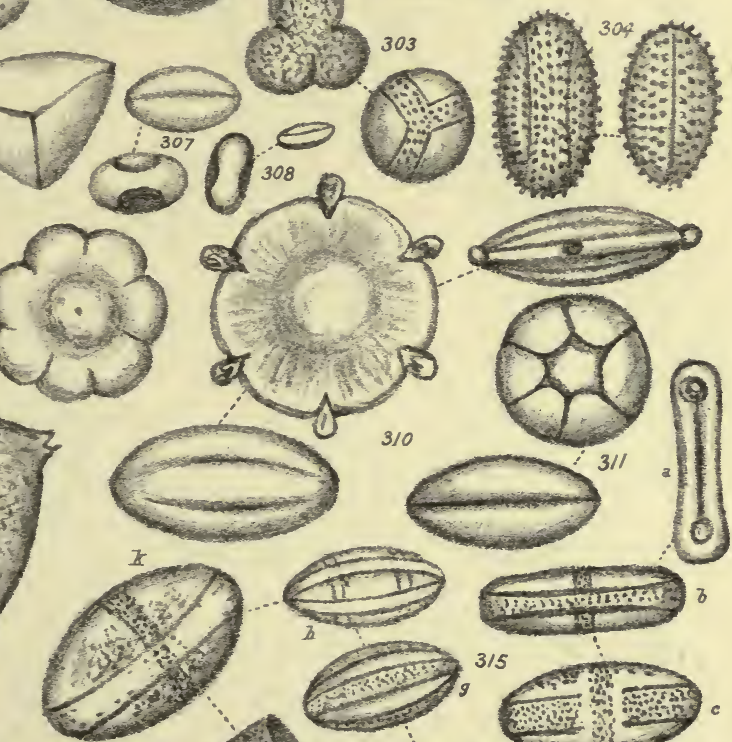

$3 / 5$
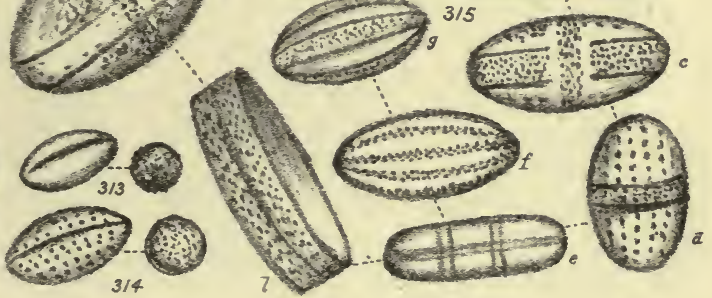


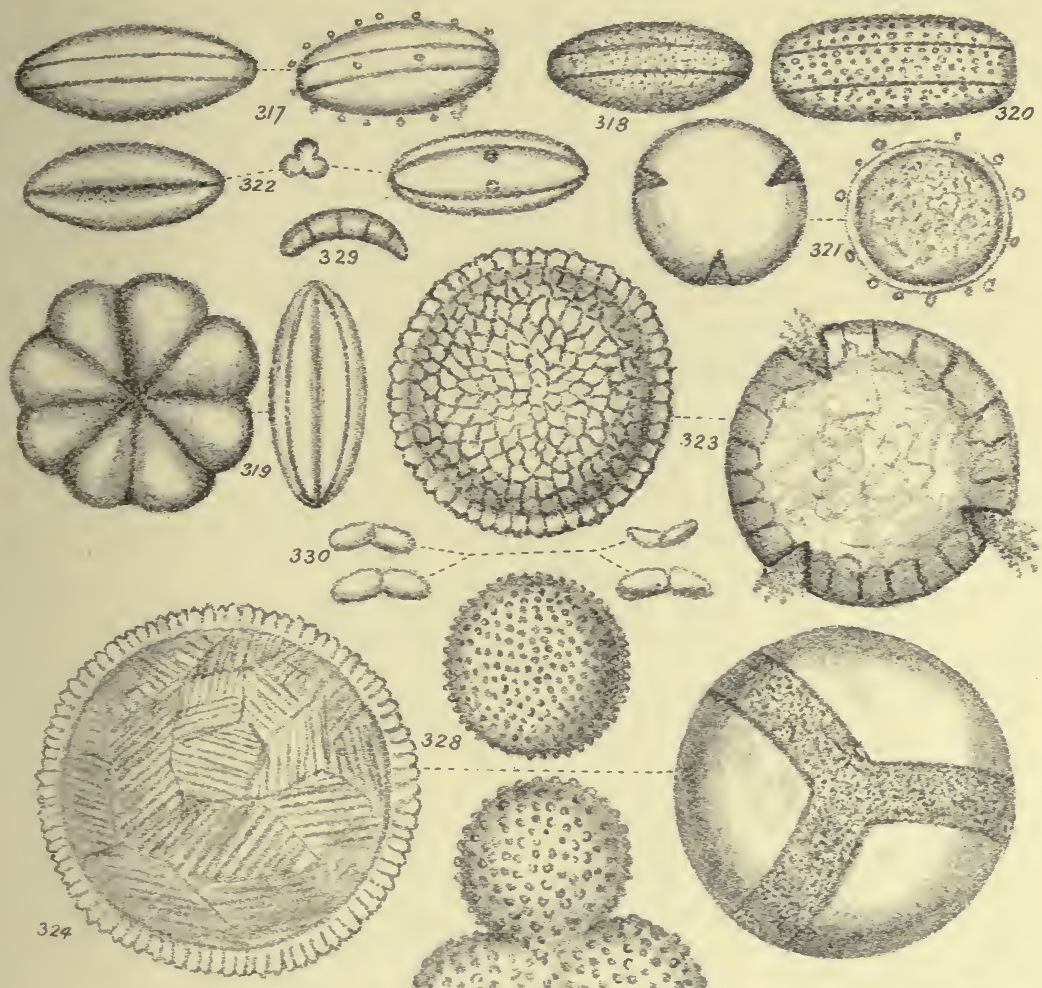
6 4 . 35 ,

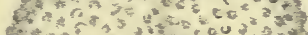

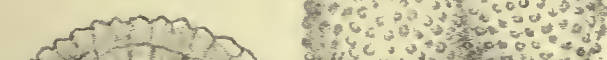
Ex)
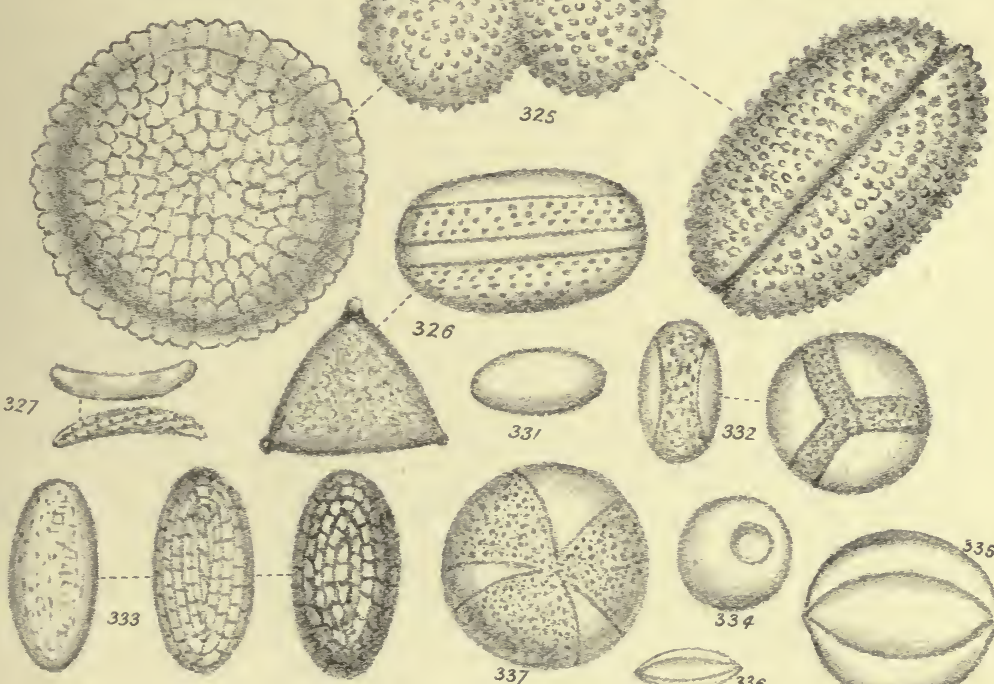
conthentwe?

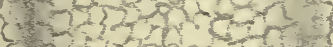
Cos?

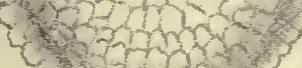
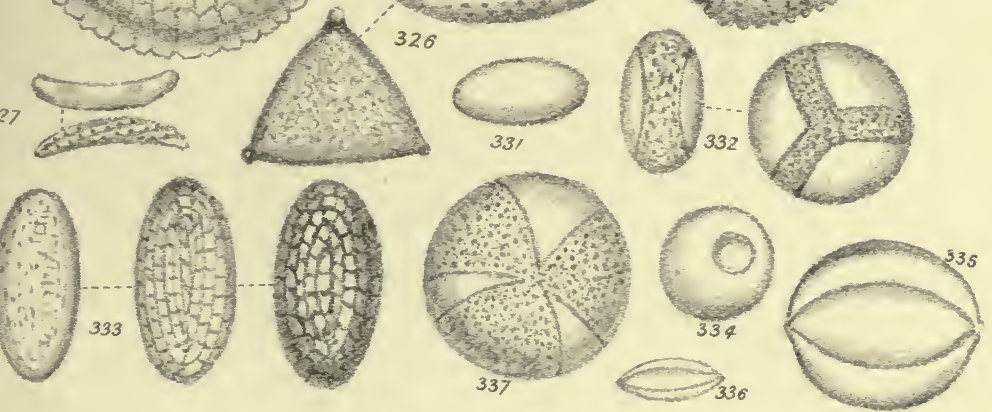


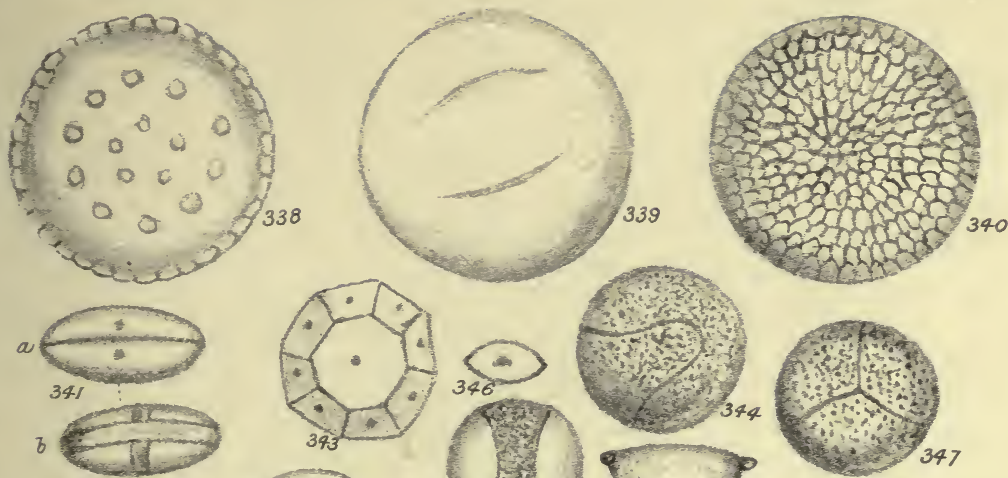

346
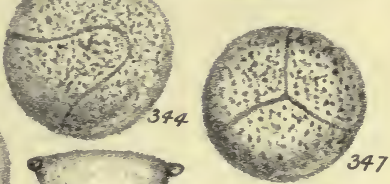

$c$ (342

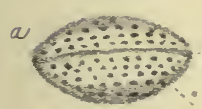
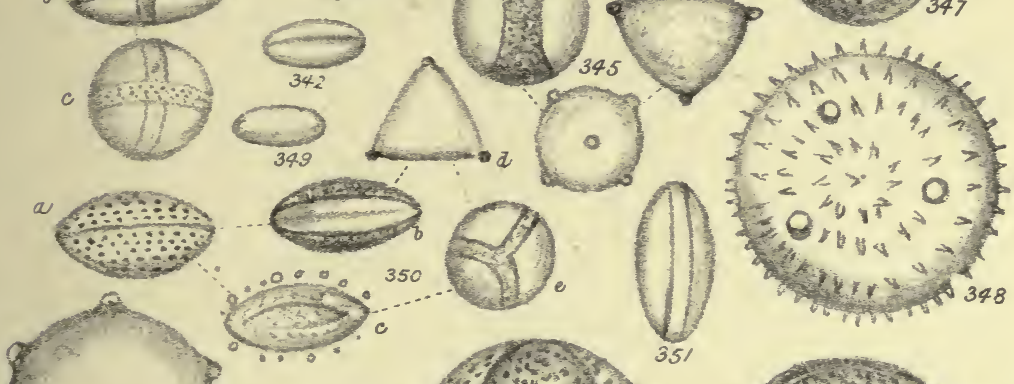

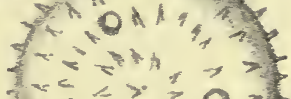
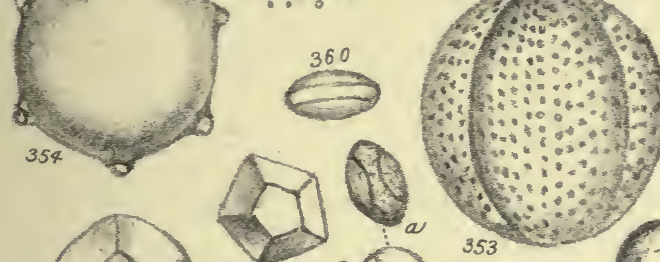

$7+<4+0=5$ $+00, b_{4}+7 k$

Wherry

\section{5 ?

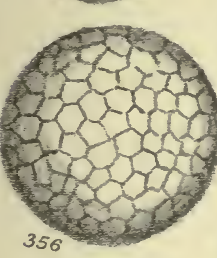

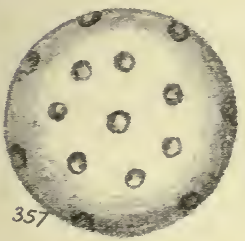
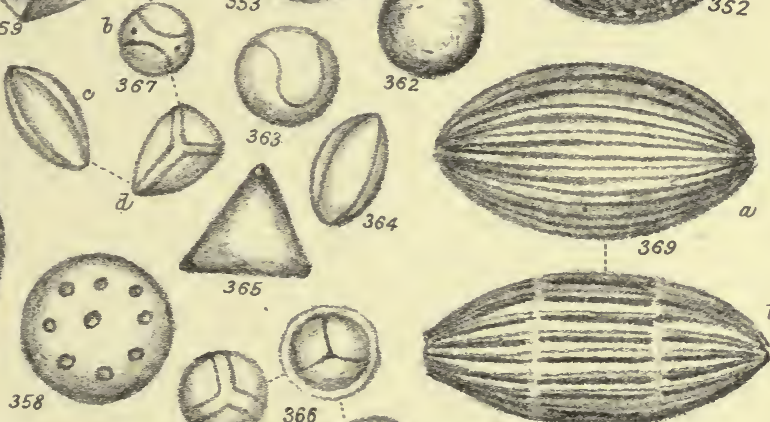

(3)

$+4,-x^{4}+x^{3}$
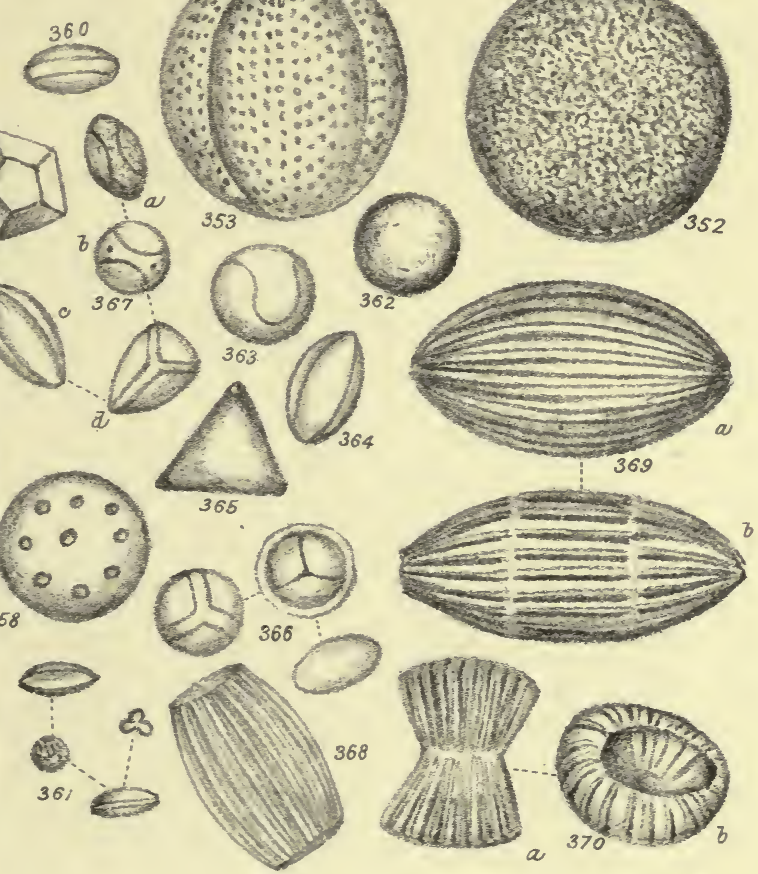

C Berjesu. Iith. 

M P. Edgeworth del.

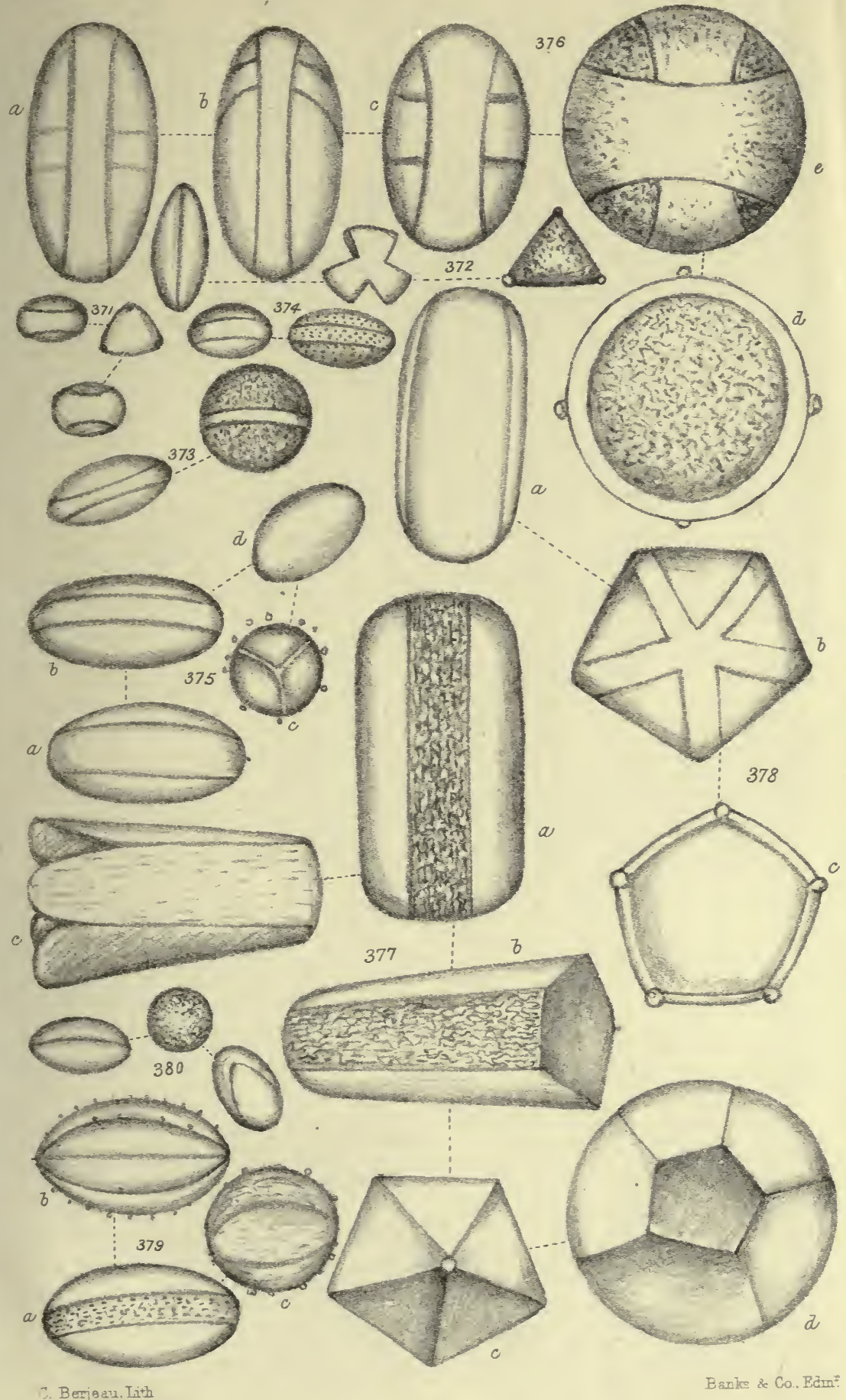



M P. Edgeworth. del.

Pl.XXIII.
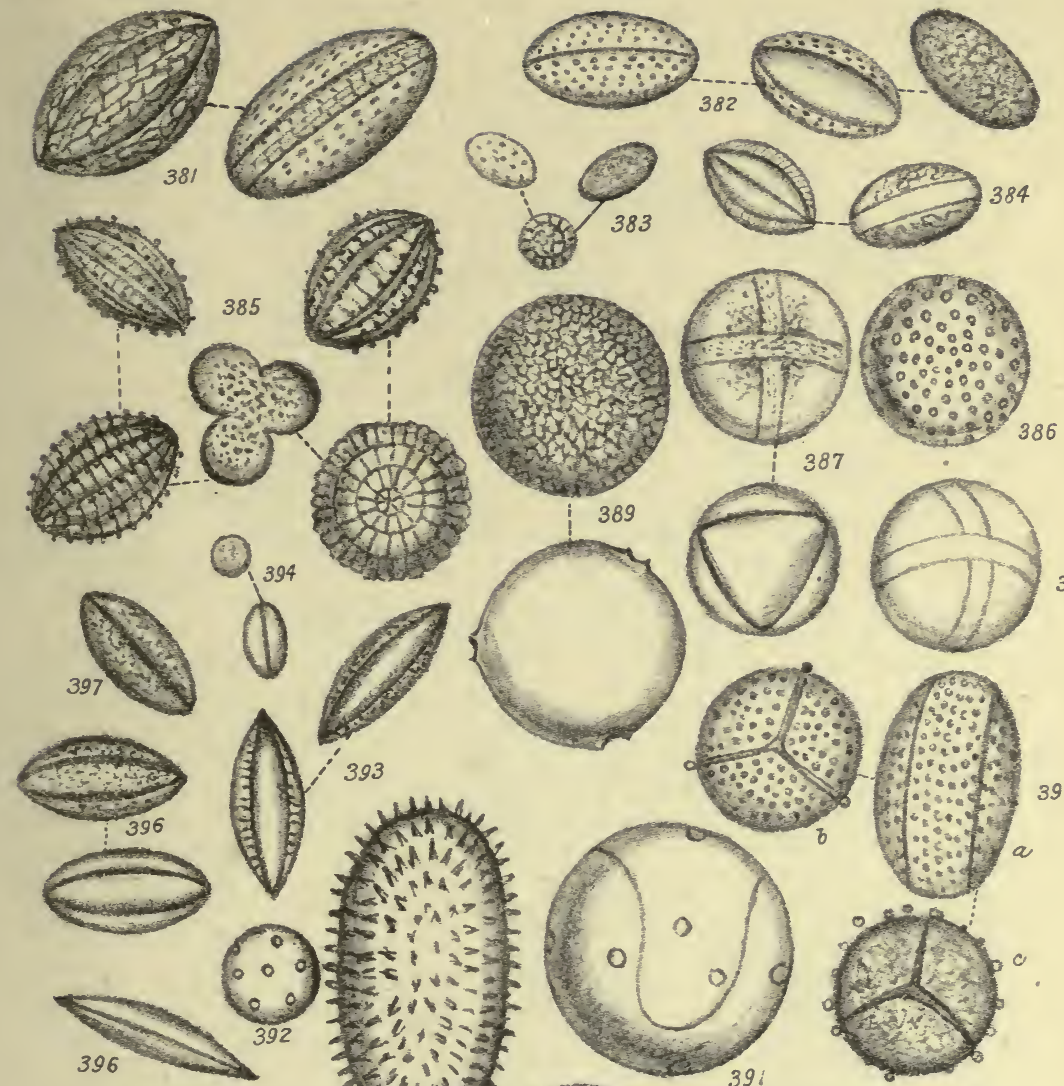

388

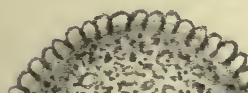

Sriction

Elyop 40013

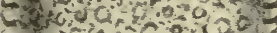

c.8.0.

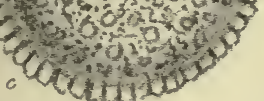

$\therefore 393$

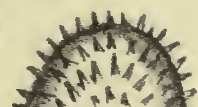

$+\mathrm{N}^{2}+\mathrm{H}^{2}$

$x_{-\infty}+a x>=6$

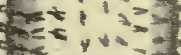

$x^{2}+6+2=3$

$6,4,3^{2}=36$

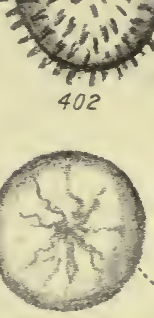

399
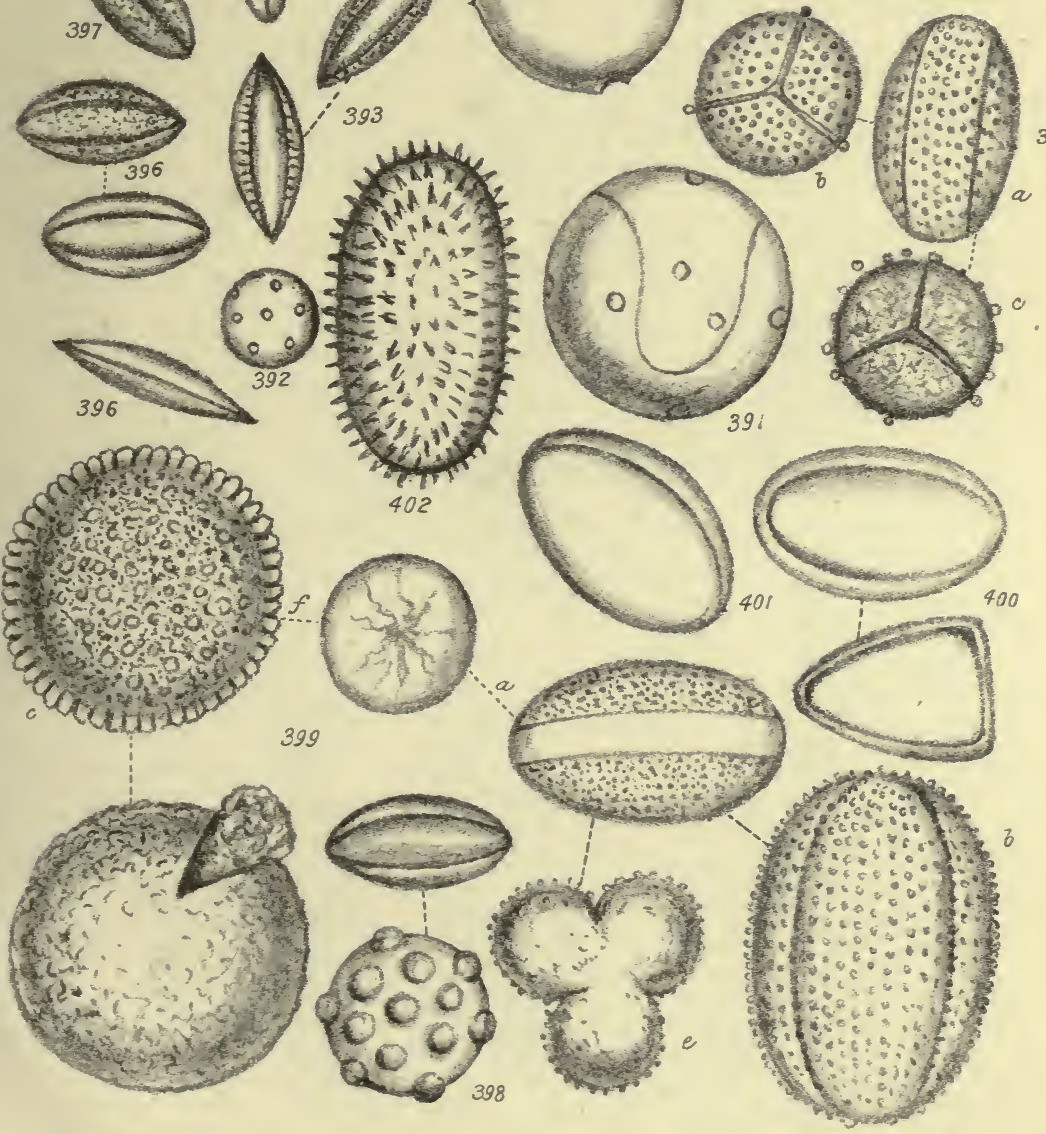

C. Berjeau. Lith. 

M. P. Edgeworth. del.

P1.XXIV
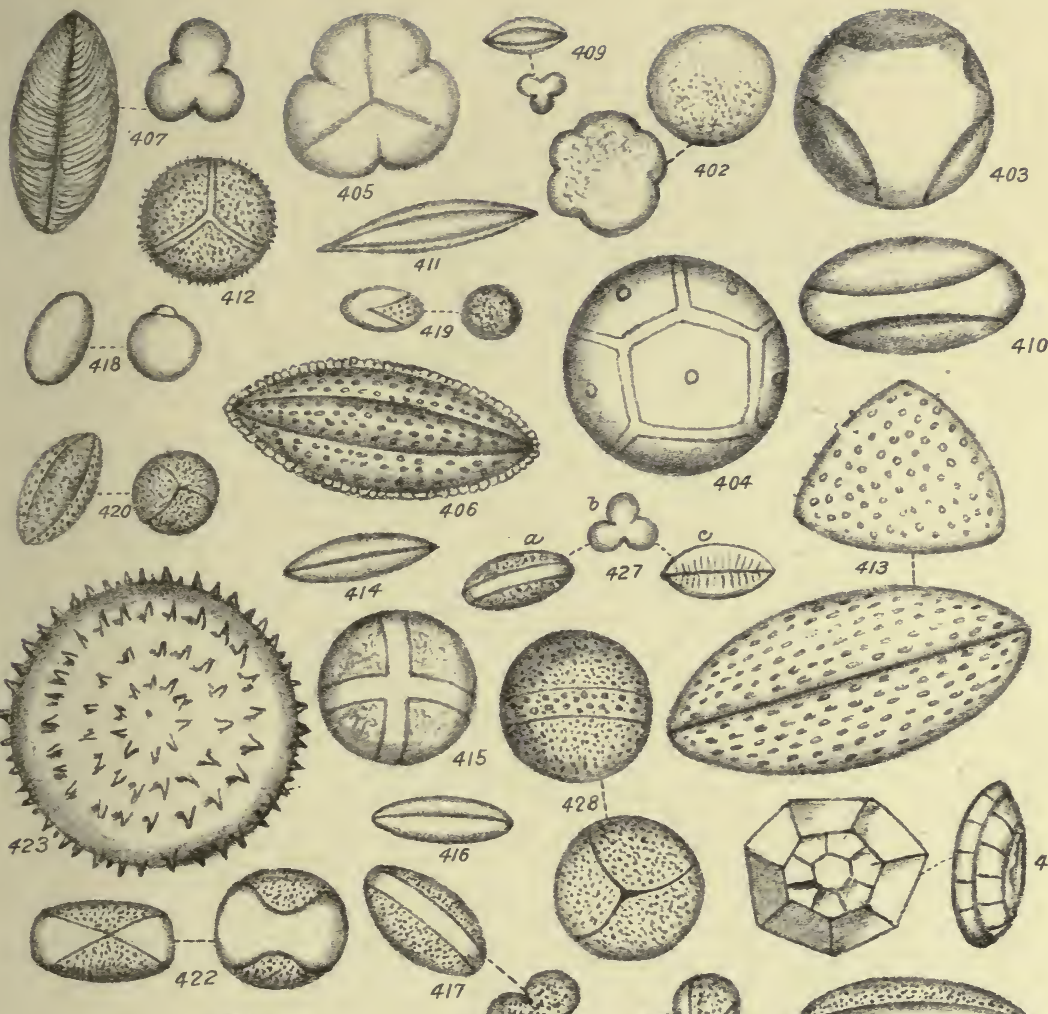

$\therefore$ (r) 10 3 427 4IIIIIIIII)
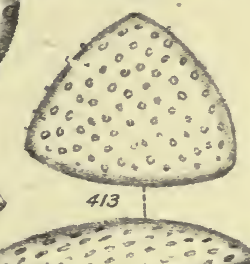


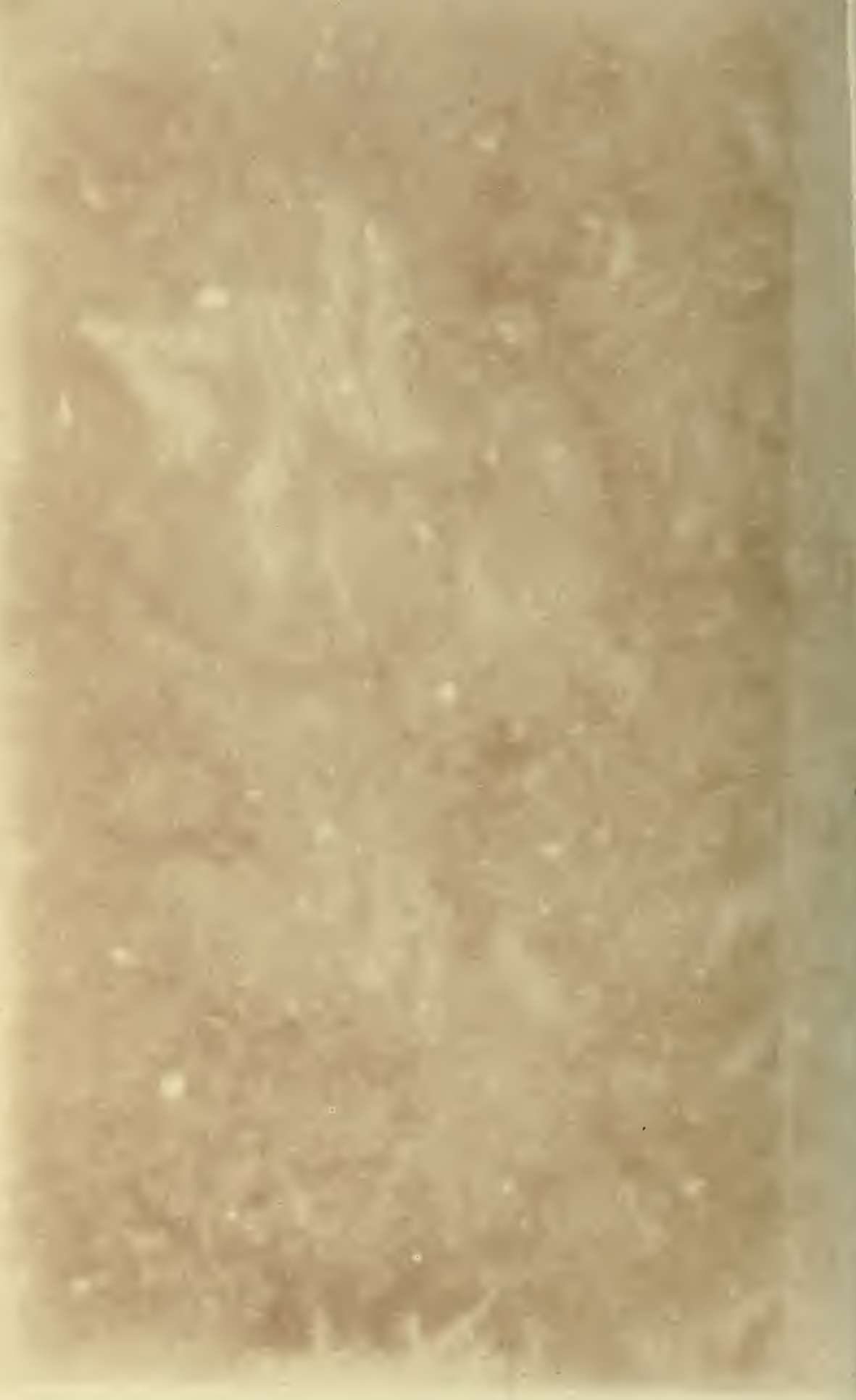



THIS BOOK IS DUE ON THE LAST DATE STAMPED BELOW

BOOKS REQUESTED BY ANOTHER BORROWER ARE SUBJECT TO RECALL AFTER ONE WEEK.

RENEWED BOOKS ARE SUBJECT TO IMMEDIATE RECALL

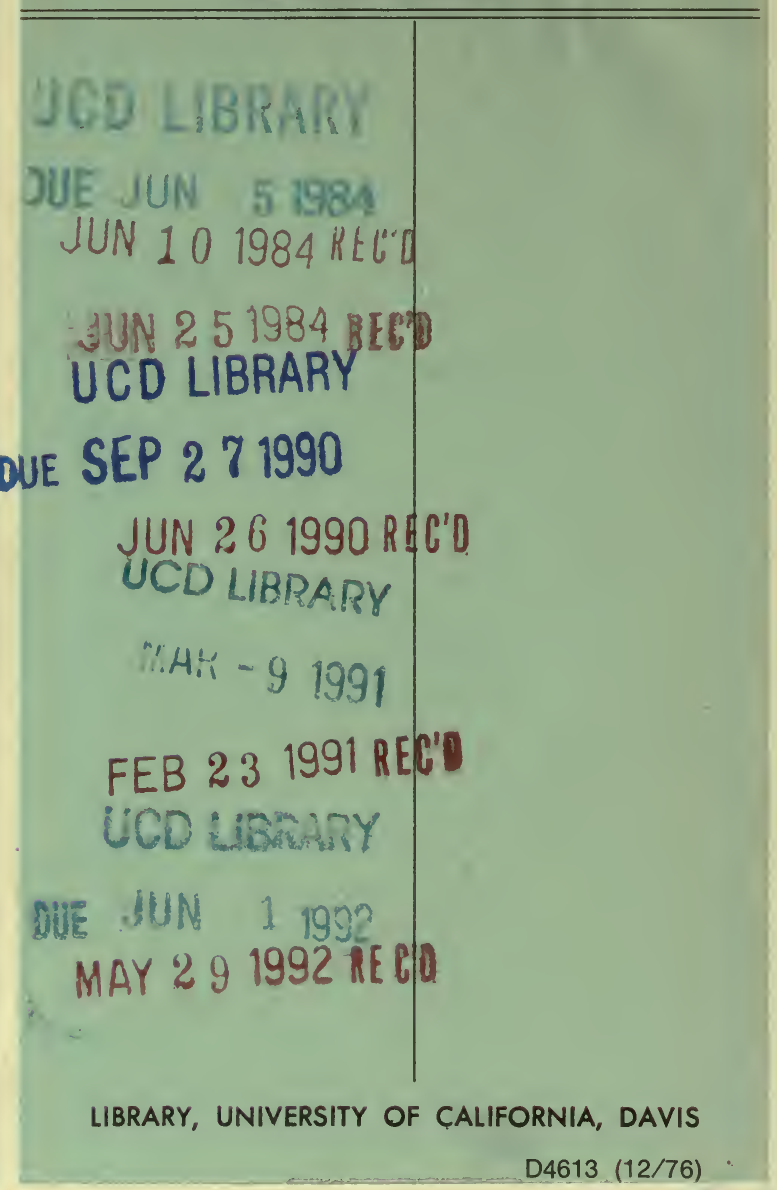




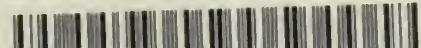 \\ 3 1175005405140}


